\title{
HIERARCHICALLY HYPERBOLIC SPACES I: CURVE COMPLEXES FOR CUBICAL GROUPS
}

\author{
JASON BEHRSTOCK, MARK F. HAGEN, AND ALESSANDRO SISTO
}

\begin{abstract}
In the context of CAT(0) cubical groups, we develop an analogue of the theory of curve complexes and subsurface projections. The role of the subsurfaces is played by a collection of convex subcomplexes called a factor system, and the role of the curve graph is played by the contact graph. There are a number of close parallels between the contact graph and the curve graph, including hyperbolicity, acylindricity of the action, the existence of hierarchy paths, and a Masur-Minsky-style distance formula.

We then define a hierarchically hyperbolic space; the class of such spaces includes a wide class of cubical groups (including all virtually compact special groups) as well as mapping class groups and Teichmüller space with any of the standard metrics. We deduce a number of results about these spaces, all of which are new for cubical or mapping class groups, and most of which are new for both. We show that the quasi-Lipschitz image from a ball in a nilpotent Lie group into a hierarchically hyperbolic space lies close to a product of hierarchy geodesics. We also prove a rank theorem for hierarchically hyperbolic spaces; this generalizes results of Behrstock-Minsky, Eskin-Masur-Rafi, Hamenstädt, and Kleiner. We finally prove that each hierarchically hyperbolic group admits an acylindrical action on a hyperbolic space. This acylindricity result is new for cubical groups, in which case the hyperbolic space admitting the action is the contact graph; in the case of the mapping class group, this provides a new proof of a theorem of Bowditch.
\end{abstract}

\section{Contents}

1. Introduction

2. Background

Part 1. Geometry of the contact graph 13

3. Hierarchy paths 13

4. Projection to the contact graph 14

5. Weak proper discontinuity of the action on the contact graph

6. Contractibility 16

7. Automorphisms of the contact graph 17

Part 2. Factor systems, projections, and the distance formula 18

8. Factored contact graphs 18

9. The distance formula

10. Projection of parallelism classes 28

11. Efficient embeddings into products of trees 30

12. Consistency and realization 34

Behrstock was supported as an Alfred P. Sloan Fellow, a Simons Fellowship, and by the National Science Foundation under Grant Number NSF 1006219.

Hagen was supported by the National Science Foundation under Grant Number NSF 1045119.

Sisto was supported by the Swiss National Science Foundation project 144373. 
Part 3. Hierarchically hyperbolic spaces 37

13. Quasi-boxes in hierarchically hyperbolic spaces 37

14. Acylindricity 44

References 48

\section{INTRODUCTION}

Cube complexes and groups that act on them are fundamental objects in geometric group theory. Examples of cubical groups - groups acting geometrically on CAT(0) cube complexes - are right-angled (and many other) Coxeter groups [Dav08, NR03, right-angled Artin groups [CD95], and, more generally, graph products of abelian groups [RW]. Other examples of cubical groups include: groups satisfying sufficiently strong small-cancellation conditions [Wis04]; many 3-manifold groups, including all hyperbolic ones [KM09, BW13, Wis] and some graph manifold groups [HP13]; hyperbolic free-by- $\mathbb{Z}$ groups [HW14]; etc. Despite the attention cubical groups have attracted in recent years, their large-scale geometry has remained rather opaque, with a few salient exceptions, notably the resolution of the Rank Rigidity Conjecture [CS11], characterizations of linear divergence, relative hyperbolicity, and thickness in terms of combinatorial data [BC12, Hag13, BH16], analysis of quasiflats in the 2 -dimensional case [BKS] and top-dimensional quasiflats in general [Hua].

Recently, there has been enormous progress in understanding the mapping class group and related spaces. Highlights have included resolutions of the Ending Lamination Conjecture [BCM12], the Rank Conjecture [BM08], quasi-isometric rigidity [BKMM12], finite asymptotic dimension [BBFb], and a number of others. Proofs of each of these results have featured the curve complex in a central position.

Motivated by the vital role the curve complex has played in unveiling the geometry of the mapping class group, in this work we develop analogues of those tools in the context of cubical groups. In particular, for cubical groups we develop versions of the machinery of curve complex projections and hierarchies initiated by Masur-Minsky in [MM99, MM00] as well as subsequent tools including the consistency and realization theorems [Beh06, BKMM12]. We note that right-angled Artin groups are a particularly interesting class of groups to which the tools we develop can be applied. Finally, we define hierarchically hyperbolic spaces, which provide a framework that encompasses mapping class groups, Teichmüller space, and a large class of cubical groups including the class of compact special groups of Haglund-Wise [HW08. This allows us to prove new results in both the mapping class group and cubical contexts.

1.1. Geometry of contact graphs. In Part 1, we develop a number of basic aspects of the geometry of contact graphs, extending a study which was initiated in Hag14. The contact graph $\mathcal{C} \mathcal{X}$ of the $\mathrm{CAT}(0)$ cube complex $\mathcal{X}$ is the intersection graph of the hyperplane carriers; in other words, there is a vertex for each hyperplane of $\mathcal{X}$, and two vertices are adjacent if the corresponding hyperplanes are not separated by a third. Since the contact graph is always hyperbolic (in fact, a quasi-tree) [Hag14], it is a natural candidate for a "curve complex" in the context of cubical groups. The main results of Part 1 are summarized below. Recall that the WPD property, as defined in [BF02], is a form of properness of an action "in the direction" of particular elements; see Section 5 for the precise definition. It has important applications to bounded cohomology (see e.g., $\mathrm{BBFa}$ ), is closely related to the Bestvina-Bromberg-Fujiwara construction [BBFb], and provides an equivalent characterization of acylindrical hyperbolicity Osi14 which is used in a number of applications.

Theorem A. Let $\mathcal{X}$ be a $C A T(0)$ cube complex and $\mathcal{C X}$ its contact graph. Then: 
(1) (WPD property.) If $\mathcal{X}$ is uniformly locally finite and $g \in \operatorname{Aut}(\mathcal{X})$ is rank-one with the property that no positive power of $g$ stabilizes a hyperplane, then $g$ acts as a loxodromic WPD element on $\mathcal{C X}$.

(2) (Hierarchy paths.) Let $x, y \in \mathcal{X}$ be 0 -cubes. Then there exist hyperplanes $H_{0}, \ldots, H_{k}$ with $x \in \mathcal{N}\left(H_{0}\right), y \in \mathcal{N}\left(H_{k}\right)$ and combinatorial geodesics $\gamma_{i} \rightarrow \mathcal{N}\left(H_{i}\right)$ such that $H_{0}, H_{1}, \ldots, H_{k}$ is a geodesic of $\mathcal{C X}$ and $\gamma_{0} \gamma_{1} \cdots \gamma_{k}$ is a geodesic joining $x, y$.

(3) (Contractibility.) If the set of 1 -cubes of $\mathcal{X}$ is countable and non-empty, then the flag complex spanned by $\mathcal{C X}$ is contractible.

In the case of mapping class groups, analogues of Theorem A. (1) and Theorem A. (2) were proved in [BF02] and [MM00]. The curve complex is not contractible, so Theorem A. (3) provides a way in which the contact graph is simpler than the curve complex.

Theorem A has applications to random walks. In particular, from Theorem A, (1), 2) and the main result of [MS], when the non-elementary group $G<\operatorname{Aut}(\mathcal{X})$ contains a rank-one element, random paths in $G$ stay close to geodesics with high probability. Further, this property has applications to various parameters associated with the random walk, including rate of escape and entropy.

1.2. Factor systems. The mapping class group, $\mathcal{M C G}(S)$, of a surface $S$ is associated with the curve complex of $S$, together with the collection of curve complexes of subsurfaces of $S$; this association underlies the hierarchy machinery of [MM00]. Analogously, a CAT(0) cube complex $\mathcal{X}$ contains a profusion of convex subcomplexes, each of which is itself a CAT $(0)$ cube complex, and the judicious choice of a family of convex subcomplexes enables the creation of hierarchy machinery. The role of the collection of subsurfaces is played by a factor system $\mathfrak{F}$ in $\mathcal{X}$, which is a particular uniformly locally finite collection of convex subcomplexes. Table 1 summarizes the analogy between the mapping class group and a CAT(0) cube complex with a geometric group action and a factor system. We emphasize that although the tools and the results we obtain have parallels for mapping class groups the techniques that we employ are very different.

\begin{tabular}{|c|c|}
\hline Mapping class group $\mathcal{M C G}(S)$ & Cube complex $\mathcal{X}$ with factor system $\mathfrak{F}$ and $G$-action \\
\hline Curve complex $\mathcal{C} S$ is hyperbolic MM99 & $\begin{array}{l}\text { Contact graph } \mathcal{C} \mathcal{X} \text { and factored contact graph } \hat{\mathcal{C}} \mathcal{X} \text { are hy- } \\
\text { perbolic, indeed, are quasi-trees (Thm. } 2.8 \text { Prop. } 8.25\end{array}$ \\
\hline $\mathcal{M C G}(S)$ acts on $\mathcal{C} S$ acylindrically Bow08 & $G$ acts on $\mathcal{C X}, \widehat{\mathcal{C}} \mathcal{X}$ acylindrically (Cor. 14.5 \\
\hline Nielsen-Thurston classification Thu88 & Loxodromic/reducible/generalized reducible (Thm. 2.9) \\
\hline $\begin{array}{l}\exists \text { quasi-geodesics in } \mathcal{M C G}(S) \text { shadowing geodesics } \\
\text { in } \mathcal{C} S \text { [MM00] }\end{array}$ & $\begin{array}{l}\exists \text { geodesics in } \mathcal{X} \text { shadowing geodesics in } \mathcal{C} \mathcal{X}, \mathcal{C} \mathcal{X} \\
\text { (Prop. 3.1. Prop. } 8.23\end{array}$ \\
\hline Subsurfaces & Subcomplexes in $\mathfrak{F}$ (Defn. 8.1 ) \\
\hline Projections to subsurfaces MM00 & Projection to $\hat{\mathcal{C}} F$ for $F \in \mathfrak{F}$ (Sec. 8.3 \\
\hline $\begin{array}{l}\text { Formula computing distance in } \mathcal{M C G}(S) \text { in terms } \\
\text { of curve complex distances [MM00] }\end{array}$ & $\begin{array}{l}\text { Formula computing distance in } \mathcal{X} \text { in terms of factored con- } \\
\text { tact graph distances (Thm. 9.1 }\end{array}$ \\
\hline Bounded Geodesic Image [MM00 & \begin{tabular}{|l|l|l} 
Bounded Geodesic Image, Prop. 8.20 & \\
\end{tabular} \\
\hline Nested, disjoint, overlapping subsurfaces & Parallel into, orthogonal, transverse elements of $\mathfrak{F}$ \\
\hline Large Link Lemma [MM00 & $\begin{array}{l}\text { Large Link Lemma, Prop. } \mid 9.4 \\
\end{array}$ \\
\hline Consistency and realization Beh06, BKMM12] & Consistency and realization (Thm. 12.4 ) \\
\hline
\end{tabular}

TABLE 1.

The collection of subcomplexes which constitute a factor system $\mathfrak{F}$ in $\mathcal{X}$ includes $\mathcal{X}$, as well as all combinatorial hyperplanes of $\mathcal{X}$, further, this collection is closed under the following operation: if $F, F^{\prime} \in \mathfrak{F}$ and $F$ has (combinatorial) projection onto $F^{\prime}$ of diameter more than some specified threshold, then the projection of $F$ onto $F^{\prime}$ lies in $\mathfrak{F}$. This implies that sufficiently large hyperplanes of any codimension belong to any factor system, and indeed 
each factor system $\mathfrak{F}$ contains a minimal factor system consisting of $\mathcal{X}$, all combinatorial hyperplanes, and the closure of this family under the above projection. This minimal factor system is $\operatorname{Aut}(\mathcal{X})$-invariant since each automorphism preserves the set of hyperplanes.

The reader should have in mind the following example, which already shows that the class of groups $G$ acting geometrically on cube complexes with $G$-invariant factor systems is very large. Let $\Gamma$ be a finite simplicial graph and let $\widetilde{S}_{\Gamma}$ be the universal cover of the Salvetti complex of the corresponding right-angled Artin group $A_{\Gamma}$, so that $\widetilde{S}_{\Gamma}$ is a $\operatorname{CAT}(0)$ cube complex on which $A_{\Gamma}$ acts properly with a single orbit of 0 -cubes [CD95]. Each induced subgraph $\Lambda$ of $\Gamma$ yields a monomorphism $A_{\Lambda} \rightarrow A_{\Gamma}$ and an $A_{\Lambda}$-equivariant embedding $\widetilde{S}_{\Lambda} \hookrightarrow \widetilde{S}_{\Gamma}$. The set of all such subcomplexes of $\widetilde{S}_{\Gamma}$, and all of their $A_{\Gamma}$-translates, forms a factor system for $\widetilde{S}_{\Gamma}$, described in detail in Section 8.2. This, and the fact that the existence of a factor system is inherited by convex subcomplexes (Lemma 8.5), enables the study of groups that are virtually special in the sense of Haglund-Wise [HW08] using factor systems:

Proposition B. Let $\overline{\mathcal{X}}$ be a special cube complex with finitely many hyperplanes. Then the universal cover $\mathcal{X}$ of $\overline{\mathcal{X}}$ contains a factor system, and hence contains a factor system that is invariant under the action of $\pi_{1} \overline{\mathcal{X}}$.

In Corollary 8.8, for special cube complexes, we describe the factor system explicitly in terms of the hyperplanes of $\overline{\mathcal{X}}$. Proposition $\mathrm{B}$ also enables one to study many cubical groups which are far from being special: in Section 11, using Proposition 8.3 together with BurgerMozes [BM00] and Wise [Wis07] we show there exists many non-virtually special groups $G$ which act geometrically on a $\mathrm{CAT}(0)$ cube complex $\mathcal{X}$ with a factor system. Moreover, we produce many examples which, unlike those of Burger-Mozes and Wise, do not admit equivariant embeddings into products of trees; these will be used in Section 11.

Each $F \in \mathfrak{F}$ is a convex subcomplex, and is thus a CAT(0) cube complex whose hyperplanes have the form $H \cap F$, where $H$ is a hyperplane of $\mathcal{X}$. This gives a natural injective graph homomorphism $\mathcal{C} F \rightarrow \mathcal{C X}$, whose image is an induced subgraph Hag14. Just as the elements of the factor system stand in analogy to the subsurfaces, the graphs $\mathcal{C} F$, where $F \in \mathfrak{F}$, essentially play the role of the curve complexes of the subsurfaces. In order to obtain Theorem 9.1 — our analogue of the Masur-Minsky distance formula — we must modify each $\mathcal{C} F$ slightly, by coning off each subgraph which is the contact graph of some smaller element of $\mathfrak{F}$. It is the resulting factored contact graphs $\widehat{\mathcal{C}} F$ that actually play the role of curve complexes. In Section 8.5, we show that factored contact graphs are all quasi-trees. Moreover, when $\mathfrak{F}$ is the minimal factor system described above, then $\hat{\mathcal{C}} \mathcal{X}$ and $\mathcal{C} \mathcal{X}$ are quasi-isometric.

In Section 9, we prove the following analogue for cubical groups of the celebrated MasurMinsky distance formula [MM00, Theorem 6.12]. Their formula has become an essential tool in studying the geometry of the mapping class group. Later, we will take the existence of such a formula as one of the characteristic features of a hierarchically hyperbolic space.

Theorem $\mathbf{C}$ (Distance formula). Let $\mathcal{X}$ be a $C A T(0)$ cube complex with a factor system $\mathfrak{F}$. Let $\overline{\mathfrak{F}}$ contain exactly one representative of each parallelism class in $\mathfrak{F}$. Then there exists $s_{0} \geqslant 0$ such that for all $s \geqslant s_{0}$, there are constants $K \geqslant 1, C \geqslant 0$ such that for all $x, y \in \mathcal{X}^{(0)}$,

$$
\mathrm{d}_{\mathcal{X}}(x, y) \asymp_{K, C} \sum_{F \in \overline{\mathfrak{F}}}\left\{\left\{\mathrm{d}_{\widehat{\mathcal{C}} F}\left(\pi_{F}(x), \pi_{F}(y)\right)\right\}_{s} .\right.
$$

(Here, $\{A\}_{s}=A$ if $A \geqslant s$ and 0 otherwise. The notation $\asymp_{K, C}$ means "up to bounded multiplicative and additive error".)

In Theorem C, we use the notion of parallelism: two convex subcomplexes $F, F^{\prime}$ of $\mathcal{X}$ are parallel if for all hyperplanes $H$, we have $H \cap F \neq \varnothing$ if and only if $H \cap F^{\prime} \neq \varnothing$. Equivalently, $F, F^{\prime}$ are parallel if and only if $\mathcal{C} F, \mathcal{C} F^{\prime}$ are the same subgraph of $\mathcal{C} \mathcal{X}$; parallel subcomplexes 
are isomorphic. Just as the Masur-Minsky distance formula involves summing over all curve complexes of subsurfaces, by identifying parallel elements of the factor system, our sum is over all factored contact graphs without repetition.

Another important property of the curve complex $\mathcal{C} S$ is that the action of $\mathcal{M C G}(S)$ on $\mathcal{C} S$ is acylindrical, by a result of Bowditch Bow08. We obtain an analogous result for actions on (factored) contact graphs arising from actions on cube complexes, and, in Section 14 we will show this holds in considerably greater generality . The statement in the cubical case is:

Theorem D (Acylindrical hyperbolicity from factor systems). Let the group $G$ act properly and cocompactly on the $C A T(0)$ cube complex $\mathcal{X}$ and suppose that $\mathcal{X}$ contains a factor system. Then the induced action of $G$ on the contact graph $\mathcal{C} \mathcal{X}$ of $\mathcal{X}$ is acylindrical.

Theorem D and the results of [MT14] combine to yield the following, which is related to work of Nevo-Sageev on Poisson boundaries of cube complexes [NS13]:

Corollary E (Poisson boundary). Let the group $G$ act properly and cocompactly on the $C A T(0)$ cube complex $\mathcal{X}$ and suppose that $\mathcal{X}$ contains a factor system. Let $\mu$ be a probability distribution on $G$ with finite entropy whose support generates a non-elementary group acting on $\mathcal{C X}$ and let $\nu$ be the hitting measure on $\partial \mathcal{C X}$. Then $(\partial \mathcal{C X}, \nu)$ is isomorphic to the Poisson boundary of $(G, \mu)$.

Theorem $\mathrm{D}$ also allows one to produce free subgroups of $G$ freely generated by finite collections of high powers of elements, each of which acts loxodromically on $\mathcal{C X}$ (Corollary 14.6).

Using Theorem C, together with the tools in [BBFb], the fact that factored contact graphs are quasi-trees, and the machinery we develop in Section 10, we prove:

Theorem F. Let $G$ act properly and cocompactly on the $C A T(0)$ cube complex $\mathcal{X}$ and suppose that $\mathcal{X}$ contains a $G$-invariant factor system. If the action of $G$ is hereditarily flip-free, then $G$ quasi-isometrically embeds in the product of finitely many quasi-trees.

Moreover, there exist such $G, \mathcal{X}$ such that, for all finite-index subgroups $G^{\prime} \leqslant G$, there is no $G^{\prime}$-equivariant isometric embedding of $\mathcal{X}$ in the product of finitely many simplicial trees.

Hereditary flip-freeness is a mild technical condition on the factor system $\mathfrak{F}$ which holds for most of the examples we have discussed. For the second assertion of the theorem, we exploit the existence of cocompactly cubulated groups with no finite quotients [BM00, Wis07]. These groups are lattices in products of trees stabilizing the factors; this property gives rise to a factor system. The space for this example is assembled from these pieces in such a way that the existence of a factor system persists, but there is no longer a finite equivariant coloring of the hyperplanes with intersecting hyperplanes colored differently. This lack of a coloring precludes the existence of an isometric embedding in a product of finitely many trees.

1.3. Comparison to the theory of the extension graph of a right-angled Artin group. In the special case where $\mathcal{X}=\widetilde{S}_{\Gamma}$ is the universal cover of the Salvetti complex $S_{\Gamma}$ of a right-angled Artin group $A_{\Gamma}$, the machinery of factor systems and contact graphs is not the first attempt to define an analogue of the curve complex and the attendant techniques. In KK13, Kim-Koberda introduced the extension graph $\Gamma^{e}$ associated to the finite simplicial graph $\Gamma$ (and thus to $A_{\Gamma}$ ). This graph has a vertex for each conjugate of each standard generator of $A_{\Gamma}$ (i.e., vertex of $\Gamma$ ), with adjacency recording commutation. In the same paper it is shown that, like $\mathcal{C} \widetilde{S}_{\Gamma}$, the extension graph is always quasi-isometric to a tree, and in [KK14], the analogy between $\mathcal{M C G}(S)$, with its action on $\mathcal{C} S$, and $A_{\Gamma}$, with its action on $\Gamma^{e}$, is extensively developed: it is shown, for instance, that this action is acylindrical and obeys a loxodromic-elliptic dichotomy. It is observed in [KK14] that, except in exceptional cases, there is a surjective graph homomorphism $\mathcal{C} \widetilde{S}_{\Gamma} \rightarrow \Gamma^{e}$, where $\widetilde{S}_{\Gamma}$ is the universal cover 
of the Salvetti complex of $A_{\Gamma}$, which is also a quasi-isometry, so many such geometric results about the action of $A_{\Gamma}$ on $\Gamma^{e}$ can be deduced from the results of the present paper about the action of $A_{\Gamma}$ on $\mathcal{C} \widetilde{S}_{\Gamma}$. It should be strongly emphasized that the papers [KK14, KK13] also explore interesting and less purely geometric issues, particular to right-angled Artin groups, that cannot be treated with factor system tools.

The authors of [KK14] also set up some version of hierarchy machinery, with the role of subsurfaces being played by subgroups of $A_{\Gamma}$ of the form $A_{\mathrm{Lk}(v)}$, where $v$ is a vertex of $\Gamma$, and their conjugates. When $\Gamma$ has girth at least 5, they obtain a distance formula KK14, Proposition 65], but, as they note, the formula they give has significant differences with the Masur-Minsky distance formula for the mapping class group. For example, the sum is taken over specified projections, which depend on the points whose distance is being estimated, rather than over all projections. Another significant distinction is that their distance formula does not measure distance in the right-angled Artin group $A_{\Gamma}$, but rather it measures the syllable length in that space (although not a perfect analogy: their metric is more similar to the Weil-Petersson metric on Teichmüller space than to the word metric on the mapping class group). The extension graph seems unable to capture distance in the right-angled Artin group via a hierarchical construction, since the extension graph is bounded when $A_{\Gamma}=\mathbb{Z}$. In the present paper, the geometric viewpoint afforded by factor systems, and in particular the existence of hierarchy paths (Proposition 8.23) and a Large Link Lemma (Proposition 8.20) allows us to overcome these issues.

1.4. Hierarchically hyperbolic spaces. Our aim in the last part of the paper is to develop a unified framework to study mapping class group and CAT(0) cube complexes from a common perspective. To this end, we axiomatize the machinery of factored contact graphs/curve complexes, distance formula, etc., to obtain the definition of a hierarchically hyperbolic space, which is formally stated in Definition 13.1. This notion includes the two classes of groups just mentioned and allows one to prove new results for both of these classes simultaneously. Hierarchically hyperbolic spaces come with a notion of complexity: complexity 0 corresponds to bounded spaces, infinite diameter $\delta$-hyperbolic spaces have complexity 1 , and higher complexity hierarchically hyperbolic spaces coarsely contain direct products.

Roughly, a space $\mathcal{X}$ is hierarchically hyperbolic if $\mathcal{X}$ can be equipped with a set $\mathfrak{S}$ of uniformly Gromov-hyperbolic spaces, and projections $\mathcal{X} \rightarrow W$, with $W \in \mathfrak{S}$. These projections are required to satisfy various properties reminiscent of those satisfied by subsurface projections in the mapping class group case and projections to factored contact graphs in the case of $\mathrm{CAT}(0)$ cube complexes with factor systems. Hence a space $\mathcal{X}$ may be hierarchically hyperbolic in multiple ways, i.e., with respect to projections to distinct families of hyperbolic spaces.

Remark (HHS is a QI-invariant property). It is easily seen from Definition 13.1 that, if $\mathcal{X}$ is hierarchically hyperbolic by virtue of its projections to a set $\mathfrak{S}$ of hyperbolic spaces, and $\mathcal{Y} \rightarrow \mathcal{X}$ is a quasi-isometry, then we can compose each projection with the quasi-isometry and conclude that $\mathcal{Y}$ is hierarchically hyperbolic with respect to the same set $\mathfrak{S}$.

The motivating examples of hierarchically hyperbolic spaces are as follows:

Theorem G (Hierarchically hyperbolic spaces).

(1) A CAT(0) cube complex with a factor system $\mathfrak{F}$ is hierarchically hyperbolic with respect to the set of factored contact graphs $\hat{\mathcal{C}} W$, with $W \in \mathfrak{F}$. (This is summarized in Remark 13.2.)

(2) Let $S$ be a connected, oriented hyperbolic surface of finite type. Then $\mathcal{M C G}(S)$ is hierarchically hyperbolic with respect to the collection of curve complexes of subsurfaces of $S$ [MM99, MM00, Beh06, BKMM12]. 
(3) Teichmüller space $\mathcal{T}(S)$ with the Weil-Petersson metric is hierarchically hyperbolic with respect to curve complexes of non-annular subsurfaces of $S$ MM99, MM00, Bro03, Beh06, BKMM12.

(4) $\mathcal{T}(S)$ with the Teichmüller metric is hierarchically hyperbolic with respect to curve complexes of non-annular subsurfaces and combinatorial horoballs associated to annuli [MM99, Raf07, Dur, EMR].

In a forthcoming paper we will show that fundamental groups of non-geometric 3-manifolds are also hierarchically hyperbolic, and that a metric space that is hyperbolic relative to a collection of hierarchically hyperbolic subspaces is hierarchically hyperbolic [BHS15]. We note that it is already known that relatively hyperbolic groups admit a distance formula Sis13. Another interesting question is whether a right-angled Artin group endowed with the syllable length metric is a hierarchically hyperbolic space.

In Section 13, after defining hierarchically hyperbolic spaces, we study quasi-Lipschitz maps from balls in $\mathbb{R}^{n}$, and more general nilpotent Lie groups, into these spaces. The next three results will all follow directly from our Theorem 13.11 which provides a single unifying statement in terms of asymptotic cones. Our first result is a generalization of a result from [EMR, Theorem A] which is about mapping class groups and Teichmüller spaces.

Theorem $\mathbf{H}$ (Quasi-boxes in hierarchically hyperbolic spaces). Let $\mathcal{X}$ be a hierarchically hyperbolic space. Then for every $n \in \mathbb{N}$ and every $K, C, R_{0}, \epsilon_{0}$ the following holds. There exists $R_{1}$ so that for any ball $B \subseteq \mathbb{R}^{n}$ of radius at least $R_{1}$ and $f: B \rightarrow \mathcal{X}$ a $(K, C)$-quasiLipschitz map, there is a ball $B^{\prime} \subseteq B$ of radius $R^{\prime} \geqslant R_{0}$ such that $f\left(B^{\prime}\right)$ lies inside the $\epsilon_{0} R^{\prime}-$ neighborhood of a standard box.

In Theorem $\mathrm{H}$, we do not require $B, B^{\prime}$ to be centered at the same point in $\mathbb{R}^{n}$.

Our proof uses methods different from those used in EMR. Our approach is much shorter and does not rely on partitions of the set of subsurfaces (or an analogue thereof), which plays an important role in their proof. In particular, we do not rely on the results from $[\mathrm{BBFb}$; this is one reason why our results can be applied in the case of CAT(0) cube complexes where the techniques of [EMR would fail. However, our approach and theirs share some commonalities, for instance, we use Rademacher's Theorem (applied to maps that arise at the level of asymptotic cones), while in [EMR], the authors use a coarse differentiation result.

Using a generalization of Rademacher's theorem due to Pansu, we consider the case of quasi-Lipschitz maps from more general nilpotent Lie groups.

Theorem I (Restriction on nilpotent groups in hierarchically hyperbolic spaces). Let $\mathcal{X}$ be a hierarchically hyperbolic space. Then for every simply connected nilpotent Lie group $\mathcal{N}$, with a left-invariant Riemannian metric, and every $K, C$ there exists $R$ with the following property. For every $(K, C)$-quasi-Lipschitz map $f: B \rightarrow \mathcal{X}$ from a ball in $\mathcal{N}$ into $\mathcal{X}$ and for every $n \in \mathcal{N}$ we have $\operatorname{diam}(f(B \cap n[\mathcal{N}, \mathcal{N}])) \leqslant R$. In particular, if a finitely generated nilpotent group admits a quasi-isometric embedding into $\mathcal{X}$ then it is virtually abelian.

The final conclusion of Theorem I is known in the case where $\mathcal{X}$ is a CAT(0) space Pau01. Although it does not appear to be in the literature, the conclusion of Theorem $\mathbb{I}$ for $\mathcal{M C G}$ can be alternatively proved using [Hum] and the results of [Pau01].

The following theorem generalizes the Rank Theorems from [BM08, EMR, Ham, Kle99]:

Theorem $\mathbf{J}$ (Rank). Let $\mathcal{X}$ be a hierarchically hyperbolic space with respect to a set $\mathfrak{S}$. If there exists a quasi-isometric embedding $\mathbb{R}^{n} \rightarrow \mathcal{X}$ then $n$ is at most the maximal cardinality of a set of pairwise-orthogonal elements of $\mathfrak{S}$ and, in particular, at most the complexity of $\mathcal{X}$. 
When $\mathcal{X}$ is a $\operatorname{CAT}(0)$ cube complex with a factor system and $\operatorname{Aut}(\mathcal{X})$ acts cocompactly, then such a space naturally has two hierarchically hyperbolic structures. One of these structures has hierarchy paths that are combinatorial geodesics and one with $\mathrm{CAT}(0)$ geodesics; the first is obtained explicitly in Section 8.3 and the existence of the latter follows from the first via a simple argument about projections of CAT(0) geodesics and convex hulls of $\ell_{1}$ geodesics to factored contact graphs. By Theorem $\mathrm{H}$ and cocompactness, the existence of a quasi-isometric embedding $\mathbb{R}^{n} \rightarrow \mathcal{X}$ then implies that $\mathcal{X}$ contains both an $\ell_{1}$-isometrically embedded copy of $\mathbb{R}^{n}$ with the standard tiling and, in the CAT(0) metric, an isometrically embedded flat of dimension $n$. We thus recover the cubical version of a theorem of Kleiner, see [Kle99, Theorem C]. We also note that, in the special case of top-dimensional quasiflats in CAT(0) cube complexes, Huang has very recently proved a stronger statement [Hua, Theorem 1.1].

Finally, we relate hierarchically hyperbolic groups to acylindrically hyperbolic groups, as studied in Osi14. Although natural, the definition of an automorphism of a hierarchically hyperbolic space is technical, but includes, in the relevant cases, all elements of $\mathcal{M C G}$ and all isometries of a cube complex with a factor system. The "maximal element" $S$ and $\hat{\mathcal{C}} S$ referred to below are $\mathcal{X}$ and its factored contact graph in case $\mathcal{X}$ is a CAT(0) cube complex, while they are the surface $S$ and its curve complex when $G$ is the mapping class group of $S$.

Theorem K. Let $\mathcal{X}$ be hierarchically hyperbolic with respect to the set $\mathfrak{S}$ of hyperbolic spaces and let $G \leqslant \operatorname{Aut}(\mathfrak{S})$ act properly and cocompactly on $\mathcal{X}$. Let $S$ be the maximal element of $\mathfrak{S}$ and denote by $\hat{\mathcal{C}} S$ the corresponding hyperbolic space. Then $G$ acts acylindrically on $\hat{\mathcal{C}} S$.

In the case of the acylindricity of the action of $\mathcal{M C G}$ on the curve complex, our argument provides a new proof of a result of Bowditch [Bow08]. We note that our proof is substantially different and is also rather short (although it does relies on some amount of machinery).

Throughout the section on hierarchically hyperbolic spaces, we make use of a notion of "gate," which, in addition to the uses in this paper, we believe will be very useful for other applications as well. This notion simultaneously generalizes gates/projections on (certain) convex subspaces in the cubical context and coarsely Lipschitz retractions of the marking complex on its natural subspaces associated with subsurfaces. Nonetheless, the definition we give exploits a different point of view, which turns out to be very convenient and allows us to give different (and concise) proofs than previously existing ones for a number of results about mapping class groups and CAT(0) cube complexes.

Acknowledgements. The authors thank Matthew Durham, Jingyin Huang, Sang-hyun Kim, Bruce Kleiner, Thomas Koberda, Urs Lang, and Kasra Rafi for stimulating discussions and Chris O'Donnell for a helpful correction. We also thank those that have given us helpful feedback on this paper, especially Jacob Russell and the anonymous referees for their numerous useful comments.

\section{BACKGROUND}

We assume that the reader is familiar with basic properties of $\mathrm{CAT}(0)$ cube complexes, hyperplanes, median graphs, etc., and refer the reader to e.g., [BC08, CN05, Che00, Hag14, Hag13, Hag08, Wis, Wis11 for background on these concepts as they are used in this paper. Nonetheless, in Section 2.1 and Section 2.2, we will review cubical convexity and make explicit the notions of gate, projection, and parallelism, since they play a fundamental role, and recall several useful facts about the contact graph. In Section 2.3 , we briefly discuss background on right-angled Artin groups. Since, in Section 13, we will use asymptotic cones, we refer the reader to [Dru02] for the relevant background. 
2.1. Convex subcomplexes, combinatorial hyperplanes, gates, and parallelism. Throughout, $\mathcal{X}$ is a $\mathrm{CAT}(0)$ cube complex and $\mathcal{H}$ is the set of hyperplanes. Unless stated otherwise, we work in the 1 -skeleton of $\mathcal{X}$, and we denote by $\mathrm{d}_{\mathcal{X}}$ the graph metric on $\mathcal{X}^{(1)}$. The contact graph $\mathcal{C X}$, defined in [Hag14], is the graph whose vertex set is $\mathcal{H}$, with two vertices adjacent if the corresponding hyperplanes are not separated by a third.

The subcomplex $K \subset \mathcal{X}$ is full if $K$ contains each cube of $\mathcal{X}$ whose 1 -skeleton appears in $K$. A subcomplex $K \subset \mathcal{X}$ is isometrically embedded if $K$ is full and $K \cap \bigcap_{i} H_{i}$ is connected for all $\left\{H_{i}\right\} \subset \mathcal{H}$. In this case, we say that $H \in \mathcal{H}$ crosses $K$ when $K \cap H \neq \varnothing$. The term "isometrically embedded" is justified by the well-known fact (see e.g., [Hag14]) that, if $K \subset \mathcal{X}$ is isometrically embedded in this sense, then $K^{(1)} \hookrightarrow \mathcal{X}^{(1)}$ is an isometric embedding with respect to graph metrics. The isometrically embedded subcomplex $K \subset \mathcal{X}$ is convex if any of the following equivalent conditions is met:

(1) $K$ coincides with the intersection of all combinatorial halfspaces (see below) containing $K$.

(2) Let $x, y, z \in \mathcal{X}$ be 0 -cubes with $x, y \in K$. Then the median of $x, y, z$ lies in $K$.

(3) $K^{(1)}$ contains every geodesic of $\mathcal{X}^{(1)}$ whose endpoints lie in $K^{(1)}$.

(4) Let $c$ be an $n$-cube of $\mathcal{X}$, with $n \geqslant 2$. Suppose that $c$ has $n$ codimension- 1 faces that lie in $K$. Then $c \subset K$.

(5) The inclusion $K \rightarrow \mathcal{X}$ is a local isometry.

(Recall that a combinatorial map $\phi: K \rightarrow \mathcal{X}$ of cube complexes, with $\mathcal{X}$ nonpositivelycurved, is a local isometry if $\phi$ is locally injective and, for each $x \in K^{(0)}$, the map induced by $\phi$ on the link of $x$ is injective and has image a full subcomplex of the link of $\phi(x)$; see [HW08, Wis, Wis11] for more on local isometries and local convexity.)

A convex subcomplex $K \subseteq \mathcal{X}$ is itself a CAT(0) cube complex, whose hyperplanes are the subspaces of the form $H \cap K$, where $H \in \mathcal{H}$. A useful mantra, following from the definition of convexity, is: "if $K$ is convex, then any two hyperplanes that cross $K$ and cross each other must cross each other inside of $K$ ". The convex hull of $Y \subseteq \mathcal{X}$ is the intersection of all convex subcomplexes containing $Y$.

It follows immediately from the definition that if $K \subseteq \mathcal{X}$ is convex, then for all $x \in \mathcal{X}^{(0)}$, there exists a unique closest 0 -cube $\mathfrak{g}_{K}(x)$, called the gate of $x$ in $K$. The gate is characterized by the property that $H \in \mathcal{H}$ separates $\mathfrak{g}_{K}(x)$ from $x$ if and only if $H$ separates $x$ from $K$.

As discussed in [Hag14, it follows from the definition of convexity that the inclusion $K \hookrightarrow \mathcal{X}$ induces an injective graph homomorphism $\mathcal{C} K \rightarrow \mathcal{C} \mathcal{X}$ whose image is a full subgraph: just send each $H \cap K$ to $H$. This allows us to define a projection $\mathfrak{g}_{K}: \mathcal{X} \rightarrow K$. The map $x \mapsto \mathfrak{g}_{K}(x)$ extends to a cubical map $\mathfrak{g}_{K}: \mathcal{X} \rightarrow K$ as follows. Let $c$ be a cube of $\mathcal{X}$ and let $H_{1}, \ldots, H_{d}$ be the collection of pairwise-crossing hyperplanes crossing $c$. Suppose that these are labeled so that $H_{1}, \ldots, H_{s}$ cross $K$, for some $0 \leqslant s \leqslant d$, and suppose that $H_{s+1}, \ldots, H_{d}$ do not cross $K$. Then the 0 -cubes of $c$ map by $g_{K}$ to the 0 -cubes of a uniquely determined cube $\mathfrak{g}_{K}(c)$ of $K$ in which the hyperplanes $H_{1}, \ldots, H_{s}$ intersect, and there is a cubical collapsing map $c \cong[-1,1]^{d} \rightarrow[-1,1]^{s} \cong \mathfrak{g}_{K}(c)$ extending the gate map on the 0 -skeleton. This map is easily seen to be compatible with the face relation for cubes, yielding the desired cubical map $\mathfrak{g}_{K}: \mathcal{X} \rightarrow K$, whose salient property is that for all $x \in \mathcal{X}$, a hyperplane $H$ separates $x$ from $K$ if and only if $H$ separates $x$ from $\mathfrak{g}_{K}(x)$. The next lemma follows easily from the definitions and is used freely throughout this paper:

Lemma 2.1. Let $\mathcal{X}$ be a $C A T(0)$ cube complex, let $A \subseteq B \subseteq \mathcal{X}$ be convex subcomplexes, and let $x, y \in \mathcal{X}$ be 0 -cubes. Then any hyperplane separating $\mathfrak{g}_{A}(x), \mathfrak{g}_{A}(y)$ separates $\mathfrak{g}_{B}(x), \mathfrak{g}_{B}(y)$ (and hence separates $x, y$ ). 
Definition 2.2 (Parallel). Convex subcomplexes $F, F^{\prime} \subseteq \mathcal{X}$ are parallel if for each hyperplane $H$, we have $H \cap F \neq \varnothing$ if and only if $H \cap F^{\prime} \neq \varnothing$. Parallelism is clearly an equivalence relation.

Hyperplanes lead to important examples of parallel subcomplexes. For each hyperplane $H \in \mathcal{H}$, let $\mathcal{N}(H)$ denote its carrier, i.e., the union of all closed cubes intersecting $H$. Then there is a cubical isometric embedding $H \times[-1,1] \cong \mathcal{N}(H) \hookrightarrow \mathcal{X}$, and we denote by $H^{ \pm}$be the images of $H \times\{ \pm 1\}$. These convex subcomplexes are combinatorial hyperplanes. Observe that $H^{+}$and $H^{-}$are parallel: a hyperplane crosses $H^{ \pm}$if and only if it crosses $H$. (Very occasionally, it will be convenient to refer to combinatorial halfspaces - a combinatorial halfspace associated to $H \in \mathcal{H}$ is a component of $\mathcal{X}-H \times(-1,1)$. A component of the boundary of such a combinatorial halfspace is one of $H^{+}$or $H^{-}$.)

Remark 2.3 (Parallelism and dual cube complexes). The reader accustomed to thinking of cube complexes using Sageev's construction of the cube complex dual to a space with walls Sag95, CN05] might appreciate the following characterization of parallelism: the convex subcomplex $F$ can be viewed as the dual to a wallspace whose underlying set is $\mathcal{X}^{(0)}$ and whose walls are the hyperplanes in the vertex set of $\mathcal{C} F$ - one must check that in this case, the restriction quotient $\mathcal{X} \rightarrow F$ is split by a cubical isometric embedding $F \rightarrow \mathcal{X}$. In general, the splitting need not be unique, depending on a choice of basepoint, and the images of the various embeddings are exactly the representatives of the parallelism class of $F$.

Observe that $F, F^{\prime}$ are parallel if and only if their contact graphs are the same subgraph of $\mathcal{C X}$. Parallel subcomplexes are isomorphic and in fact, the following stronger statement holds, and we shall use it throughout the paper.

Lemma 2.4. Let $F, F^{\prime} \subseteq \mathcal{X}$ be convex subcomplexes. The following are equivalent:

(1) $F$ and $F^{\prime}$ are parallel;

(2) there is a cubical isometric embedding $F \times[0, a] \rightarrow \mathcal{X}$ whose restrictions to $F \times$ $\{0\}, F \times\{a\}$ factor as $F \times\{0\} \cong F \hookrightarrow \mathcal{X}$ and $F \times\{a\} \cong F^{\prime} \hookrightarrow \mathcal{X}$, and $[0, a]$ is a combinatorial geodesic segment crossing exactly those hyperplanes that separate $F$ from $F^{\prime}$.

Hence there exists a convex subcomplex $E_{F}$ such that there is a cubical embedding $F \times E_{F}$ with convex image such that for each $F^{\prime}$ in the parallelism class of $F$, there exists a 0 -cube $e \in E_{F}$ such that $F \times\{e\} \rightarrow \mathcal{X}$ factors as $F \times\{e\} \stackrel{i d}{\rightarrow} F^{\prime} \hookrightarrow F$.

Proof. Let $F, F^{\prime}$ be parallel, let $\mathcal{H}(F)$ be the set of hyperplanes crossing $F$ (hence $\mathcal{H}\left(F^{\prime}\right)=$ $\mathcal{H}(F))$ and let $\mathcal{S}$ be the finite set of hyperplanes separating $F$ from $F^{\prime}$. Let $E$ be the cubical convex hull of $F \cup F^{\prime}$. Then every element of $\mathcal{H}(F)$ crosses $E$, and the same is true of $\mathcal{S}$, since any geodesic starting on $F$ and ending on $F^{\prime}$ must contain a 1-cube dual to each element of $\mathcal{S}$. Conversely, let $H \in \mathcal{H}$ cross the hull of $F \cup F^{\prime}$ and suppose that $H \notin \mathcal{H}(F) \cup \mathcal{S}$. Then either $H$ crosses $F$ but not $F^{\prime}$, which is impossible, or $F$ and $F^{\prime}$ lie in the same halfspace $\overleftarrow{H}$ associated to $H$. But then $\overleftarrow{H}$ contains a combinatorial halfspace $\overleftarrow{H}^{*}$ that contains $F$, $F^{\prime}$, so $E \cap \overleftarrow{H}^{*}$ is a convex subcomplex containing $F \cup F^{\prime}$ and properly contained in $E$ (since $H$ does not cross $E \cap \overleftarrow{H}^{*}$ ). This contradicts that $E$ is the convex hull. Hence the set of hyperplanes crossing $E$ is precisely $\mathcal{H}(F) \cup \mathcal{S}$. Each hyperplane in $\mathcal{H}(F)$ crosses each hyperplane in $\mathcal{S}$, and it follows from [CS11, Proposition 2.5] that $E \cong F \times I$ for some convex subcomplex $I$ such that the set of hyperplanes crossing $I$ is precisely $\mathcal{S}$. It is easily verified that $I$ is the convex hull of a geodesic segment. This proves $(1) \Rightarrow(2)$; the other direction is obvious.

The "hence" assertion follows from an identical argument, once $\mathcal{S}$ is taken to be the set of hyperplanes $H$ with the property that for some $F^{\prime}, F^{\prime \prime}$ in the parallelism class of $F$, we have that $H$ separates $F$ from $F^{\prime}$. 
Lemma 2.5. For each convex subcomplex $F \subseteq \mathcal{X}$, either $F$ is unique in its parallelism class, or $F$ is contained in a combinatorial hyperplane.

Proof. If $F$ is parallel to some $F^{\prime} \neq F$, then as discussed above, there is a cubical isometric embedding $F \times[0, a] \rightarrow \mathcal{X}$, with $a \geqslant 1$, whose restriction to $F \times\{0\}$ is the inclusion $F \hookrightarrow \mathcal{X}$. Cubical isometric embeddings take combinatorial hyperplanes to subcomplexes of combinatorial hyperplanes, and $F \times\{0\}$ is a combinatorial hyperplane of $F \times[0, a]$ since $a \geqslant 1$, so the claim follows.

The following will be used often starting in Section 8.1.

Lemma 2.6. If $F, F^{\prime}$ are convex subcomplexes, then $\mathfrak{g}_{F}\left(F^{\prime}\right)$ and $\mathfrak{g}_{F^{\prime}}(F)$ are parallel subcomplexes. Moreover, if $F \cap F^{\prime} \neq \varnothing$, then $\mathfrak{g}_{F}\left(F^{\prime}\right)=\mathfrak{g}_{F^{\prime}}(F)=F \cap F^{\prime}$;

Proof. Suppose that $H$ is a hyperplane crossing $\mathfrak{g}_{F}\left(F^{\prime}\right)$. Then $H$ separates $\mathfrak{g}_{F}(x), \mathfrak{g}_{F}(y)$ for some $x, y \in F^{\prime}$. By Lemma 2.1, $H$ separates $x, y$ and hence crosses $F^{\prime}$. Thus $H$ crosses both $F$ and $F^{\prime}$. Conversely, suppose that a hyperplane $H$ crosses $F$ and $F^{\prime}$, separating $x, y \in F^{\prime}$. Then $H$ cannot separate $x$ from $\mathfrak{g}_{F}(x)$ or $y$ from $\mathfrak{g}_{F}(y)$, so $H$ separates $\mathfrak{g}_{F}(x)$ and $\mathfrak{g}_{F}(y)$, and in particular crosses $\mathfrak{g}_{F}\left(F^{\prime}\right)$. Thus the set of hyperplanes crossing $\mathfrak{g}_{F}\left(F^{\prime}\right)$ is precisely the set of hyperplanes $H$ that cross both $F$ and $F^{\prime}$. Similarly, the set of hyperplanes crossing $\mathfrak{g}_{F^{\prime}}(F)$ is the set of hyperplanes $H$ that cross both $F$ and $F^{\prime}$. Hence $\mathfrak{g}_{F}\left(F^{\prime}\right), \mathfrak{g}_{F^{\prime}}(F)$ are parallel.

Suppose that $F \cap F^{\prime} \neq \varnothing$ and let $x \in F \cap F^{\prime}$. Then $\mathfrak{g}_{F}(x)=x$, by definition, so $\mathfrak{g}_{F}\left(F^{\prime}\right) \supseteq F \cap F^{\prime}$. On the other hand, let $y \in \mathfrak{g}_{F}\left(F^{\prime}\right)$, so $y=\mathfrak{g}_{F}\left(y^{\prime}\right)$ for some $y^{\prime} \in F^{\prime}$. Let $m$ be the median of $y, y^{\prime}, x$ for some $x \in F \cap F^{\prime}$. By convexity of $F$ and of $F^{\prime}$, we have $m \in F \cap F^{\prime}$ and $\mathrm{d}_{\mathcal{X}}\left(y, y^{\prime}\right) \geqslant \mathrm{d}_{\mathcal{X}}\left(y^{\prime}, m\right)$. Hence $y=m$, so $y \in F \cap F^{\prime}$. Thus $\mathfrak{g}_{F}\left(F^{\prime}\right) \subseteq F \cap F^{\prime}$, as required.

The following lemmas concern the projection of geodesics onto hyperplanes:

Lemma 2.7. Let $\alpha \subseteq \mathcal{X}$ be a combinatorial geodesic and let $K \subseteq \mathcal{X}$ be a convex subcomplex. Then $\mathfrak{g}_{K}(\alpha)$ is a geodesic in $K$. Moreover, suppose that there exists $R$ such that $\mathrm{d}_{\mathcal{X}}(a, K) \leqslant$ $R, \mathrm{~d}_{\mathcal{X}}\left(a^{\prime}, K\right) \leqslant R$ where $a, a^{\prime}$ are the initial and terminal 0 -cubes of $\alpha$. Then $\mathrm{d}_{\mathcal{X}}(t, K) \leqslant 2 R$ for all 0 -cubes $t$ of $\alpha$.

Proof. Let $\alpha^{\prime}=\mathfrak{g}_{K}(\alpha)$. Any hyperplane separating $t, t^{\prime} \in \alpha^{\prime}$ separates $s, s^{\prime} \in \alpha$ with $\mathfrak{g}_{K}(s)=$ $t, \mathfrak{g}_{K}\left(s^{\prime}\right)=t^{\prime}$, by the definition of gates. Hence the set of hyperplanes crossing $\alpha^{\prime}$ has the following properties: if $H, H^{\prime}$ cross $\alpha^{\prime}$ and are separated by $H^{\prime \prime}$, then $H^{\prime \prime}$ crosses $\alpha^{\prime}$; if $H, H^{\prime}, H^{\prime \prime}$ are pairwise-disjoint hyperplanes crossing $\alpha^{\prime}$, then one of them separates the other two. Hence, if $a_{0}, \ldots, a_{m}$ is an ordered sequence of 0 -cubes of $\alpha$, then $\mathfrak{g}_{K}\left(a_{0}\right), \ldots, \mathfrak{g}_{K}\left(a_{m}\right)$ has the property that $\mathfrak{g}_{K}\left(a_{i}\right), \mathfrak{g}_{K}\left(a_{i+1}\right)$ are either equal or adjacent for all $i$. If a hyperplane $H$ separates $\mathfrak{g}_{K}\left(a_{i}\right)$ from $\mathfrak{g}_{K}\left(a_{i+1}\right)$ and also separates $\mathfrak{g}_{K}\left(a_{j}\right)$ from $\mathfrak{g}_{K}\left(a_{j+1}\right)$, then $H$ must separate $a_{i}$ from $a_{i+1}$ and $a_{j}$ from $a_{j+1}$, a contradiction. This proves the first assertion.

To prove the second assertion, let $\alpha^{\prime}=\mathfrak{g}_{K}(\alpha)$. Let $\mathcal{H}$ be the set of hyperplanes separating $\alpha$ from $\mathfrak{g}_{K}(\alpha)$. For all $t \in \alpha$, any hyperplane $V$ separating $t$ from $K$ must not cross $K$. Hence $V$ separates $a$ or $a^{\prime}$ from $K$. The total number of such $V$ is at most $2 R-|\mathcal{H}|$.

2.2. Contact and crossing graphs and complexes. As before, $\mathcal{X}$ is an arbitrary CAT(0) cube complex and $\mathcal{C} \mathcal{X}$ its contact graph. Recall that hyperplanes $H, H^{\prime}$ represent adjacent vertices of $\mathcal{C X}$ if $\mathcal{N}(H) \cap \mathcal{N}\left(H^{\prime}\right) \neq \varnothing$, which occurs if either $H \cap H^{\prime} \neq \varnothing$ (in which case $H, H^{\prime}$ cross, denoted $H \perp H^{\prime}$ ), or if there are 1-cubes, dual to $H, H^{\prime}$ respectively, with a common 0 -cube that do not form the corner of a 2-cube, in which case $H, H^{\prime}$ osculate. If $H, H^{\prime}$ are adjacent in $\mathcal{C X}$, we say that they contact, denoted $H \pm H^{\prime}$. As we just did, we often abuse notation by saying a pair of hyperplanes are adjacent, when we really mean that the vertices represented by these hyperplanes are adjacent; similarly, we will talk about a sequence of 
hyperplanes forming a geodesic, etc. We sometimes refer to the crossing graph $\mathcal{C}_{\sharp} \mathcal{X}$ of $\mathcal{X}$, the spanning subgraph of $\mathcal{C} \mathcal{X}$ formed by removing open edges that record osculations.

The following statements are the beginning of the analogy between, on one hand, $\mathcal{X}$ and its contact graph and, on the other hand, $\mathcal{M C G}(S)$ and the curve complex of the surface $S$.

Theorem 2.8 (Hyperbolicity of contact graphs; [Hag14]). For any CAT(0) cube complex $\mathcal{X}$, there is a simplicial tree $T$ and a $(10,10)$-quasi-isometry $\mathcal{C} \mathcal{X} \rightarrow T$.

Under fairly mild geometric hypotheses, whether or not the quasi-tree $\mathcal{C X}$ is actually a quasi-point can be detected by examining the simplicial boundary; moreover, if $\mathcal{X}$ admits an essential, proper, cocompact group action, then $\mathcal{C X}$ is either a join of infinite subgraphs, or is unbounded [Hag13]. This is closely related to the action of rank-one elements on $\mathcal{C X}$ (recall that $g \in \operatorname{Aut}(\mathcal{X})$ is rank-one if it is hyperbolic and no axis bounds a half-flat in $\mathcal{X})$. As for the extension graph of a right-angled Artin group, there is a "Nielsen-Thurston classification" describing how elements of $\operatorname{Aut}(\mathcal{X})$ act on the contact graph.

Theorem 2.9 ("Nielsen-Thurston classification"; Hag13). Let $\mathcal{X}$ be a CAT(0) cube complex such that every clique in $\mathcal{C} \mathcal{X}$ is of uniformly bounded cardinality. Let $g \in \operatorname{Aut}(\mathcal{X})$. Then one of the following holds:

(1) ("Reducible") There exists $n>0$ and a hyperplane $H$ such that $g^{n} H=H$.

(2) ("Reducible") There exists a subgraph $\Lambda$ of $\mathcal{C X}$ such that $g \Lambda \subset \Lambda$ and the following holds: $\Lambda=\Lambda_{0} \sqcup \Lambda_{1}$, and each vertex of $\Lambda_{0}$ is adjacent to all but finitely many vertices of $\Lambda_{1}$. In this case, $g$ is not a rank-one element.

(3) ("Loxodromic") g has a quasigeodesic axis in $\mathcal{C X}$.

In the last case, $g$ is contracting and no positive power of $g$ stabilizes a hyperplane.

We will not use the subgraph $\Lambda$ in the rest of this paper; that description follows from Theorem D of Hag13. We will use the following: if $g \in \operatorname{Aut}(\mathcal{X})$ is not rank-one, then it either stabilizes a cube in $\mathcal{X}$ and thus has a bounded orbit in $\mathcal{C} \mathcal{X}$, or its combinatorial axis lies uniformly close to a half-flat $F$ in $\mathcal{X}$. As explained in [Hag13, Proposition 5.1], the set of hyperplanes crossing $F$ has uniformly bounded diameter in $\mathcal{C X}$, and hence the same is true of the $\langle g\rangle$ orbit in $\mathcal{C X}$.

2.3. Special cube complexes, right-angled Artin groups, and the extension graph. The right-angled Artin group $A_{\Gamma}$ presented by the graph $\Gamma$ is the group with presentation:

$\langle\operatorname{Vertices}(\Gamma)|\{[v, w]: v, w \in \operatorname{Vertices}(\Gamma),\{v, w\} \in \operatorname{Edges}(\Gamma\}\rangle$,

i.e., the generators are the vertices of $\Gamma$ and two generators commute if and only if the corresponding vertices span an edge of $\Gamma$. The Salvetti complex $S_{\Gamma}$ is the nonpositivelycurved cube complex with a single 0 -cube and an $n$-cube for each set of $n$ pairwise-commuting generators, for $n \geqslant 1$. Note that $S_{\Gamma}$ is compact, and $A_{\Gamma}$ finitely generated, if and only if $\Gamma$ is finite. See the survey [Cha07] for more details.

The universal cover $\widetilde{S}_{\Gamma}$ is a CAT $(0)$ cube complex with special features. For each induced subgraph $\Lambda$ of $\Gamma$, the inclusion $\Lambda \hookrightarrow \Gamma$ induces an injective local isometry $S_{\Lambda} \rightarrow S_{\Gamma}$, lifting to a $A_{\Lambda}$-equivariant convex embedding $\widetilde{S}_{\Lambda} \hookrightarrow \widetilde{S}_{\Gamma}$. In particular, when $\Lambda$ is the link of a vertex $v$, then $\widetilde{S}_{\Lambda}$ is a combinatorial hyperplane in $\widetilde{S}_{\Gamma}$ which is a copy of a hyperplane whose dual 1 -cubes are labeled by the generator $v$. Moreover, when $\Lambda$ is the star of $v$, the subcomplex $\widetilde{S}_{\Lambda} \cong \mathbf{R} \times \widetilde{S}_{\mathrm{Lk}(v)}$, where $\mathbf{R}$ is a convex subcomplex isometric to $\mathbb{R}$ and stabilized by $\langle v\rangle$.

The extension graph $\Gamma^{e}$ associated to $\Gamma$, introduced by Kim-Koberda in [KK13] is the graph with a vertex for each $A_{\Gamma}$ conjugate of each generator $v$ with $v \in \Gamma^{(0)}$ and an edge joining the distinct vertices corresponding to $v^{g}, w^{h}$ if and only if $\left[v^{g}, w^{h}\right]=1$. Like the contact graph of a cube complex, $\Gamma^{e}$ is always a quasi-tree [KK13], and in fact in many situations, $\Gamma^{e}$ is quasi-isometric to $\mathcal{C} \widetilde{S}_{\Gamma}$, as explained in [KK14]. 


\section{Part 1. Geometry of the contact graph}

We now discuss projection to the contact graph and identify "hierarchy paths" in a cube complex. These results will be reworked in Part 2 once we have introduced factor systems.

Disc diagram techniques, originating in unpublished notes of Casson and developed in [Che00, Sag95, Wis], have proven to be a useful tool in studying the geometry of $\mathcal{C X}$ (see [CH13, Hag14, Hag13), and we will continue to make use of these here.

\section{HiERARChY PATHS}

The next proposition establishes Theorem A. (2).

Proposition 3.1 ("Hierarchy paths"). Let $\mathcal{X}$ be a CAT(0) cube complex with contact graph $\mathcal{C X}$ and let $x, y \in \mathcal{X}$ be 0 -cubes. Then there exist hyperplanes $H_{0}, \ldots, H_{k}$ with $x \in \mathcal{N}\left(H_{0}\right), y \in$ $\mathcal{N}\left(H_{k}\right)$, and combinatorial geodesics $\gamma_{i} \rightarrow \mathcal{N}\left(H_{i}\right)$, such that $H_{0} \pm H_{1} \Psi \ldots \pm H_{k}$ is a geodesic of $\mathcal{C X}$ and $\gamma_{0} \gamma_{1} \cdots \gamma_{k}$ is a geodesic joining $x, y$.

Proof. Since the set of hyperplane carriers covers $\mathcal{X}$, there exist hyperplanes $H, H^{\prime}$ with $x \in \mathcal{N}(H), y \in \mathcal{N}\left(H^{\prime}\right)$. Let $k=d_{\mathcal{C X}}\left(H, H^{\prime}\right)$, and let $H=H_{0}, H_{1}, \ldots, H_{k}=H^{\prime}$ be a geodesic sequence of hyperplanes in $\mathcal{C X}$, so that $\mathcal{N}\left(H_{i}\right) \cap \mathcal{N}\left(H_{i+1}\right) \neq \varnothing$ for $0 \leqslant i \leqslant k-1$ and $\mathcal{N}\left(H_{i}\right) \cap \mathcal{N}\left(H_{j}\right)=\varnothing$ for $|i-j|>1$. Let $\gamma_{0}$ be a combinatorial geodesic of $\mathcal{N}\left(H_{0}\right)$ joining $x$ to a 0 -cube in $\mathcal{N}\left(H_{0}\right) \cap \mathcal{N}\left(H_{1}\right)$. For $1 \leqslant i \leqslant k-1$, let $\gamma_{i}$ be a geodesic of $\mathcal{N}\left(H_{i}\right)$ joining the terminal 0-cube of $\gamma_{i-1}$ to a 0 -cube of $\mathcal{N}\left(H_{i}\right) \cap \mathcal{N}\left(H_{i+1}\right)$. Finally, let $\gamma_{k}$ be a geodesic of $\mathcal{N}\left(H_{k}\right)$ joining the terminal 0 -cube of $\gamma_{k-1}$ to $y$. Since hyperplane carriers are convex subcomplexes of $\mathcal{X}$, each $\gamma_{i}$ is a geodesic of $\mathcal{X}$ and in particular contains at most one 1-cube dual to each hyperplane. Let $\gamma=\gamma_{0} \cdots \gamma_{k}$.

We now show that the above choices can be made in such a way that the path $\gamma$ is immersed in $\mathcal{X}$, i.e., $\gamma$ has no self-intersections. Since $\mathcal{N}\left(H_{i}\right) \cap \mathcal{N}\left(H_{j}\right)=\varnothing$ for $|i-j|>1$ we can restrict our attention to intersections between $\gamma_{i}$ and $\gamma_{i+1}$. Any such point of intersection must lie in $\mathcal{N}\left(H_{i}\right) \cap \mathcal{N}\left(H_{i+1}\right)$ and we can then replace $\gamma_{i}$ and $\gamma_{i+1}$ by $\gamma_{i}^{\prime}$ and $\gamma_{i+1}^{\prime}$, where $\gamma_{i}^{\prime} \subset \gamma_{i}$ and $\gamma_{i+1}^{\prime} \subset \gamma_{i+1}^{\prime}$ are the geodesic subpaths obtained by restricting to the subpath before, respectively, after, the intersection point. Applying this procedure for each $i$ to the first intersection point ensures that $\gamma$ is immersed.

Let $\tau$ be a combinatorial geodesic of $\mathcal{X}$ joining $y$ to $x$, so that $\gamma \tau$ is a closed path in $\mathcal{X}$ and let $D \rightarrow \mathcal{X}$ be a disc diagram with boundary path $\gamma \tau$. Suppose that $D$ has minimal area among all disc diagrams with this boundary path, and that the choice of geodesic of $\mathcal{C X}$, and the subsequent choice of paths $\gamma_{i}$, were made in such a way as to minimize the area of $D$. See Figure 1 .

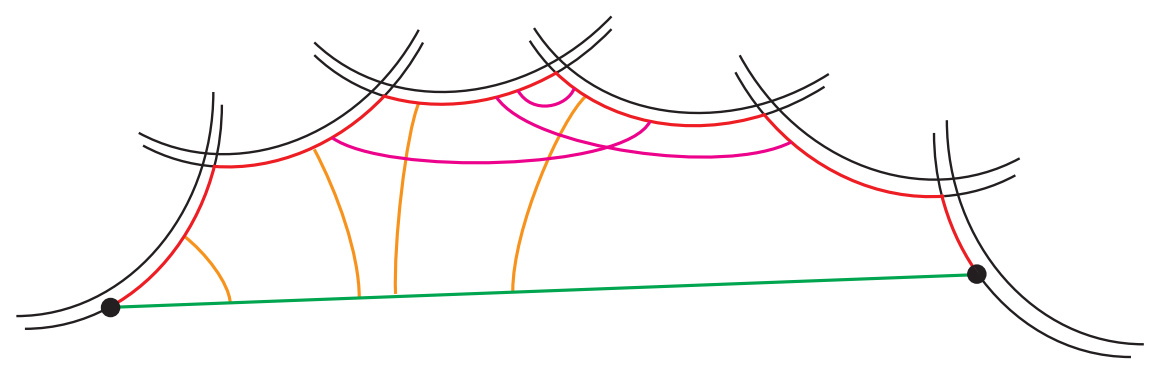

FiguRE 1. The diagram $D$, showing some possible (in orange) and impossible (in pink) dual curves.

Note that for all $i$, if $K, L$ are dual curves emanating from $\gamma_{i}$, then $K, L$ do not intersect. Otherwise, convexity of $\mathcal{N}\left(H_{i}\right)$ would enable a new choice of $\gamma_{i}$, lowering the area of $D$. 
Let $K$ be a dual curve in $D$ emanating from $\gamma$. It suffices to show that $K$ must end on $\tau$. Indeed, it will then follow that $|\gamma| \leqslant|\tau|$, whence $\gamma$ is a geodesic of the desired type. To this end, suppose that $K$ emanates from a 1-cube of $\gamma_{i}$. Then $K$ cannot end on $\gamma_{i}$, since $\gamma_{i}$ contains exactly one 1 -cube dual to the hyperplane to which $K$ maps. $K$ cannot end on $\gamma_{j}$ with $|i-j|>2$, for then $\mathcal{C} \mathcal{X}$ would contain a path $\left(H_{i}, V, H_{j}\right)$, where $V$ is the hyperplane to which $K$ maps, contradicting the fact that $d_{\mathcal{C X}}\left(H_{i}, H_{j}\right)=|i-j|$. If $K$ ends on $\gamma_{i \pm 2}$, then $\gamma_{i \pm 1}$ could be replaced by a path in $\mathcal{N}(V)$ that is the image of a path in the interior of $D$, contradicting our minimal-area choices. Indeed, recall that we chose the $\mathcal{C X}$-geodesic, and the associated geodesics $\gamma_{i}$, so that the resulting diagram $D$ (which can be constructed given any such choices) is of minimal area among all diagrams constructible by any choice of such $\mathcal{C X}$ and $\mathcal{X}$ geodesics.

Finally, suppose $K$ ends on $\gamma_{i+1}$. Then no dual curve emanating from the part of $\gamma_{i} \gamma_{i+1}$ subtended by the 1-cubes dual to $K$ can cross $K$. Hence the 1-cube of $\gamma_{i}$ dual to $K$ is equal to the 1-cube of $\gamma_{i+1}$ dual to $K$, contradicting the fact that $\gamma$ is immersed.

Definition 3.2. A geodesic $\gamma=\gamma_{1} \ldots \gamma_{n}$ such that $\gamma_{i} \rightarrow \mathcal{N}\left(H_{i}\right)$ for $1 \leqslant i \leqslant n$ and $H_{1} \pm \ldots \pm H_{n}$ is a geodesic of $\mathcal{C} \mathcal{X}$ is a hierarchy path. The geodesic $H_{1} \pm \ldots \pm H_{n}$ carries $\gamma$, and each $\gamma_{i}$ is a syllable of $\gamma$.

The following is immediate from the product structure of hyperplane-carriers:

Proposition 3.3. Let $\gamma=\gamma_{1} \cdots \gamma_{n}$ be a hierarchy path carried by $H_{1} \pm \ldots \pm H_{n}$. Then there exists a hierarchy path $\gamma^{\prime}=\gamma_{1}^{\prime} \cdots \gamma_{n}^{\prime}$, joining the endpoints of $\gamma$ and carried by $H_{1} \pm \ldots \pm H_{n}$, such that for each $i$, we have $\gamma_{i}^{\prime}=A_{i}$ e, where the combinatorial geodesic $A_{i}$ lies in $H_{i} \times\{ \pm 1\} \subset H_{i} \times[-1,1] \cong \mathcal{N}\left(H_{i}\right)$ and $e$ is either a 0 -cube or a 1-cube dual to $H_{i}$.

A hierarchy path satisfying the conclusion of Proposition 3.3 is reduced. A reduced hierarchy path $\gamma_{1} \cdots \gamma_{n}$ is a hereditary hierarchy path if it is trivial or if for each $i$, the subpath path $A_{i} \rightarrow H_{i} \times\{ \pm 1\}$ of $\gamma_{i}$ is a hereditary reduced hierarchy path in $H_{i} \times\{ \pm 1\}$. From Proposition 3.3 and the definition of a hereditary reduced hierarchy path, we obtain:

Corollary 3.4. Let $\mathcal{X}$ be a finite-dimensional $C A T(0)$ cube complex and let $x, y \in \mathcal{X}^{(0)}$. Then $x$ and $y$ are joined by a hereditary reduced hierarchy path.

\section{Projection to the contact Graph}

The notion of projecting geodesics in $\mathcal{X}$ to $\mathcal{C X}$ was discussed in [Hag13, Section 2], motivating the following definition:

Definition 4.1 (Projection to the contact graph). Let $K$ be a convex subcomplex of $\mathcal{X}$. For each $k \in K$, let $\left\{H_{i} \cap K\right\}_{i \in \mathcal{I}}$ be the maximal collection of hyperplanes of $K$ with $k \in$ $\cap_{i \in \mathcal{I}} \mathcal{N}\left(H_{i}\right)$. Define $\rho_{K}: K \rightarrow 2^{\mathcal{C} K}$ by setting $\rho_{K}(k)=\left\{H_{i}\right\}_{i \in \mathcal{I}}$. The projection of $\mathcal{X}$ on $\mathcal{C} K$ is the map $\pi_{K}=\rho_{K} \circ \mathfrak{g}_{K}: \mathcal{X} \rightarrow 2^{\mathcal{C} K}$. Note that $\pi_{K}$ assigns to each point of $\mathcal{X}$ a clique in the contact graph of $K$ and hence a clique in $\mathcal{C X}$.

If $H$ is a hyperplane, let $H^{ \pm}$be $H \times\{ \pm 1\} \subset \mathcal{N}(H) \cong H \times[-1,1]$. There is a bijection between hyperplanes which intersect $H^{+}$and ones which intersect $H^{-}$; moreover, associated hyperplanes contact [respectively, cross] in $H^{+}$if and only if they contact [respectively, cross] in $H^{-}$, whence $\mathcal{C} H^{+}$and $\mathcal{C} H^{-}$are both the same subset of $\mathcal{C X}$. Abusing notation slightly, we let $\pi_{H}$ denote the projection from $\mathcal{X}$ to $\mathcal{C} H^{+}=\mathcal{C} H^{-} \subset \mathcal{C X}$. This map is defined as in Definition 4.1 since $H^{ \pm}$is a convex subcomplex, and it is not hard to see that it is independent of whether we took gates in $\mathrm{H}^{+}$or $\mathrm{H}^{-}$(another option is to pass to the first cubical subdivision and just project to $H$ and then to its contact graph, since subdividing makes $H$ into a subcomplex). 
Let $x, y \in \mathcal{X}$ be 0 -cubes, and let $H$ be a hyperplane that does not separate $x$ from $y$. Let $H^{+}$be the copy of $H$ bounding $\mathcal{N}(H)$ that lies in the component of $\mathcal{X}-H$ containing $\{x, y\}$. It is easily checked that any hyperplane that separates $\mathfrak{g}_{H^{+}}(x)$ from $\mathfrak{g}_{H^{+}}(y)$ must separate $x$ from $y$. In particular, if $H$ does not separate $x$ from $y$ and it does not cross any hyperplane separating $x$ from $y$, then $\pi_{H}(x)=\pi_{H}(y)$. In other words, if $\gamma \subset \mathcal{X}$ is a geodesic with $\pi_{\mathcal{X}}(\gamma) \cap B_{1}^{\mathcal{C}_{\sharp} \mathcal{X}}(H)=\varnothing$, then $\pi_{H}(x)=\pi_{H}(y)$, where $x, y$ are the endpoints of $\gamma$. Let $x^{\prime} \in \gamma$ be a 0-cube and suppose that the hyperplane $U$ crosses $H$ and separates the pair $x, x^{\prime}$, hence separating $\mathfrak{g}_{H^{+}}(x)$ from $\mathfrak{g}_{H^{+}}\left(x^{\prime}\right)$. Then $U$ separates $x, y$, and hence belongs to $\pi_{\mathcal{X}}(\gamma) \cap B_{1}^{\mathcal{C}_{\sharp} \mathcal{X}}(H)$, contradicting our assumption. Hence $\pi_{H}(\gamma)=\pi_{H}(x)=\pi_{H}(y)$. Thus:

Proposition 4.2 (Bounded geodesic image). For any $H \in \mathcal{C X}$ and any geodesic $\gamma \subseteq \mathcal{X}$ whose image in $\mathcal{C X}$ is disjoint from $B_{1}^{\mathcal{C}_{\sharp} \mathcal{X}}(H)$, the projection $\pi_{H}(\gamma)$ is a clique.

\section{WEAK PROPER DiscontinUity of the ACTION ON THE CONTACT GRAPH}

We now consider the elements which act weakly properly discontinuously (WPD), in the sense of [BF02]. In particular, we study the WPD elements of a group $G$ acting on $\mathcal{X}$, and prove Theorem A.(1). By definition, as an isometry of $\mathcal{C} \mathcal{X}$, some $h \in G$ is WPD if for all $\epsilon \geqslant 1$ and hyperplanes $H$, there exists $N>0$ such that

$$
\left|\left\{g \in G: \mathrm{d}_{\mathcal{C X}}(H, g H)<\epsilon, \mathrm{d}_{\mathcal{C X}}\left(h^{N} H, g h^{N} H\right)<\epsilon\right\}\right|<\infty .
$$

In the presence of a factor system and a cocompact group action, we achieve a stronger conclusion in Section 14, namely that the action on the contact graph is acylindrical.

Proposition 5.1. Let $\mathcal{X}$ be a $C A T(0)$ cube complex on which the group $G$ acts metrically properly by isometries. Suppose that $h \in G$ is loxodromic on $\mathcal{C X}$. Then $h$ is $W P D$.

Proof. Fix $\epsilon \geqslant 1$ and let $H$ be a hyperplane. By hypothesis, $\langle h\rangle H$ is a quasigeodesic in $\mathcal{C X}$. It follows, c.f., Hag13, Theorem 2.3], that there exists $M=M(h, H, \mathcal{X})$ such that for all $N \geqslant 0$, and any hierarchy path $\gamma=\gamma_{1} \cdots \gamma_{p}$ joining some $x \in H$ to $h^{N} x \in h^{N} H$ and carried on a geodesic $H=H_{0} \pm H_{1} \Psi \ldots \pm H_{p}=h^{N} H$ of $\mathcal{C X}$, we have $\left|\gamma_{i}\right| \leqslant M$ for $0 \leqslant i \leqslant p$. (Recall that $\gamma$ is a geodesic, being a hierarchy path; hence $\gamma$ contains at most one 1 -cube dual to each hyperplane.)

Suppose that $g \in G$ has the property that $\mathrm{d}_{\mathcal{C X}}(H, g H)<\epsilon$ and $\mathrm{d}_{\mathcal{C X}}\left(h^{N} H, g h^{N} H\right)<\epsilon$. Suppose, moreover, that $N$ has been chosen so that $p>2 \epsilon+12$ and choose $i$ so that $\left|\frac{p}{2}-i\right| \leqslant 2$. Choose a point $y \in \gamma_{i}$ and let $\mathcal{H}(y)$ be the set of hyperplanes separating $y$ from $g y \in g \gamma_{i}$.

We claim that each $W \in \mathcal{H}(y)$ intersects $\gamma$ and $g \gamma$. Indeed, suppose that $W$ intersects $\gamma$ but not $g \gamma$. Suppose that $W$ intersects $\gamma_{j}$ with $j \geqslant i$. Then $W$ separates $x, h^{N} x$ (since $\gamma$ is a geodesic) but not $g x, g h^{N} x$, so $W$ separates $x, g x$ or $h^{N} x, g h^{N} x$. Since $W$ also separates $y, g y$, the former must hold. Hence $p=\mathrm{d}_{\mathcal{C X}}\left(H, h^{N} H\right) \leqslant \epsilon+|p-i| \leqslant p / 2+\epsilon$, contradicting our choice of $N$. The case where $W$ intersects $\gamma_{j}, j<i$ is similar.

Hence, if $W$ crosses $\gamma_{s}$ with $s \geqslant i$, then $W$ crosses $g \gamma_{t}$, with $t \leqslant i$. (We have the reverse conclusion if $s \leqslant i$.) The fact that $H_{0} \pm H_{1} \Psi \ldots \pm H_{p}$ and its $g$-translate are $\mathcal{C} \mathcal{X}$-geodesics implies that $W$ intersects $\gamma_{s}$ with $|i-s| \leqslant \epsilon+6$. Hence $\mathrm{d}_{\mathcal{X}}(y, g y) \leqslant M(\epsilon+6)$, whence the number of such $g$ is finite since the action of $G$ on $\mathcal{X}$ is proper.

We say that a group is nonelementary if it does not contain a cyclic subgroup of finite index, and that an action of the group $G$ is WPD if it admits a WPD element and $G$ is nonelementary.

Corollary 5.2 (Characterization of WPD elements). Let $\mathcal{X}$ be a uniformly locally finite $C A T(0)$ cube complex. Then for any nonelementary group $G$ acting properly on $\mathcal{X}$, one of the following holds: 
(1) The induced action of $G$ on $\mathcal{C X}$ is $W P D$, and the WPD elements are precisely those $h \in G$ that are rank-one and do not have positive powers that stabilize hyperplanes.

(2) Every $h \in G$ is either not rank-one, or has a positive power stabilizing a hyperplane.

Proof. Apply Proposition 5.1 and Theorem 2.9.

The following is an application to determining acylindrical hyperbolicity. Following [CS11], we say that the action of the group $G$ on the $\mathrm{CAT}(0)$ cube complex $\mathcal{X}$ is essential if every halfspace contains points in a fixed $G$-orbit arbitrarily far away from the associated hyperplane.

Corollary 5.3. Let $G$ be a nonelementary group acting properly and essentially on a uniformly locally finite $C A T(0)$ cube complex, $\mathcal{X}$. Suppose that $\mathcal{X}$ is not a product of unbounded subcomplexes and at least one of the following two holds:

(1) $G$ acts cocompactly on $\mathcal{X}$.

(2) $G$ acts with no fixed point in the simplicial boundary $\partial_{\triangle} \mathcal{X}$ in the sense of [Hag13].

Then $G$ is acylindrically hyperbolic.

Proof. By [Osi14, Theorem 1.2] it suffices to find a hyperbolic space on which $G$ acts with a loxodromic WPD element. By [Hag13, Theorem 5.4] there exists a rank-one element $g \in G$ no positive power of which stabilizes a hyperplane. The conclusion follows from Corollary 5.2 .

A major motivation for studying WPD actions arises from a result of Bestvina-Fujiwara relating WPD actions to bounded cohomology. Recall that the space $\widetilde{Q H}(G)$, which is the quotient of the space of quasimorphisms of $G$ by the subspace generated by bounded functions and homomorphisms $G \rightarrow \mathbb{R}$, coincides with the kernel of the $\operatorname{map}_{b}^{2}(G, \mathbb{R}) \rightarrow$ $\mathrm{H}^{2}(G, \mathbb{R})$. Theorem 7 of $\mathrm{BF} 02$ asserts that if $G$ admits a WPD action, then $\widetilde{Q H}(G)$ is infinite-dimensional. This yields an alternative proof of the dichotomy obtained by CapraceSageev in [CS11, Theorem H], as a consequence of rank-rigidity: a group $G$ admitting a sufficiently nice action on a $\mathrm{CAT}(0)$ cube complex $\mathcal{X}$ that is not a product has infinitedimensional $\widetilde{Q H}(G)$; instead of using rank-rigidity and results of [BM02, CM09, Mon01, one can deduce their result from rank-rigidity, Corollary 5.2 , and [BF02].

\section{Contractibility}

We now prove Theorem A. (3). The contact complex $\mathcal{C} \cdot \mathcal{X}$ of the CAT(0) cube complex $\mathcal{X}$ is the flag complex with 1-skeleton $\mathcal{C X}$.

Theorem 6.1. Let $\mathcal{X}$ be a $C A T(0)$ cube complex with countable 0-skeleton and at least one 1-cube. Then $\mathcal{C} . \mathcal{X}$ is contractible.

Proof. For each $n \geqslant 0$, choose a convex, compact subcomplex $\mathcal{B}_{n}$ in such a way that $\mathcal{B}_{m} \subseteq \mathcal{B}_{n}$ for $m \leqslant n$ and $\cup_{n \geqslant 0} \mathcal{B}_{n}=\mathcal{X}$. This choice is possible since $\mathcal{X}^{(0)}$ may be written as an increasing union of finite sets; $\mathcal{B}_{n}$ can then be taken to be the cubical convex hull of the $n^{\text {th }}$ one.

Since each $\mathcal{B}_{n}$ is convex in $\mathcal{X}$, it is itself a $\operatorname{CAT}(0)$ cube complex whose hyperplanes have the form $H \cap \mathcal{B}_{n}$, where $H$ is a hyperplane of $\mathcal{X}$. Moreover, the map $H \cap \mathcal{B}_{n} \rightarrow H$ induces an embedding $\mathcal{C}_{\bullet} \mathcal{B}_{n} \rightarrow \mathcal{C} \bullet \mathcal{X}$ whose image is a full subcomplex (i.e., any $k+10$-simplices of $\mathcal{C}_{\bullet} \mathcal{B}_{n}$ span a $k$-simplex of $\mathcal{C} \cdot \mathcal{B}_{n}$ if and only if their images in $\mathcal{C}_{\bullet} \mathcal{X}$ span a $k$-simplex of $\mathcal{C} \cdot \mathcal{X}$ ). Thus the set $\left\{\mathcal{C}_{\bullet} \mathcal{B}_{n}\right\}_{n \geqslant 0}$ provides a filtration of $\mathcal{C} \cdot X$ by full subcomplexes, each of which is the contact complex of a compact CAT(0) cube complex. Indeed, every hyperplane intersects all but finitely many of the $\mathcal{B}_{n}$, and hence appears as a 0 -simplex in all but finitely many of the subcomplexes $\mathcal{C}_{\bullet} \mathcal{B}_{n}$. 
For any $m \geqslant 0$ and any continuous map $f: \mathbb{S}^{m} \rightarrow \mathcal{C} \cdot \mathcal{X}$, compactness of im $f$ implies that there exists $n \geqslant 0$ such that $\operatorname{im} f \subseteq \mathcal{C} . \mathcal{B}_{n}$. By Lemma 6.2, $\mathcal{C}_{\bullet} \mathcal{B}_{n+1}$ is contractible, since it is the contact complex of a compact $\mathrm{CAT}(0)$ cube complex. Hence the inclusion $\mathcal{C}_{\bullet} \mathcal{B}_{n} \hookrightarrow \mathcal{C} \bullet \mathcal{B}_{n+1}$

is null-homotopic, whence $\mathbb{S}^{m} \stackrel{f}{\longrightarrow} \mathcal{C}_{\bullet} \mathcal{B}_{n} \hookrightarrow \mathcal{C} \bullet \mathcal{B}_{n+1} \hookrightarrow \mathcal{C} \bullet \mathcal{X}$ is null-homotopic. It then follows from Whitehead's theorem [Whi49a, Whi49b] that $\mathcal{X}$ is contractible.

Lemma 6.2. Let $\mathcal{X}$ be a compact $C A T(0)$ cube complex with at least one 1-cube. Then $\mathcal{C} \cdot \mathcal{X}$ is contractible.

Proof. We will argue by induction on $n=\left|\mathcal{C}_{\bullet} \mathcal{X}^{(0)}\right|$, i.e., the number of hyperplanes in $\mathcal{X}$. When $n=1$, the cube complex $\mathcal{X}$ is necessarily a single 1 -cube, so $\mathcal{C} \cdot \mathcal{X}$ is a 0 -simplex. Let $n \geqslant 1$. Since $\mathcal{X}$ has finitely many hyperplanes, there exists a hyperplane $H \subset \mathcal{X}$ such that one of the components of $\mathcal{X}-H$ has closure $H \times[0,1]$, where $\mathcal{N}(H)$ is identified with $H \times[-1,1]$ and $H$ with $H \times\{0\}$. This generalizes the case in which $\mathcal{X}$ is a tree and $H$ is the midpoint of an edge containing a degree-1 vertex; accordingly, such a hyperplane $H$ will be called a leaf hyperplane. When $H$ is a leaf hyperplane, we always denote by $H^{+}$the halfspace $H \times[0,1]$ and by $H^{-}$the other closed halfspace.

By Lemma 6.3. $\mathcal{A}=C l(\mathcal{X}-\mathcal{N}(H))$ is a convex proper subcomplex of $\mathcal{X}$, so that $\mathcal{C} . \mathcal{A}$ embeds in $\mathcal{C} . \mathcal{X}$ as a full subcomplex. Moreover, by Lemma 6.4 there is a convex proper subcomplex $H^{\prime} \subset \mathcal{X}$ such that $\mathcal{C} \cdot \mathcal{X} \cong \mathcal{C} \cdot \mathcal{A} \cup_{L}(L \star\{H\})$, where $L$ is a subcomplex of $\mathcal{C} . C l(\mathcal{X}-\mathcal{N}(H))$ isomorphic to $\mathcal{C} . H^{\prime}$. Each of $\mathcal{A}$ and $H^{\prime}$ is a $\operatorname{CAT}(0)$ cube complex whose set of hyperplanes corresponds bijectively to a subset of $\mathcal{X}^{(0)}-\{H\}$, so by induction, $\mathcal{C} \cdot \mathcal{A}$ and $L$ are contractible. Since $L$ is contractible, there is a homotopy equivalence $f: L \star\{H\} \rightarrow L$ that is the identity on $L$, whence the pasting lemma yields a homotopy equivalence $\mathcal{C} \cdot \mathcal{X} \rightarrow \mathcal{C} \cdot \mathcal{A}$. Since $\mathcal{A}$ is contractible, the same is therefore true of $\mathcal{C} . \mathcal{X}$.

Lemma 6.3. Let $H$ be a leaf hyperplane of the $C A T(0)$ cube complex $\mathcal{X}$. Then $C l(\mathcal{X}-\mathcal{N}(H))$ is a convex subcomplex of $\mathcal{X}$.

Proof. Since $H^{+} \subset \mathcal{N}(H)$, the subcomplex $C l(\mathcal{X}-\mathcal{N}(H))$ is exactly the convex hull of the halfspace $H^{-} \cap \mathcal{X}^{(0)}$ of the 0 -skeleton induced by $H$.

Lemma 6.4. Let $H$ be a leaf hyperplane of the compact $C A T(0)$ cube complex $\mathcal{X}$. Then there is convex subcomplex $H^{\prime} \subsetneq \mathcal{X}$ such that there is an isomorphism

$$
\mathcal{C}_{\bullet} \mathcal{X} \cong \mathcal{C} \cdot C l(\mathcal{X}-\mathcal{N}(H)) \cup_{L}(L \star\{H\}),
$$

where $L$ is a subcomplex of $\mathcal{C} \cdot C l(\mathcal{X}-\mathcal{N}(H))$ isomorphic to $\mathcal{C} \cdot H^{\prime}$.

Proof. Let $\mathcal{A}=C l(\mathcal{X}-\mathcal{N}(H))$. Let $\left\{V_{1}, \ldots, V_{s}\right\}$ be the hyperplanes of $\mathcal{X}$ that contact $H$, and let $L$ be the full subcomplex of $\mathcal{C} \cdot \mathcal{X}$ generated by $\left\{V_{1}, \ldots, V_{s}\right\}$. Lemma 7.11 of [Hag14] implies that there is an isometrically embedded subcomplex $H^{\prime} \subset \mathcal{X}$ such that a hyperplane intersects $H^{\prime}$ if and only if that hyperplane belongs to $\left\{V_{1}, \ldots, V_{k}\right\}$. By replacing $H^{\prime}$ if necessary by its convex hull, we may assume that $H^{\prime}$ is convex, and hence $\mathcal{C}_{\bullet} H^{\prime} \cong L$. The decomposition is obvious from the definitions.

\section{Automorphisms of the COntact Graph}

We now provide a short example which shows that the analogue of Ivanov's Theorem, that the mapping class group is the automorphism group of the curve graph [Iva97], fails to hold for the contact graph. Indeed, the example below shows that this failure holds even if one considers an edge-colored version of the contact graph differentiating edges associated to crossing hyperplanes from those associated to osculating ones. We note that in the case of RAAGs, the fact that the automorphism group of the contact graph may be much larger than 
the RAAG itself is familiar from the case of the extension graph, where Kim-Koberda prove that the automorphism of the extension graph is often uncountable [KK14, Theorem 66].

Example 7.1. In the cube complex pictured in Figure 7.1 one sees that the automorphism group of the cube complex is virtually cyclic, and in particular, pairs of valence zero vertices, such as those labelled $a, b$ can not be permuted willy-nilly. Whereas in the contact graph, the pair of simplices (labelled $a^{\prime}, b^{\prime}$ ), one for each hyperplane separating off such a vertex, can be swapped arbitrarily; thus the contact graph contains a $(\mathbb{Z} / 2 \mathbb{Z})^{\infty}$ subgroup.
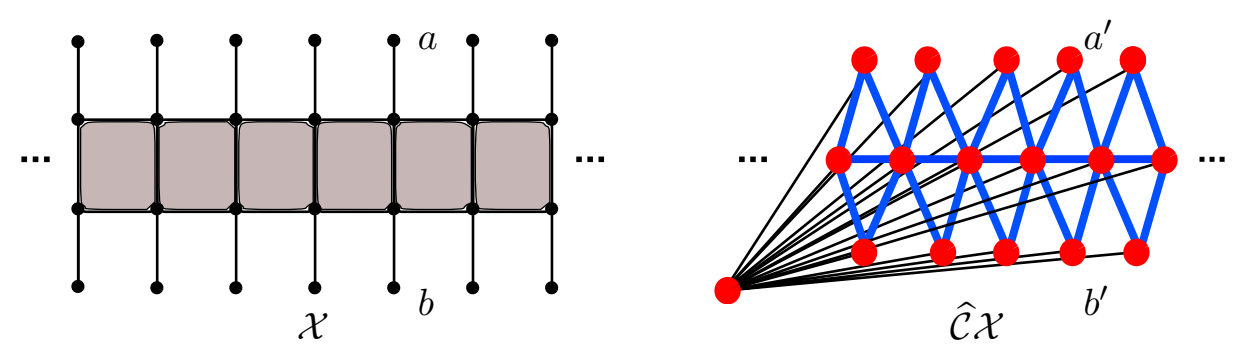

Figure 2. Cube complex drawn at left; its associated contact graph at right.

\section{Part 2. Factor systems, projections, and the distance formula}

Throughout, $\mathcal{X}$ is a $\operatorname{CAT}(0)$ cube complex. The setting in which we will work is established in Definition 8.1 and forces $\mathcal{X}$ to be uniformly locally finite.

\section{FACTORED CONTACT GRAPHS}

\subsection{Factor systems.}

Definition 8.1 (Factor system). A set of subcomplexes, $\mathfrak{F}$, which satisfies the following is called a factor system in $\mathcal{X}$ :

(1) $\mathcal{X} \in \mathfrak{F}$.

(2) Each $F \in \mathfrak{F}$ is a nonempty convex subcomplex of $\mathcal{X}$.

(3) There exists $\Delta \geqslant 1$ such that for all $x \in \mathcal{X}^{(0)}$, at most $\Delta$ elements of $\mathfrak{F}$ contain $x$.

(4) Every nontrivial convex subcomplex parallel to a combinatorial hyperplane of $\mathcal{X}$ is in $\mathfrak{F}$.

(5) There exists $\xi \geqslant 0$ such that for all $F, F^{\prime} \in \mathfrak{F}$, either $\mathfrak{g}_{F}\left(F^{\prime}\right) \in \mathfrak{F}$ or $\operatorname{diam}\left(\mathfrak{g}_{F}\left(F^{\prime}\right)\right)<\xi$.

Convexity of each $F \in \mathfrak{F}$ implies that each $F$ is a CAT(0) cube complex whose hyperplanes have the form $H \cap F$, where $H$ is a hyperplane of $\mathcal{X}$, and the map $H \cap F \mapsto H$ induces an injective graph homomorphism $\mathcal{C} F \hookrightarrow \mathcal{C X}$ whose image is an induced subgraph, which, by an abuse of notation, we also denote $\mathcal{C} F$.

If $\mathfrak{F}$ is a factor system for $\mathcal{X}$, then for each $F \in \mathfrak{F}$, the set $\mathfrak{F}_{F}=\{H \in \mathfrak{F}: H \subseteq F\}$ is a factor system in $F$ such that each point in $F$ lies in at most $\Delta-1$ elements of $\mathfrak{F}_{F}$. (The distinction between $\mathfrak{F}_{F}$ and $\{H \cap F: H \in \mathfrak{F}\}$ is small: the latter consists of the former, together with some subcomplexes of diameter at most $\xi$. It is mainly for convenience that we choose to work, everywhere, with $\mathfrak{F}_{F}$ rather than $\{H \cap F: H \in \mathfrak{F}\}$.)

\subsection{Examples of factor systems.}


8.2.1. Special groups. Universal covers of special cube complexes, defined in [HW08], contain factor systems provided the co-special action has finitely many orbits of hyperplanes, as we shall show below. This provides a very large class of groups for which the distance formula from Section 9 holds. Let us start by studying universal covers of Salvetti complexes.

The following definition is tailored to the proof of Proposition 8.3 .

Definition 8.2. Let $\Gamma$ be a simplicial graph. A collection $\mathcal{R}$ of subgraphs of $\Gamma$ is rich if

(1) $\Gamma \in \mathcal{R}$,

(2) all links of vertices of $\Gamma$ are in $\mathcal{R}$, and

(3) if $A, B \in \mathcal{R}$ then $A \cap B \in \mathcal{R}$.

The collection of all subgraphs of $\Gamma$ is a rich family. Also, any graph admits a minimal rich family, consisting of $\Gamma$ together with all nonempty intersections of links of vertices.

Proposition 8.3. Let $\Gamma$ be a finite simplicial graph, $A(\Gamma)$ a right-angled Artin group, and $\widetilde{S}_{\Gamma}$ the universal cover of its Salvetti complex. Let $\mathcal{R}$ be a rich family of subgraphs of $\Gamma$.

Let $\mathfrak{F}$ be the $\left(A(\Gamma)\right.$-invariant) collection of convex subcomplexes of $\widetilde{S}_{\Gamma}$ containing all lifts of the sub-Salvetti complexes $S_{\Lambda}$ of $S_{\Gamma}$, for all $\Lambda \in \mathcal{R}$. Then $\mathfrak{F}$ is a factor system.

In other words, $\mathfrak{F}$ contains a subcomplex stabilized by each conjugate of $A(\Lambda)$, for each subgraph $\Lambda$ of $\Gamma$.

Proof of Proposition 8.3. In the definition of a factor system, item (1) holds because $\Gamma \in \mathcal{R}$. Items (2) and (3) are clear. Item (4) holds because combinatorial hyperplanes in $\widetilde{S}_{\Gamma}$ are exactly lifts of sub-Salvetti complexes of $S_{\Gamma}$ corresponding to links of vertices. More precisely, if a hyperplane $H$ is dual to a 1-cube corresponding to the generator $v \in \Gamma^{(0)}$, then the combinatorial hyperplanes on the two sides of $H$ are lifts of $S_{\mathrm{Lk}(v)} \subseteq S_{\Gamma}$.

The content of the proposition is hence that Item (5) holds (for $\xi=0$ ). To simplify the notation, we will identify the 0 -skeleton of $\widetilde{S}_{\Gamma}$ with $A(\Gamma)$. Under this identification, the 0skeleton of each $F \in \mathfrak{F}$ corresponds to a coset of $A(\Lambda)<A(\Gamma)$ for some subgraph $\Lambda \in \mathcal{R}$, and each such coset is the 0 -skeleton of some $F \in \mathfrak{F}$.

Let $F, F^{\prime} \in \mathfrak{F}$, whose 0 -skeleta are (possibly after applying an element of $\left.A(\Gamma)\right) A\left(\Lambda_{0}\right)$ and $g A\left(\Lambda_{1}\right)$, for some subgraphs $\Lambda_{0}, \Lambda_{1}$ of $\Gamma$ and $g \in A(\Gamma)$. We can assume, using the action of some $g \in A\left(\Gamma_{0}\right)$, that $1 \in \mathfrak{g}_{F}\left(F^{\prime}\right)$. Recall from Lemma 2.4 and Lemma 2.6 that $\mathfrak{g}_{F}\left(F^{\prime}\right)$ is in a natural way one of the factors in a product region $R$ of $\widetilde{S}_{\Gamma}$, the other factor being (naturally identified with) a possibly trivial geodesic $\gamma$ from 1 to $g$, up to changing the choice of $g$ within the same coset of $A\left(\Lambda_{1}\right)$. Also, $\mathfrak{g}_{F}\left(F^{\prime}\right) \times\{1\}$ is contained in $F$ and $\mathfrak{g}_{F}\left(F^{\prime}\right) \times\{g\}$ is contained in $F^{\prime}$. Let $\Lambda_{2}$ be the link in $\Lambda$ of the set of vertices of $\Lambda$ that label some 1-cube along $\gamma$. The following claim concludes the proof.

Claim: The 0-skeleton of $\mathfrak{g}_{F}\left(F^{\prime}\right)$ is $A(\Lambda)$, where $\Lambda=\bigcap_{i=0,1,2} \Lambda_{i} \in \mathcal{R}$.

Let us first show $\mathfrak{g}_{F}\left(F^{\prime}\right)^{(0)} \subseteq A(\Lambda)$. Consider a geodesic $\delta$ joining 1 to $h \in \mathfrak{g}_{F}\left(F^{\prime}\right)^{(0)}$. Any 1-cube $e$ of $\delta$ is contained in $F$ as well as parallel to a 1-cube of $F^{\prime}$, which implies that the label $v$ of $e$ belongs to $\Lambda_{0} \cap \Lambda_{1}$. Let us now show that $e$ also belongs to $\Lambda_{2}$. Once we have done that, it is clear that $h$ can be written as a product of generators each belonging to $A(\Lambda)$.

Fix any 1-cube $e^{\prime}$ of $\gamma$. The product region $R$ contains a square with two 1-cubes parallel to $e$ and two 1-cubes parallel to $e^{\prime}$. This means that the labels of $e, e^{\prime}$ commute and are distinct. As this holds for any $e^{\prime}$, the label of $e$ belongs to $\Lambda_{2}$, as required.

We are left to show $A(\Lambda) \subseteq \mathfrak{g}_{F}\left(F^{\prime}\right)^{(0)}$. If $h \in A(\Lambda)$, there exists a geodesic $\delta$ from 1 to $h$ whose 1 -cubes are labeled by elements of $\gamma$. It is then easy to see that $\widetilde{S}_{\Gamma}$ (and in fact $R$ ) contains a product region naturally identified with $\delta \times \gamma$ with the property that $\delta \times\{1\}$ is contained in $F$ and $\delta \times\{g\}$ is contained in $F^{\prime}$. In particular, $\delta$, and thus $h$, is contained in $\mathfrak{g}_{F}\left(F^{\prime}\right)$, as required. 
Definition 8.4 (Induced factor system). Let $\mathcal{X}$ be a CAT(0) cube complex with a factor system $\mathfrak{F}$ and let $\mathcal{Y} \subseteq \mathcal{X}$ be a convex subcomplex. The induced factor system $\mathfrak{F} y$ be the set of nonempty subcomplexes of $\mathcal{Y}$ of the form $F \cap \mathcal{Y}, F \in \mathfrak{F}$.

Lemma 8.5. Let $\mathcal{X}$ be a CAT(0) cube complex with a factor system $\mathfrak{F}$ and let $\mathcal{Y} \subseteq \mathcal{X}$ be a convex subcomplex. Then $\mathfrak{F} y$ is a factor system in $\mathcal{Y}$.

Proof. Item (1) of Definition 8.1 holds since $\mathcal{X} \cap \mathcal{Y}=\mathcal{Y}$. Item 2 follows since intersections of convex subcomplexes are convex. Item (3) follows since $\mathfrak{F}$ is a uniformly locally finite collection. To verify Item (4), let $H^{+}$be a combinatorial hyperplane of $\mathcal{Y}$ and let $H$ be the (genuine) hyperplane whose carrier contains $H^{+}$as one of its bounding copies. By convexity of $\mathcal{Y}$, the hyperplane $H$ has the form $W \cap \mathcal{Y}$ for some hyperplane $W$ of $\mathcal{X}$, and hence $H^{+}=W^{+} \cap \mathcal{Y}$, where $W^{+}$is one of the combinatorial hyperplanes bounding the carrier of $W$. But $W^{+} \in \mathfrak{F}$, by item (4), so $W^{+} \cap \mathcal{Y}=H^{+} \in \mathfrak{F} \mathcal{Y}$.

It suffices to verify item (5), namely that $\mathfrak{F} y$ is closed under (large) projection. To that end, let $F, F^{\prime} \in \mathfrak{F}$ and suppose that $\operatorname{diam}\left(\mathfrak{g}_{F \cap \mathcal{Y}}\left(F^{\prime} \cap \mathcal{Y}\right)\right) \geqslant \xi$, where $\xi$ is the constant associated to $\mathfrak{F}$ by Definition 8.1. Then, by item (5), applied to $\mathfrak{F}$, we have $\mathfrak{g}_{F}\left(F^{\prime}\right) \in \mathfrak{F}$. It thus suffices to show that $\mathfrak{g}_{F}\left(F^{\prime}\right) \cap \mathcal{Y}=\mathfrak{g}_{F \cap \mathcal{Y}}\left(F^{\prime} \cap \mathcal{Y}\right)$. For convenience, let $A=F \cap \mathcal{Y}, A^{\prime}=F^{\prime} \cap \mathcal{Y}$. Since $A \subseteq \mathcal{Y}$, we have $\mathfrak{g}_{A}\left(A^{\prime}\right) \subseteq \mathcal{Y}$. Since $A \subseteq F$ and $A^{\prime} \subseteq F^{\prime}$, we have $\mathfrak{g}_{A}\left(A^{\prime}\right) \subseteq \mathfrak{g}_{F}\left(F^{\prime}\right)$. Hence $\mathfrak{g}_{A}\left(A^{\prime}\right) \subseteq \mathfrak{g}_{F}\left(F^{\prime}\right) \cap \mathcal{Y}$.

Conversely, suppose that $x \in \mathfrak{g}_{F}\left(F^{\prime}\right) \cap \mathcal{Y}$. Then $x \in A$, since $x \in F \cap \mathcal{Y}$. Since $x \in \mathfrak{g}_{F}\left(F^{\prime}\right)$, there exists $x^{\prime} \in F^{\prime}$ such that a hyperplane $H$ separates $x^{\prime}$ from $F$ if and only if $H$ separates $x^{\prime}$ from $x$. Let $z=\mathfrak{g} y\left(x^{\prime}\right)$. Note that convexity of $\mathcal{Y}$ is used here to make $z$ well-defined. Let $V$ be a hyperplane separating $z$ from $x$. Then either $V$ separates $x^{\prime}$ from $x$, and hence from $F$, whence $V$ separates $z$ from $A$, or $V$ separates $x, x^{\prime}$ from $z$. Suppose the latter. Since $V$ separates $x^{\prime}$ from $z$, it must separate $x^{\prime}$ from $Y$ since $z=\mathfrak{g}_{\mathcal{Y}}\left(x^{\prime}\right)$. But then $V$ cannot cross $\mathcal{Y}$, and hence cannot separate $z \in \mathcal{Y}$ from $x \in \mathcal{Y}$, a contradiction. Thus $V$ separates $z$ from $x$ if and only if $V$ separates $z$ from $A$, so $x=\mathfrak{g}_{A}(z)$. It remains to show that $z \in A^{\prime}$, but this follows from Lemma 8.6 .

Lemma 8.6. Let $\mathcal{X}$ be a $C A T(0)$ cube complex and let $\mathcal{Y}, \mathcal{Z}$ be convex subcomplexes, with $A=\mathcal{Y} \cap \mathcal{Z}$. Then for all $x \in \mathcal{Z}$, we have $\mathfrak{g}_{\mathcal{Y}}(x) \in A$.

Proof. Let $y=\mathfrak{g}_{\mathcal{Y}}(x)$, let $a=\mathfrak{g}_{A}(x)$, and let $m$ be the median of $x, a$, and $z$. Then $m \in \mathcal{Z}$ since it lies on a geodesic from $x$ to $a$, and $x, a \in \mathcal{Z}$, and $\mathcal{Z}$ is convex. Similarly, $m$ lies on a geodesic from $y$ to $a$, and thus $m \in \mathcal{Y}$. It follows that $m \in A$, whence $m=a$ since $\mathrm{d}_{\mathcal{X}}(x, m) \leqslant \mathrm{d}_{\mathcal{X}}(x, a)$. But then $\mathrm{d}_{\mathcal{X}}(x, a) \leqslant \mathrm{d}_{\mathcal{X}}(x, y)$, so $y=a$.

If $C$ is a special cube complex then $C$ admits a local isometry into some Salvetti complex by [HW08, Theorem 1.1], and this is the Salvetti complex of a finitely-generated right-angled Artin group when $C$ has finitely many immersed hyperplanes (e.g., when $C$ is compact special). Such a local isometry lifts to a convex embedding at the level of universal covers, whence Proposition 8.3 and Lemma 8.5 immediately imply that the universal cover of $C$ admits a factor system. (Note that finite generation of the right-angled Artin group is important, since otherwise the universal cover of the Salvetti complex does not have a factor system because each 0 -cube is contained in infinitely many combinatorial hyperplanes in this case.) We will now describe such factor systems.

Definition 8.7. Let $C$ be a special cube complex whose set $\mathcal{H}$ of immersed hyperplanes is finite. For every $\mathcal{A} \subseteq \mathcal{H}$ and 1-cubes $e, e^{\prime}$ of $C$, write $e \sim_{\mathcal{A}} e^{\prime}$ if there is a path $e=e_{0} \ldots e_{n}=e^{\prime}$ in the 1-skeleton of $C$ so that each $e_{i}$ is dual to some immersed hyperplane from $\mathcal{A}$. Let $C_{\mathcal{A}}$ be the collection of full subcomplexes of $C$ whose 1-skeleton is an equivalence class of 1-cubes with respect to the equivalence relation $\sim \mathcal{A}$. 
Notice that each $D \in C_{\mathcal{A}}$ is locally convex.

Corollary 8.8 (Factor systems for special groups). Let $\mathcal{X}$ be the universal cover of a special cube complex, $C$, with finitely many immersed hyperplanes, $\mathcal{H}$. Then $\mathcal{X}$ admits a factor system: the collection of all lifts of subcomplexes in $\bigcup_{\mathcal{A} \subseteq \mathcal{H}} C_{\mathcal{A}}$ is a factor system for $\mathcal{X}$.

Proof. Let $\Gamma$ be the crossing graph of $C$, which has a vertex for each immersed hyperplane, with two vertices adjacent if the corresponding immersed hyperplanes have nonempty intersection. Then for each immersed hyperplane $H$ of $C$ there is a corresponding 1-cube $e_{H}$ in $S_{\Gamma}$. In [HW08, Theorem 1.1] it is shown that there is a local isometry $\phi: C \rightarrow S_{\Gamma}$ so that if the 1-cube $e$ is dual to the immersed hyperplane $H$ then it gets mapped isometrically to $e_{H}$.

The local isometry $\phi$ lifts to a convex embedding $\tilde{\phi}: \mathcal{X} \rightarrow \widetilde{S}_{\Gamma}$ (see e.g. [Wis11, Lemma 3.12]), so in view of Proposition 8.3 and Lemma 8.5 there is a factor system on $\mathcal{X}$ consisting of all preimages of elements of the factor system $\mathfrak{F}$ for $\widetilde{S}_{\Gamma}$, where $\mathfrak{F}$ is the factor system associated to the collection of all subgraphs of $\Gamma$ described in Proposition 8.3 .

From now on we identify the 0 -skeleton of $\widetilde{S}_{\Gamma}$ with $A(\Gamma)$, and regard the 1-cubes of $\widetilde{S}_{\Gamma}$ as labeled by an immersed hyperplane of $C$ (they are naturally labeled by vertices of $\Gamma$, which are immersed hyperplanes of $C$ ).

Let us consider an element of $F \in \mathfrak{F}$ that intersects $\tilde{\phi}(\mathcal{X})$ in, say, $g \in A(\Gamma)$. We want to show that $F^{\prime}=F \cap \tilde{\phi}(\mathcal{X})$ is the image via $\tilde{\phi}$ of the lift $\widetilde{D}$ of some $D \in \bigcup C_{\mathcal{A}}$.

The 0 -skeleton of $F$ is $g A(\Lambda)$ for some subgraph $\Lambda$ of $\Gamma$ whose set of vertices will be denoted $\mathcal{A}$. By convexity of $F^{\prime}$ we know that we can connect $g$ to any element of the 0 -skeleton of $F^{\prime}$ (that is to say $g A(\Lambda) \cap \tilde{\phi}(\mathcal{X})$ ) by a path in the 1 -skeleton of $F^{\prime}$ all whose 1 -cubes are labeled by elements of $\mathcal{A}$. On the other hand, if we can connect $g$ to, say, $h$ by a path in the 1-skeleton of $F^{\prime}$ all whose 1-cubes are labeled by elements of $\mathcal{A}$, then $h \in g A(\Lambda)$. These facts easily imply that the 0 -skeleton of $F^{\prime}$ coincides with the 0-skeleton of $\tilde{\phi}(\widetilde{D})$ for some $D \in C_{\mathcal{A}}$, which in turn implies $\tilde{\phi}(\widetilde{D})=F \cap \tilde{\phi}(\mathcal{X})$.

It can be similarly shown that for any $D \in \bigcup_{\mathcal{A} \subseteq \mathcal{H}} C_{\mathcal{A}}$, we have that $\phi(\widetilde{D})$ is of the form $F \cap \tilde{\phi}(\mathcal{X})$ for some $F \in \mathfrak{F}$, and this concludes the proof.

The same proof goes through to show the following more general version of the corollary:

Corollary 8.9. Let $\mathcal{X}$ be the universal cover of the special cube complex $C$, whose set of immersed hyperplanes is finite, and let $\Gamma$ be the crossing graph of the immersed hyperplanes of $C$. If $\mathcal{R}$ is a rich collection of subgraphs of $\Gamma$, then the collection of all lifts of subcomplexes in $\bigcup_{\Lambda \in \mathcal{R}} C_{\Lambda^{(0)}}$ is a factor system for $\mathcal{X}$.

\subsubsection{A non-special example.}

Example 8.10. Let $S, T$ be wedges of finitely many circles and let $D$ be a compact, 2dimensional, nonpositively-curved cube complex with the following properties:

(1) $D^{(1)}=S \cup T$.

(2) The universal cover $\widetilde{D}$ of $D$ is $\widetilde{S} \times \widetilde{T}$.

(3) $H=\pi_{1} D$ has no nontrivial finite quotient.

Such $D$ were constructed by Burger-Mozes [BM00, whose complex actually has simple fundamental group, and by Wise [Wis07]. Let $\tilde{\alpha} \rightarrow \widetilde{S}$ and $\tilde{\beta} \rightarrow \widetilde{T}$ be nontrivial combinatorial geodesics mapping to immersed closed combinatorial paths $\alpha, \beta \rightarrow D$. Since each of $\widetilde{S}, \widetilde{T}$ is convex in $\widetilde{S} \times \widetilde{T}$, each of the maps $\alpha \rightarrow D, \beta \rightarrow D$ is a local isometry of cube complexes. Hence $[\alpha],[\beta] \in H-\{1\}$ respectively lie in the stabilizers of the hyperplanes $\widetilde{S} \times\{o\},\left\{o^{\prime}\right\} \times \widetilde{T}$ of $\widetilde{S} \times \widetilde{T}$, where $\left(o^{\prime}, o\right)$ is a basepoint chosen so that $o^{\prime}, o$ are arbitrary midpoints of 1 -cubes of $\widetilde{S}, \widetilde{T}$. Suppose moreover that $|\alpha|=|\beta|=r \geqslant 1$. 
Let $R=[0, r] \times[0,2]$. Regard $R$ as a 2-dimensional non-positively-curved cube complex in the obvious way, and form a complex $Z_{0}$ from $R \sqcup D$ by identifying $\{0\} \times[0,2]$ with $\{r\} \times[0,2]$ and identifying the images of $[0, r] \times\{0\}$ and $[0, r] \times\{2\}$ with $\alpha$ and $\beta$ respectively. Since $\alpha$ and $\beta$ are locally convex in both $R$ and $D$, the complex $Z_{0}$ is nonpositively-curved, and its fundamental group is $H *\langle[\alpha]\rangle t=\langle[\beta]\rangle$.

Let $A=[0,1] \times[0, r]$ with the obvious cubical structure, and attach $A$ to $Z_{0}$ by identifying $\{0\} \times[0, r]$ with the "meridian" in $R$, i.e., the image of $[0, r] \times\{1\}$. Finally, attach a square by gluing a length-2 subpath of its boundary path to the image of $[0,1] \times\{0\} \cup[0,1] \times\{r\} \subset A$ in $A \cup Z_{0}$. The resulting complex is $Z$. Since $C$ is locally convex in $Z_{0}$ and $\{0\} \times[0, r]$ is locally convex in $A$, the complex $Z$ is a nonpositively-curved cube complex. See Figure 3 .

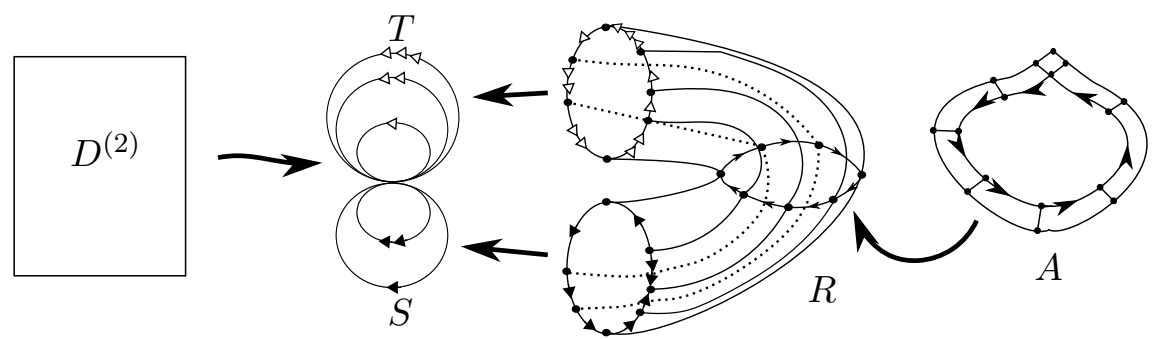

Figure 3. A complex $Z$ with $r=7$. The square at left represents the 2skeleton of $D$. Note that $A$ is the carrier of a self-intersectign hyperplane, $A$ is attached to $R$ by identifying the "inner" boundary component with the waist of $R$, and $R$ is attached to $S \cup T$ by a local isometry of its boundary.

The following properties of $Z$ will be needed in Section 11 .

Proposition 8.11. The universal cover $\tilde{Z}$ of $Z$ has a factor system.

Proof. Let $Z^{\prime}$ be constructed exactly as in the construction of $Z$ in Example 8.10, except beginning with $S \times T$ instead of with the complex $D$. The 1-skeleta of $D$ and $S \times T$ are identical, so the paths $\alpha, \beta$ exist in $S \times T$, rendering this construction possible. It is easily verified that the universal cover of $Z^{\prime}$ is isomorphic to $\widetilde{Z}$ and that $Z^{\prime}$ has a finite-sheeted special cover. The claim follows from Corollary 8.8 .

Proposition 8.12. Let $G \leqslant f . i . \pi_{1} Z$. Then there exists a hyperplane $H$ of $\widetilde{D}$ and $g \in G$ such that $g H$ crosses $H$.

Proof. Let $\bar{W} \rightarrow Z$ be the immersed hyperplane dual to the image of $[0,1] \times\{0\} \subset A$ in $Z$. Then $\bar{W}$ self-crosses. Moreover, if $\widehat{Z} \rightarrow Z$ is a finite-sheeted cover such that some component $\widehat{C}$ of the preimage of the meridian $C$ has the property that $\widehat{C} \rightarrow C$ is bijective, then some lift of $W$ to $\widehat{Z}$ is again a self-crossing immersed hyperplane. But $C$ is homotopic into $D$, whose fundamental group has no finite quotients. Hence any finite cover of $Z$ has a self-crossing immersed hyperplane.

Having shown that there are many interesting groups with factor systems, below we show the utility of these systems. First though, we propose the following question to which we expect a positive answer; we believe that a proof of this will require significant work.

Question 8.13. Does every CAT(0) cube complex which admits a proper, cocompact group action contain a factor system? 


\subsection{Factored contact graphs.}

Definition 8.14 (Factored contact graphs, projection to the factored contact graph). Let $\mathfrak{F}$ be a factor system for $\mathcal{X}$. For each convex subcomplex $F \subseteq \mathcal{X}$, the factored contact graph $\widehat{\mathcal{C}} F$ is obtained from $\mathcal{C} F$ as follows.

Let $F^{\prime} \in \mathfrak{F}_{F}-\{F\}$ and suppose that $F^{\prime}$ is either parallel to a combinatorial hyperplane or has diameter $\geqslant \xi$, and let $\left[F^{\prime}\right]$ be its parallelism class. We add a vertex $v_{F^{\prime}}$ to $\mathcal{C} F$, corresponding to this parallelism class $\left[F^{\prime}\right]$, and join $v_{F^{\prime}}$ by an edge to each vertex of $\mathcal{C} F^{\prime} \subset$ $\mathcal{C} F$, i.e., each newly added vertex $v_{F^{\prime}}$ gets connected to each vertex of $\mathcal{C} F$ which corresponds to a hyperplane of $F$ that intersect $F^{\prime}$. We repeat this for each such $F^{\prime} \in \mathfrak{F}_{F}-\{F\}$. We emphasize that $\widehat{\mathcal{C}} F$ consists of $\mathcal{C} F$, together with a cone-vertex for each parallelism class of subcomplexes in $\mathfrak{F}_{F}-\{F\}$ that are parallel to combinatorial hyperplanes or have diameter $\geqslant \xi$ (or both). Also, observe that $\mathcal{C} F$ is an induced subgraph of $\widehat{\mathcal{C}} F$.

The projection of $\mathcal{X}$ to $\widehat{\mathcal{C}} F$ is the map $\pi_{F}: \mathcal{X} \rightarrow 2^{\widehat{\mathcal{C}} F^{(0)}}$ obtained by composing the projection to $\mathcal{C} F$ given in Definition 4.1 with the inclusion $\mathcal{C} F \hookrightarrow \widehat{\mathcal{C}} F$. Recall that this map assigns to each point of $\mathcal{X}$ a clique in $\widehat{\mathcal{C} F}$ consisting of the vertices corresponding to the hyperplanes whose carriers contain the given point.

Remark 8.15. When $F \in \mathfrak{F}$ is a single 0 -cube, $\mathcal{C} F=\varnothing$, so $\widehat{\mathcal{C}} F=\varnothing$. Hence $\left|2^{\widehat{\mathcal{C}} F^{(0)}}\right|=1$ and $\pi_{F}$ is defined in the obvious way. When the constant $\xi$ from Definition 8.1 is at least 1 , no $F \in \mathfrak{F}$ is a single point.

Lemma 8.16. Let $F, F^{\prime}$ be parallel. Then there is a bijection $f: \mathfrak{F}_{F} \rightarrow \mathfrak{F}_{F^{\prime}}$ so that $f(H)$ is parallel to $H$ for all $H \in \mathfrak{F}_{F}$.

Proof. If $F^{\prime \prime} \in \mathfrak{F}$ is contained in $F$, then there is a parallel copy $F^{\prime \prime \prime}$ of $F^{\prime \prime}$ in $F^{\prime}$. Provided either $F^{\prime \prime}$ is a combinatorial hyperplane or has diameter at least $\xi$, we have $F^{\prime \prime \prime} \in \mathfrak{F}$. The existence of $f$ now follows easily.

The next lemma is immediate.

Lemma 8.17. Let $F \in \mathfrak{F}$ and let $F^{\prime}$ be a convex subcomplex parallel to $F$. Then, $\mathcal{C} F=\mathcal{C} F^{\prime}$ and the bijection $f: \mathfrak{F}_{F} \rightarrow \mathfrak{F}_{F^{\prime}}$ from Lemma 8.16 descends to a bijection of parallelism classes inducing an isomorphism $\widehat{\mathcal{C}} F \rightarrow \widehat{\mathcal{C}} F^{\prime}$ that extends the identity $\mathcal{C} F \rightarrow \mathcal{C} F^{\prime}$. Moreover, $\pi_{F}=\pi_{F^{\prime}}$.

Remark 8.18 (Crossing, osculation, and coning). In the case where $\mathfrak{F}$ is the factor system obtained by closing the set of subcomplexes parallel to hyperplanes under diameter- $\geqslant \xi$ projections, then $\hat{\mathcal{C}} \mathcal{X}$ is quasi-isometric to $\mathcal{C X}$. This is because we are coning off contact graphs of intersections of hyperplane-carriers, and these subgraphs are already contained in vertex-links in $\mathcal{C} \mathcal{X}$. More generally, let $H_{I}=\cap_{i \in I} H_{i}^{+}$be an intersection of combinatorial hyperplanes. The hyperplanes of $H_{I}$ have the form $W \cap H_{I}$, where $W$ is a hyperplane that crosses each of the hyperplanes $H_{i}$. Coning off $\mathcal{C}\left(W^{ \pm} \cap H_{I}\right)$ in $\mathcal{C} H_{I}$ does not affect the quasiisometry type, since $\mathcal{C}\left(W^{ \pm} \cap H_{I}\right)$ is the link of a vertex of $\mathcal{C} H_{I}$. It is when $W$ is disjoint from $H_{I}$ but exactly one of $W^{ \pm}$is not - i.e., precisely when $W$ osculates with each $H_{i}$ that coning off $\mathcal{C}\left(W^{+} \cap H_{I}\right) \subset \mathcal{C} H_{I}$ affects the geometry.

The crucial property of projections to factored contact graphs is:

Lemma 8.19 (Bounded projections). Let $\mathfrak{F}$ be a factor system for $\mathcal{X}$ and let $\xi$ be the constant from Definition 8.1. Let $F, F^{\prime} \in \mathfrak{F}$. Then one of the following holds:

(1) $\operatorname{diam}_{\widehat{\mathcal{C}} F}\left(\pi_{F}\left(F^{\prime}\right)\right)<\xi+2$.

(2) $F$ is parallel to a subcomplex of $F^{\prime}$. 
Proof. If $\operatorname{diam}\left(\mathfrak{g}_{F}\left(F^{\prime}\right)\right) \geqslant \xi$, then either $\pi_{F}\left(F^{\prime}\right)$ is coned off in $\widehat{\mathcal{C}} F$ or $\mathfrak{g}_{F}\left(F^{\prime}\right)=F$.

We also note the following version of Proposition 4.2 for factored contact graphs:

Proposition 8.20 (Bounded Geodesic Image II: The Factoring). Let $F \in \mathfrak{F}$ and let $\gamma \rightarrow F$ be a combinatorial geodesic. Suppose $U \in \mathfrak{F}_{F}$ and that $\mathrm{d}_{\widehat{\mathcal{C}} F}\left(\pi_{F}(\gamma), \widehat{\mathcal{C}} U\right) \geqslant 1$. Then $\mathfrak{g}_{U}(\gamma)$ consists of a single $0-$ cube. Hence $\pi_{U}(\gamma)$ is a clique.

Proof. Suppose that $\mathfrak{g}_{U}(\gamma)$ contains distinct 0-cubes $x, y$ and let $H$ be a hyperplane separating them. Then $H$ crosses $U$ and thus corresponds to a vertex of $\mathcal{C} U \subset \widehat{\mathcal{C}} U \subseteq \widehat{\mathcal{C}} F$. On the other hand, $H$ cannot separate any 0 -cube of $\gamma$ from $U$ and thus separates the endpoints of $\gamma$. Hence $H$ is a vertex of $\pi_{F}(\gamma)$.

8.4. Hierarchy paths revisited. Let $\mathfrak{F}$ be a factor system for $\mathcal{X}$ and let $F \in \mathfrak{F}$. The vertices of $\hat{\mathcal{C}} F$ are naturally associated to subcomplexes of $\mathcal{X}$ : to each vertex $V$ of $\mathcal{C} F$ $(\subset \widehat{\mathcal{C}} F)$, we associate one of the two combinatorial hyperplanes $V^{ \pm}$bounding $\mathcal{N}(V)$. The remaining vertices are cone points corresponding to parallelism classes of subcomplexes in $\mathfrak{F}_{F}$. A path of $\widehat{\mathcal{C}} F$ is a sequence $\left(v_{0}, \ldots, v_{n}\right)$ of vertices with consecutive vertices adjacent. A combinatorial path $\gamma \rightarrow F$ is carried by the path $\left(v_{0}, \ldots, v_{n}\right)$ of $\widehat{\mathcal{C}} F$ if $\gamma=\gamma_{0} e_{0} \gamma_{1} e_{1} \cdots \gamma_{n} e_{n}$, where for $0 \leqslant i \leqslant n$, we have $\gamma_{i} \rightarrow T_{i}$ with $T_{i}$ associated to $v_{i}$ and $\left|e_{i}\right| \leqslant 1$. In this situation, we say that the sequence $T_{0}, \ldots, T_{n}$ carries $\gamma$ and represents $\left(v_{0}, \ldots, v_{n}\right)$.

Proposition 8.21. Let $v_{0}, v_{1}, \ldots, v_{r}$ be a path in $\widehat{\mathcal{C}} \mathcal{X}$ and let $x \in T_{0}, y \in T_{r}$ be 0 -cubes, where $T_{0}, T_{r}$ are subcomplexes associated to $v_{0}, v_{r}$. Then for $1 \leqslant i \leqslant r-1$, we can choose for each vertex $v_{i}$ an associated subcomplex $T_{i}$ so that $T_{0}, \ldots, T_{r}$ carries a path in $\mathcal{X}$ joining $x$ to $y$.

Proof. We argue by induction on $r$. If $r=0$, then the claim follows by path-connectedness of $T_{0}$. Now let $r \geqslant 1$. Suppose first that $v_{r}$ is a cone-vertex, so that $v_{r-1}$ is a hyperplanevertex, and let $V$ be the corresponding hyperplane. Since $V$ crosses $T_{r}$, either combinatorial hyperplane $T_{r-1}$ bounding the carrier of $V$ intersects $T_{r}$. There exists a 0 -cube $y^{\prime} \in T_{r-1} \cap$ $T_{r}$. On the other hand, if $v_{r}$ is a hyperplane-vertex, then $T_{r}$ is a specified combinatorial hyperplane parallel to a hyperplane crossing each subcomplex $T_{r-1}$ associated to $v_{r-1}$, if $v_{r-1}$ is a cone-vertex. If $v_{r-1}$ is also a hyperplane-vertex, then at least one of the possible choices of $T_{r-1}$ is a combinatorial hyperplane intersecting the combinatorial hyperplane $T_{r}$. Again, we have a 0 -cube $y^{\prime} \in T_{r} \cap T_{r-1}$. In either case, by induction, we can choose $T_{1}, \ldots, T_{r-2}$ to carry a path joining $x$ to $y^{\prime}$, which we concatenate with a path in $T_{r}$ joining $y^{\prime}$ to $y$. The path $e_{r-2}$ is nontrivial if $v_{r-1}$ corresponds to a hyperplane separating $T_{r}$ from every possible choice of $T_{r-2}$.

Remark 8.22 (Explicit description of paths carried by geodesics). In the proof of Proposition 8.21, we used the fact that a path $v_{0}, v_{1}, \ldots, v_{r}$ in $\widehat{\mathcal{C}} F$ has the property that, if $v_{i}$ is a cone-vertex, associated to some $U \in \mathfrak{F}_{F}$, then $v_{i \pm 1}$ are hyperplane vertices, associated to hyperplanes $H_{i \pm 1}$ that cross $U$, so that all four combinatorial hyperplanes $H_{i \pm 1}^{ \pm}$intersect $U$. Hence we can and shall always assume that $\left|e_{i \pm 1}\right|=0$ when $v_{i}$ is a cone-vertex, see Figure 4 . This enables us to use the proof of Proposition 3.1 verbatim in the proof of Proposition 8.23

In view of the previous proposition, we will use the same notation for a vertex of $\widehat{\mathcal{C}} F$ as for some representative subcomplex. A hierarchy path is a geodesic of $F$ that is carried by a geodesic of $\hat{\mathcal{C}} F$.

Proposition 8.23. Let $F \in \mathfrak{F}$. Then any 0 -cubes $x, y \in F$ are joined by a hierarchy path.

Proof. Let $\tau$ be a combinatorial geodesic of $\mathcal{X}$ joining $x$ to $y$; since $F$ is convex, $\tau \subseteq F$. Let $T \in \mathfrak{F}_{F}$ contain $x$ (such $T$ exists because every 0 -cube of $F$ is contained in a combinatorial 


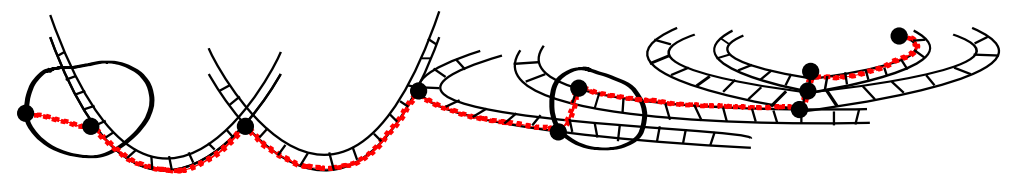

FiguRE 4. The two round regions are elements of $\mathfrak{F}$; the ladders represent carriers of hyperplanes. The dotted path is carried by a geodesic in the factored contact graph. Transitions between various $\gamma_{i}$ and $e_{i}$ are indicated by bold vertices. Notice that there are numerous such paths, but we choose paths with $e_{i}$ trivial whenever possible.

hyperplane of $F$ ) and let $T^{\prime} \in \mathfrak{F}_{F}$ contain $y$. Let $T=T_{0}, T_{1}, \ldots, T_{n}=T^{\prime}$ be a geodesic of $\widehat{\mathcal{C}} F$. Then we can choose the $T_{i}$ in their parallelism classes so that $\mathcal{N}_{1}\left(T_{i}\right) \cap \mathcal{N}_{1}\left(T_{i+1}\right) \neq \varnothing$ for $0 \leqslant i \leqslant n-1$. Using Proposition 8.21 , for each $i$, let $\gamma_{i} \rightarrow T_{i}$ be a geodesic segment, chosen so that $\gamma_{1} e_{1} \ldots \gamma_{n} e_{n}$ is a piecewise-geodesic path joining $x$ to $y$, with each $\left|e_{i}\right| \leqslant 1$ and $\left|e_{i}\right|=0$ except possibly if $\gamma_{i}, \gamma_{i+1}$ lie in disjoint combinatorial hyperplanes representing vertices of $\mathcal{C} F$ (i.e., non-cone-vertices). The disc diagram argument from the proof of Proposition 3.1 can now be repeated verbatim to complete the proof, because $\mathfrak{F}_{F}$ satisfies property (4) from Definition 8.1.

8.5. Factored contact graphs are quasi-trees. In this section, we will use the following criterion of Manning, that determines if a geodesic metric space is quasi-isometric to a tree:

Proposition 8.24 (Bottleneck criterion; Man05]). Let $(Z, d)$ be a geodesic metric space. Suppose that there exists $\delta \geqslant 0$ such that for all $x, y \in Z$, there exists $m=m(x, y)$ such that $d(x, m)=d(y, m)=\frac{1}{2} d(x, y)$ and every path joining $x$ to $y$ intersects the closed $\delta$-ball about $m$. Then $(Z, d)$ is $(26 \delta, 16 \delta)$-quasi-isometric to a tree.

Proposition 8.25. Let $F \in \mathfrak{F}$. Then $\widehat{\mathcal{C}} F$ is $(78,48)$-quasi-isometric to a simplicial tree.

Proof. Denote by $\mathcal{H}$ the set of vertices of $\widehat{\mathcal{C}} F$ corresponding to hyperplanes of $F$ and by $\mathcal{V}$ the set of cone-vertices. Let $T, T^{\prime}$ be complexes associated to vertices of $\widehat{\mathcal{C}} F$ (regarded as representatives, to be chosen, of parallelism classes of subcomplexes in $\mathfrak{F}_{F}$ ). Let $T=$ $T_{0}, T_{1}, \ldots, T_{n}=T^{\prime}$ be a geodesic of $\widehat{\mathcal{C}} F$ joining $T$ to $T^{\prime}$ and let $m$ be the midpoint of this geodesic. Suppose, moreover, that $T_{0}, \ldots, T_{n}$ carries a hierarchy path $\gamma=\gamma_{0} \cdots \gamma_{n}$ such that each hyperplane intersecting $\gamma$ separates $T$ from $T^{\prime}$; indeed we can choose $\gamma$ to connect a point $p \in \mathfrak{g}_{T}\left(T^{\prime}\right)$ to its gate $\mathfrak{g}_{T^{\prime}}(p)$. We may assume that $n \geqslant 4$, for otherwise Proposition 8.24 is satisfied by $m$ with $\delta=\frac{3}{2}$. Hence, since every vertex adjacent to an element of $\mathcal{V}$ is in $\mathcal{H}$, there exists $i$ so that $T_{i}$ is a combinatorial hyperplane and $\mathrm{d}_{\widehat{\mathcal{C}} F}\left(T_{i}, m\right) \leqslant 1$. Let $T_{i}^{\prime}$ be the hyperplane such that $T_{i}$ is in the image of $T_{i}^{\prime} \times\{ \pm 1\}$ under $T_{i}^{\prime} \times[-1,1] \cong \mathcal{N}\left(T_{i}^{\prime}\right) \hookrightarrow F$. Since every hyperplane crossing $\gamma_{i}$ separates $T$ from $T^{\prime}$, we have that either $T_{i}^{\prime}$ separates $T$ from $T^{\prime}$, or $T_{i}^{\prime}$ crosses a hyperplane that separates $T$ from $T^{\prime}$. Hence, there is a hyperplane $H$ such that $H$ separates $T, T^{\prime}$ and $\mathrm{d}_{\widehat{\mathcal{C}} F}(H, m) \leqslant 2$. Let $T=S_{0}, \ldots, S_{k}=T^{\prime}$ be another path in $\widehat{\mathcal{C}} F$ joining $T$ to $T^{\prime}$. Then, there exists $j$ such that either $S_{j}$ is a combinatorial copy of $H$, or $H$ crosses $S_{j}$. Thus $\mathrm{d}_{\widehat{\mathcal{C}} F}\left(m, S_{j}\right) \leqslant 3$, and the claim follows from Proposition 8.24 .

\section{The DistanCE FORMUla}

The main theorem of this section is:

Theorem 9.1 (Distance formula). Let $\mathcal{X}$ be a CAT(0) cube complex and let $\mathfrak{F}$ be a factor system. Let $\overline{\mathfrak{F}}$ contain exactly one representative of each parallelism class in $\mathfrak{F}$. Then there 
exists $s_{0} \geqslant 0$ such that for all $s \geqslant s_{0}$, there are constants $K \geqslant 1, C \geqslant 0$ such that for all $x, y \in \mathcal{X}^{(0)}$,

$$
\mathrm{d}_{\mathcal{X}}(x, y) \asymp_{K, C} \sum_{F \in \overline{\mathfrak{F}}}\left\{\mathrm{d}_{\widehat{\mathcal{C}} F}\left(\pi_{F}(x), \pi_{F}(y)\right)\right\}_{s} .
$$

The rest of this section is devoted to proving Theorem 9.1. Throughout, $\mathcal{X}$ and $\mathfrak{F}$ are as in the statement of the theorem. The constants $\xi \geqslant 1, \Delta \geqslant 1$ are those from Definition 8.1. For convenience, if $F \in \mathfrak{F}$ and $x, y \in \mathcal{X}$, we write $\mathrm{d}_{\widehat{\mathcal{C}} F}(x, y):=\mathrm{d}_{\widehat{\mathcal{C}} F}\left(\pi_{F}(x), \pi_{F}(y)\right)$.

\subsection{Projection lemmas.}

Lemma 9.2. Let $\mathcal{X}$ be a $C A T(0)$ cube complex, let $A \subseteq B \subseteq \mathcal{X}$ be convex subcomplexes, and let $x, y \in \mathcal{X}$ be 0-cubes. Then $\mathrm{d}_{\widehat{\mathcal{C}} A}\left(\pi_{A}(x), \pi_{A}(y)\right) \leqslant \mathrm{d}_{\mathcal{X}}\left(\mathfrak{g}_{A}(x), \mathfrak{g}_{A}(y)\right) \leqslant \mathrm{d}_{\mathcal{X}}\left(\mathfrak{g}_{B}(x), \mathfrak{g}_{B}(y)\right)$.

Proof. The first inequality follows since projection to the factored contact graph is distance non-increasing. The second inequality is Lemma 2.1.

Lemma 9.3. Let $x, y \in \mathcal{X}^{(0)}$ and let $T_{0}, T_{1}, \ldots, T_{r}$ represent a geodesic in $\widehat{\mathcal{C}} \mathcal{X}$ with $x \in T_{0}, y \in$ $T_{r}$. For $0 \leqslant i \leqslant r$, let $x_{i}=\mathfrak{g}_{T_{i}}(x), y_{i}=\mathfrak{g}_{T_{i}}(y)$. Then

$$
\mathrm{d}_{\mathcal{X}}(x, y)-\mathrm{d}_{\hat{\mathcal{C}} \mathcal{X}}(x, y) \leqslant \sum_{i=0}^{r} \mathrm{~d}_{T_{i}}\left(x_{i}, y_{i}\right) \leqslant 3 \mathrm{~d}_{\mathcal{X}}(x, y) .
$$

Proof. For $0 \leqslant i \leqslant r$, let $\mathcal{S}_{i}$ be the set of hyperplanes separating $x_{i}, y_{i}$, so that $\left|\mathcal{S}_{i}\right|=$ $\mathrm{d}_{\mathcal{X}}\left(x_{i}, y_{i}\right)=\mathrm{d}_{T_{i}}\left(x_{i}, y_{i}\right)$. For each $i$, observe that if the hyperplane $U$ separates $x$ from $y$ and crosses $T_{i}$, then $U$ separates $x_{i}$ from $y_{i}$. Otherwise, $U$ would separate $x_{i}$ from $x$ (say), but the only such hyperplanes are those separating $x$ from $T_{i}$, so this situation would contradict the fact that $U$ crosses $T_{i}$. Hence the set of hyperplanes separating $x$ from $y$ and crossing some $T_{i}$ is $\cup_{i=0}^{r} \mathcal{S}_{i}$. Each hyperplane $H$ separating $x, y$ either crosses some $T_{i}$, or there exists $i$ so that $\mathcal{N}(H)$ contains $T_{i}$ in one of the bounding copies of $H$. Hence

$$
\mathrm{d}_{\mathcal{X}}(x, y) \leqslant\left|\cup_{i=0}^{r} \mathcal{S}_{i}\right|+r \leqslant \sum_{i=0}^{r}\left|\mathcal{S}_{i}\right|+r,
$$

which establishes the first inequality.

For some $i \neq j$, let $U \in \mathcal{S}_{i} \cap \mathcal{S}_{j}$. Then $|i-j| \leqslant 2$, since otherwise $T_{i}, U, T_{j}$ would provide a shortcut contradicting the fact that $\mathrm{d}_{\widehat{\mathcal{C}}(\mathcal{X}, \mathcal{P})}\left(T_{0}, T_{r}\right)=r$. Hence at most three elements of $\left\{\mathcal{S}_{i}\right\}_{i=0}^{r}$ contain $U$. Thus $\sum_{i=0}^{r}\left|\mathcal{S}_{i}\right| \leqslant 3\left|\cup_{i=0}^{r} \mathcal{S}_{i}\right|$, and the second inequality follows.

Proposition 9.4 (Large Link Lemma). Let $T_{0}, T_{1}, \ldots, T_{r}$ be a geodesic in $\hat{\mathcal{C}} \mathcal{X}$ between 0 cubes $x \in T_{0}$ and $y \in T_{r}$. Let $F \in \mathfrak{F}-\{\mathcal{X}\}$ have the property that $\mathrm{d}_{\widehat{\mathcal{C}} F}(x, y) \geqslant 4 \xi+10$. Then there exists $i \in\{0,1, \ldots, r\}$ such that $F$ is parallel to some $F^{\prime} \in \mathfrak{F}_{T_{i}}$. Moreover, any geodesic of $\mathcal{X}$ contained in $\cup_{i} T_{i}$ that joins $x, y$ passes through a subcomplex of $T_{i}$ parallel to $F^{\prime}$.

Proof. Recall that we can choose $T_{i}$ within its parallelism class for $1 \leqslant i \leqslant r-1$ so that $\mathcal{N}_{1}\left(T_{i}\right) \cap T_{i+1} \neq \varnothing$. We first exhibit $J \leqslant 3$ and $i \leqslant r$ such that for some $j$ satisfying $0 \leqslant j \leqslant J$ we have $\operatorname{diam}_{\widehat{\mathcal{C}} F}\left(\pi_{F}\left(T_{i+j}\right)\right) \geqslant \xi$ and for all $i^{\prime}$ satisfying $i^{\prime}<i$ or $i^{\prime}>i+J$ we have $\operatorname{diam}_{\widehat{\mathcal{C}} F}\left(\pi_{F}\left(T_{i^{\prime}}\right)\right)=0$.

Let $V$ be a hyperplane separating $\mathfrak{g}_{F}(x)$ from $\mathfrak{g}_{F}(y)$. Then $V$ intersects $F$ and hence $V$ cannot separate $x$ or $y$ from their gates in $F$. Hence $V$ must separate $x$ from $y$; and thus $V$ intersects some $\mathcal{N}_{1}\left(T_{i}\right)$. Either $V$ separates $\mathfrak{g}_{T_{i}}(x)$ from $\mathfrak{g}_{T_{i}}(y)$, or $V$ is the unique hyperplane separating $T_{i}$ from $T_{i \pm 1}$. Let $\mathcal{G}$ be the set of hyperplanes $V$ of the former type.

Let $V \in \mathcal{G}$. Then $V$ intersects $T_{i}$ for at least 1 and at most 3 values of $i$. If some other hyperplane $V^{\prime} \in \mathcal{G}$ separates $\mathfrak{g}_{F}(x)$ from $\mathfrak{g}_{F}(y)$, then $V^{\prime}$ separates $\mathfrak{g}_{T_{j}}(x)$ from $\mathfrak{g}_{T_{j}}(y)$ for at least 1 and at most 3 values of $j$, and for any such $i, j$, we have $|i-j| \leqslant 4$; otherwise 
$T_{i}, V, F, V^{\prime}, T_{j}$ would be a shortcut from $T_{i}$ to $T_{j}$, since $F \neq \mathcal{X}$ represents a vertex in $\hat{\mathcal{C}} \mathcal{X}$. Let $i, i+1, \ldots, i+J$ be the indices for which $T_{i+j}$ is crossed by a hyperplane separating $\mathfrak{g}_{F}(x)$ from $\mathfrak{g}_{F}(y)$; equivalently, the indices so that $\mathfrak{g}_{T_{k}}(F)$ is trivial if $k<i$ or $k>i+J$.

The graph $\pi_{F}\left(\cup_{j=0}^{J} \mathfrak{g}_{F}\left(T_{i+j}\right)\right)=\pi_{F}\left(\cup_{j=0}^{r} \mathfrak{g}_{F}\left(T_{i}\right)\right)$ has diameter at least $4 \xi+10$, since it contains $\pi_{F}(x), \pi_{F}(y)$, whence $\operatorname{diam}_{\widehat{\mathcal{C}} F}\left(\mathfrak{g}_{F}\left(T_{i+j}\right)\right) \geqslant \xi$ for some $j$. Indeed, there is at most one hyperplane separating $T_{i+j}$ from $T_{i+j+1}$, whence at most one hyperplane separates their gates in $F$. We then have that $F$ is parallel to a subcomplex of $T_{i+j}$ by Lemma 8.19.

Let $\gamma \subset \cup_{k} T_{k}$ be a geodesic joining $x, y$ and let $\gamma_{i+j}=\mathfrak{g}_{T_{i+j}}(\gamma)$. Then $\gamma_{i+j}$ has a subpath $\tau$ joining $\mathfrak{g}_{T_{i+j}}\left(\mathfrak{g}_{F}(x)\right)$ to $\mathfrak{g}_{T_{i+j}}\left(\mathfrak{g}_{F}(y)\right)$. The path $\tau$ is parallel to a path in $F$ and hence belongs to some parallel copy of $F$ in $T_{i+1}$.

Lemma 9.5. There exist $s_{0} \geqslant 0$ such that for all $x, y \in \mathcal{X}^{(0)}$ and any hierarchy path $\gamma_{0} e_{0} \cdots \gamma_{r} e_{r}$ joining $x$ to $y$ and carried by a geodesic $T_{0}, \ldots, T_{r}$ of $\widehat{\mathcal{C}} \mathcal{X}$, one of the following holds for all $F \in \mathfrak{F}$ :

(1) there exists $i \leqslant r$ and $F^{\prime} \in \mathfrak{F}_{T_{i}}$ such that $F$ is parallel to $F^{\prime}$;

(2) $\mathrm{d}_{\widehat{c} F}(x, y)<s_{0}$

(3) $F=\mathcal{X}$.

Proof. Let $s_{0}=4 \xi+10$ and let $F \neq \mathcal{X}$ be an element of $\mathfrak{F}$ with $\mathrm{d}_{\widehat{\mathcal{C}} F}(x, y) \geqslant s_{0}$. By Proposition 9.4 there exists $i$ so that $F$ is parallel to some $F^{\prime} \in \mathfrak{F}_{T_{i}}$, as required.

9.2. Proof of the distance formula. We have now assembled all ingredients needed for:

Proof of Theorem 9.1. For each $F \in \overline{\mathfrak{F}}$, let $\overline{\mathfrak{F}}_{F}$ consist of exactly one element from each parallelism class in $\mathfrak{F}_{F}$. We will argue by induction on $\Delta$, which we recall is the maximal number of elements in the factor system which can contain any given vertex.

Base case: When $\Delta=1$, the fact that $\mathcal{X}$ and each combinatorial hyperplane belongs to $\mathfrak{F}$ ensures that $\mathcal{X}$ consists of a single 0 -cube, so we are summing over the empty set of projections, and we are done.

Induction hypothesis: Assume $\Delta \geqslant 2$. For each $F \in \overline{\mathfrak{F}}-\{\mathcal{X}\}$, the set $\mathfrak{F}_{F}$ is a factor system in $F$. Every 0 -cube of $F$ is contained in at most $\Delta-1$ elements of $\mathfrak{F}_{F}$, since each 0 -cube of $F$ lies in at most $\Delta$ elements of $\mathfrak{F}$, one of which is $\mathcal{X}$ (which is not contained in $F$ ). We can therefore assume, by induction on $\Delta$, that for all $s \geqslant s_{0}$, where $s_{0} \geqslant 2$ is the constant from Lemma 9.5 there exist $K^{\prime} \geqslant 1, C^{\prime} \geqslant 0$ so that for all $F \in \overline{\mathfrak{F}}-\{\mathcal{X}\}$ and all $x, y \in \mathcal{X}$ we have

$$
\mathrm{d}_{F}\left(\mathfrak{g}_{F}(x), \mathfrak{g}_{F}(y)\right) \asymp_{K^{\prime}, C^{\prime}} \sum_{T \in \overline{\mathfrak{F}}_{F}}\left\{\left\{\mathrm{~d}_{\widehat{\mathcal{C}} T}\left(\mathfrak{g}_{F}(x), \mathfrak{g}_{F}(y)\right)\right\}_{s} .\right.
$$

Choosing a hierarchy path: By Proposition 8.23, there exists a hierarchy path $\gamma=$ $\gamma_{0} e_{0} \cdots \gamma_{r} e_{r}$ that joins $x$ to $y$ and is carried on a geodesic $T_{0}, \ldots, T_{r}$ in $\widehat{\mathcal{C}} \mathcal{X}$ from $T_{0}$ to $T_{r}$. For each $i$, let $x_{i}=\mathfrak{g}_{T_{i}}(x)$ and $y_{i}=\mathfrak{g}_{T_{i}}(y)$. Recall that each $\gamma_{i}$ lies in $\mathcal{N}_{1}\left(T_{i}\right)$. At the cost of adding 1 to our eventual multiplicative constant $K$, we can assume that each $e_{i}$ is trivial.

Enumeration of the nonvanishing terms: Let $s \geqslant s_{0}$ and let $F \in \mathfrak{F}$ be such that $\mathrm{d}_{\widehat{\mathcal{C}} F}(x, y) \geqslant s$. By Lemma 9.5, either $F$ is parallel to some $F^{\prime} \in \mathfrak{F}_{T_{i}}$ for some $i$, or $F=\mathcal{X}$.

Let $\mathfrak{F}_{\left\{T_{i}\right\}}$ be the set of all $F \in \mathfrak{F}$ that are parallel to a proper subcomplex of some $T_{i}$, and let $\overline{\mathfrak{F}}_{\left\{T_{i}\right\}}$ contain exactly one representative for each parallelism class of elements of $\mathfrak{F}_{\left\{T_{i}\right\}}$. 
Recalling that $\mathrm{d}_{\widehat{\mathcal{C}} F}(x, y)=\mathrm{d}_{\widehat{\mathcal{C}} F^{\prime}}(x, y)$ when $F$ is parallel to $F^{\prime}$, we get

$$
\sum_{F \in \widehat{\mathfrak{F}}}\left\{\left\{\mathrm{d}_{\widehat{\mathcal{C}} F}(x, y)\right\}_{s}=\mathbf{I}+\mathbf{I I}+\{r\}_{s}\right.
$$

where

$$
\mathbf{I}=\sum_{i=0}^{r}\left\{\left\{\mathrm{~d}_{\widehat{\mathcal{C}} T_{i}}(x, y)\right\}\right\}_{s}
$$

and

$$
\mathbf{I I}=\sum_{F \in \overline{\mathfrak{F}}_{\left\{T_{i}\right\}}}\left\{\left\{\mathrm{d}_{\widehat{\mathcal{C}} F}(x, y)\right\}_{s}=3,0 \sum_{i} \sum_{F \in \overline{\mathfrak{F}}_{T_{i}}-\left\{T_{i}\right\}}\left\{\left\{\mathrm{d}_{\widehat{\mathcal{C}} F}(x, y)\right\}_{s} .\right.\right.
$$

The estimate of II follows since no factor is parallel to both $T_{i}$ and $T_{i^{\prime}}$ when $\left|i-i^{\prime}\right| \geqslant 3$.

The upper bound: By the inductive hypothesis,

$$
\mathbf{I}+\mathbf{I I} \asymp_{K^{\prime}, C^{\prime}} \sum_{i=0}^{r} \mathrm{~d}_{T_{i}}\left(\mathfrak{g}_{T_{i}}(x), \mathfrak{g}_{T_{i}}(y)\right) .
$$

Hence, by Lemma 9.3 .

$$
\frac{\mathrm{d}_{\mathcal{X}}(x, y)-r}{3 K^{\prime}}-C^{\prime} \leqslant \mathbf{I}+\mathbf{I I}
$$

and we obtain the upper bound

$$
\begin{aligned}
\mathrm{d}_{\mathcal{X}}(x, y) & \leqslant 3 K^{\prime}(\mathbf{I}+\mathbf{I I}+r)+3 C^{\prime} K^{\prime} \\
& =3 K^{\prime} \sum_{F \in \overline{\mathfrak{F}}-\{\mathcal{X}\}}\left\{\left\{\mathrm{d}_{\widehat{\mathcal{C}} F}(x, y)\right\}_{s}+3 K^{\prime} \mathrm{d}_{\hat{\mathcal{C}} \mathcal{X}}(x, y)+3 C^{\prime} K^{\prime}\right. \\
& =_{1, s} \sum_{F \in \overline{\mathfrak{F}}-\{\mathcal{X}\}}\left\{\left\{\mathrm{d}_{\widehat{\mathcal{C}} F}(x, y)\right\}\right\}_{s}+3 K^{\prime}\left\{\left\{\mathrm{d}_{\widehat{\mathcal{C}} \mathcal{X}}(x, y)\right\}_{s}+3 C^{\prime} K^{\prime} .\right.
\end{aligned}
$$

The lower bound: We have that $\mathrm{d}_{\mathcal{X}}(x, y) \geqslant r$ and we have the estimate $\mathbf{I}+\mathbf{I I} \leqslant$ $3 K^{\prime} \mathrm{d}_{\mathcal{X}}(x, y)+C^{\prime} r$, by the induction hypothesis and Lemma 9.3 . Hence

$$
\mathbf{I}+\mathbf{I I}+\{r\}_{s} \leqslant\left(3 K^{\prime}+C^{\prime}+1\right) \mathrm{d}_{\mathcal{X}}(x, y),
$$

and we are done.

\section{Projection of Parallelism Classes}

Let $\mathcal{X}$ be $\operatorname{CAT}(0)$ cube complex with a factor system $\mathfrak{F}$, and let $\overline{\mathfrak{F}}$ be the set of parallelism classes of elements of $\mathfrak{F}$. We have previously defined projections from $\mathcal{X}$ to factored contact graphs of elements of $\mathfrak{F}$, and noted that $\pi_{F}: \mathcal{X} \rightarrow \widehat{\mathcal{C}} F$ is independent of the choice of representative of the parallelism class $[F]$. However, care must be taken in order to define projections of parallelism classes:

Proposition 10.1 (Projections of parallelism classes are bounded or cover). Let $F^{\prime}, F^{\prime \prime} \in \mathfrak{F}$ with $F^{\prime}$ parallel to $F^{\prime \prime}$. Then one of the following holds for each $F \in \mathfrak{F}$ :

(1) $\mathrm{d}_{\widehat{\mathcal{C}} F}\left(\pi_{F}\left(F^{\prime}\right), \pi_{F}\left(F^{\prime \prime}\right)\right) \leqslant \xi+2$, where $\xi$ is a constant of the factor system;

(2) there is a cubical isometric embedding $F^{\prime} \times F \rightarrow \mathcal{X}$ such that for some $f^{\prime}, f^{\prime \prime} \in F$, the subcomplexes $F^{\prime}, F^{\prime \prime}$ are the images of $F^{\prime} \times\left\{f^{\prime}\right\}, F^{\prime} \times\left\{f^{\prime \prime}\right\}$, and for some $f \in F^{\prime}$, the subcomplex $F$ is the image of $\{f\} \times F$. In particular, $\cup_{F_{1} \in\left[F^{\prime}\right]} \pi_{F}\left(F_{1}\right)=\widehat{\mathcal{C}} F$.

Moreover, whether assertion (1) or (2) holds for $F^{\prime}, F^{\prime \prime}$ with respect to $F$ is independent of the choice of parallelism class representative of $F$. 
Proof. By Lemma 2.4, there is a convex subcomplex $B$ and a cubical isometric embedding $F^{\prime} \times B \rightarrow \mathcal{X}$ with the following properties: $B$ is the convex hull of a shortest geodesic $[0, b]$ joining $F^{\prime}$ to $F^{\prime \prime}$ and $F^{\prime}, F^{\prime \prime}$ are respectively the images of $F^{\prime} \times\{0\}$ and $F^{\prime} \times\{b\}$, and in fact $[0, b]$ joins $\mathfrak{g}_{F^{\prime}}\left(F^{\prime \prime}\right)$ to $\mathfrak{g}_{F^{\prime \prime}}\left(F^{\prime}\right)$ (the fact that $[0, b]$ is shortest and joins $\mathfrak{g}_{F^{\prime}}\left(F^{\prime \prime}\right)$ to $\mathfrak{g}_{F^{\prime \prime}}\left(F^{\prime}\right)$ follows from the fact that it only crosses the hyperplanes that separate $F^{\prime}$ from $F^{\prime \prime}$, which is part of Lemma 2.4. Since $B$ is, at minimum, contained in a combinatorial hyperplane (indeed, cubical isometric embeddings take hyperplanes to hyperplanes), it is contained in some minimal $Y \in \mathfrak{F}$. If $b \leqslant \xi$, then the first assertion holds. Hence suppose $b \geqslant \xi$. Then either $\pi_{F}(Y)$ has diameter at most $\xi+2$, in which case the first assertion again holds, or by Lemma 8.19, $F$ is parallel to a subcomplex $F_{1}$ of $Y$ containing $B$. By minimality of $Y$, we have $F_{1}=Y$. Similarly, for all $f \in F^{\prime}$, we have a parallel copy $\{f\} \times F_{1}$ of $F$ containing $\{f\} \times B$. Item (2) follows.

Definition 10.2 (Orthogonal). Let $\left[F_{1}\right],\left[F_{2}\right] \in \overline{\mathfrak{F}}$. If there are $F_{1}^{\prime}, F_{1}^{\prime \prime} \in\left[F_{1}\right]$ such that Proposition 10.1. (2) holds for $F_{1}^{\prime}, F_{1}^{\prime \prime}$ projected to $F_{2}$, then $\left[F_{1}\right]$ and $\left[F_{2}\right]$ (and $F_{1}$ and $F_{2}$ ) are said to be orthogonal.

Remark 10.3. Lemma 8.19 implies that either $F$ is parallel to a subcomplex of $F^{\prime}$ or $\pi_{F}\left(F^{\prime}\right)$ has uniformly bounded diameter. In the latter case, Proposition 10.1 implies that either $\cup_{F_{1} \in\left[F^{\prime}\right]} \pi_{F}\left(F_{1}\right)$ is uniformly bounded, or $[F]$ and $\left[F_{1}\right]$ are orthogonal. In fact, Lemma 8.19 says that if $F$ is not parallel to a subcomplex of $F^{\prime}$, then $\pi_{F}\left(F^{\prime \prime}\right)$ has diameter bounded by $\xi+2$ for each $F^{\prime \prime}$ parallel to $F^{\prime}$. On the other hand, whenever $\pi_{F}\left(F^{\prime \prime}\right)$ has diameter bounded by $\xi+2$ for each $F^{\prime \prime}$ parallel to $F^{\prime}$, Proposition 10.1 implies that either $\cup_{F_{1} \in\left[F^{\prime}\right]} \pi_{F}\left(F_{1}\right)$ has diameter bounded by $3 \xi+6$ or $[F]$ and $\left[F^{\prime}\right]$ are orthogonal.

Motivated by the remark, we give the following two definitions. Definition 10.5 provides projections between parallelism classes; as explained in Remark 10.3, Proposition 10.1 gives conditions ensuring that these projections are coarsely well-defined.

Definition 10.4 (Transverse). Parallelism classes $[F],\left[F^{\prime}\right] \in \overline{\mathfrak{F}}$ are transverse if they are not orthogonal and if $F$ is not parallel to a subcomplex of $F^{\prime}$, or vice versa, for some (hence all) $F \in[F], F^{\prime} \in\left[F^{\prime}\right]$.

Definition 10.5 (Projection of parallelism classes, projection distances). Let $[F] \in \overline{\mathfrak{F}}$. The projection to $[F]$ (really to $\widehat{\mathcal{C}} F$, which depends only on the parallelism class) is the map $\overline{\mathfrak{F}}-\{[F]\} \rightarrow 2^{\widehat{\mathcal{C}} F}$ given by $\pi_{[F]}\left(\left[F^{\prime}\right]\right)=\cup_{F_{1} \in\left[F^{\prime}\right]} \pi_{F}\left(F_{1}\right)$.

For each $[Y] \in \overline{\mathfrak{F}}$, define a function $\mathrm{d}_{Y}^{\pi}:(\overline{\mathfrak{F}}-\{[Y]\})^{2} \rightarrow[0, \infty]$ by

$$
\mathrm{d}_{Y}^{\pi}\left([F],\left[F^{\prime}\right]\right)=\operatorname{diam}_{\hat{\mathcal{C}} Y}\left(\pi_{[Y]}([F]) \cup \pi_{[Y]}\left(\left[F^{\prime}\right]\right)\right) .
$$

We fix, until the end of the section, any subset $\overline{\mathfrak{F}}_{t r} \subset \overline{\mathfrak{F}}$ with the property that for each distinct pair $[F],\left[F^{\prime}\right] \in \overline{\mathfrak{F}}_{t r}$ we have that $[F]$ is transverse to $\left[F^{\prime}\right]$.

Observe that for each $F \in \overline{\mathfrak{F}}_{t r}$, the restriction of $\mathrm{d}_{F}^{\pi}$ to $\overline{\mathfrak{F}}_{t r}$ takes uniformly bounded values. From the definition, it is obvious that $\mathrm{d}_{F}^{\pi}\left(\left[F^{\prime}\right],\left[F^{\prime \prime}\right]\right)=\mathrm{d}_{F}^{\pi}\left(\left[F^{\prime \prime}\right],\left[F^{\prime}\right]\right)$ and $\mathrm{d}_{F}^{\pi}\left(\left[F^{\prime}\right],\left[F^{\prime \prime}\right]\right)+$ $\mathrm{d}_{F}^{\pi}\left(\left[F^{\prime \prime}\right],\left[F^{\prime \prime \prime}\right]\right) \geqslant \mathrm{d}_{F}^{\pi}\left(\left[F^{\prime}\right],\left[F^{\prime \prime \prime}\right]\right)$ for all $[F],\left[F^{\prime}\right],\left[F^{\prime \prime}\right],\left[F^{\prime \prime \prime}\right] \in \overline{\mathfrak{F}}_{t r}$. Moreover, the fact that the right-hand side of the distance formula (Theorem 9.1) is finite shows that, for a suitable $\eta$, we have $\left|\left\{F: \mathrm{d}_{F}^{\pi}\left(\left[F^{\prime}\right],\left[F^{\prime \prime}\right]\right) \geqslant \eta\right\}\right|<\infty$ for all $\left[F^{\prime}\right],\left[F^{\prime \prime}\right] \in \overline{\mathfrak{F}}_{t r}$.

Proposition 10.6. For all $[F] \neq\left[F^{\prime}\right]$ in $\overline{\mathfrak{F}}_{t r}$, we have $\operatorname{diam}_{\widehat{\mathcal{C}} F}\left(\pi_{F}\left(F^{\prime}\right)\right) \leqslant \xi+2$.

Proof. This is just Lemma 8.19 combined with the fact that $F$ cannot be parallel to a subcomplex of $F^{\prime}$ by our assumptions on $\overline{\mathfrak{F}}_{t r}$. 
The next result is the analogue for cubical groups of the inequality that Behrstock established in Beh06] for the mapping class group. Versions of this inequality in other contexts have appeared in $\mathrm{BBFb}, \mathrm{BF}, \mathrm{SS}$, Tay

Proposition 10.7 (Behrstock inequality). For all $[F],\left[F^{\prime}\right],\left[F^{\prime \prime}\right] \in \overline{\mathfrak{F}}_{t r}$,

$$
\min \left\{\mathrm{d}_{F}^{\pi}\left(\left[F^{\prime}\right],\left[F^{\prime \prime}\right]\right), \mathrm{d}_{F^{\prime}}^{\pi}\left([F],\left[F^{\prime \prime}\right]\right)\right\} \leqslant 3 \xi+6 .
$$

Proof. Let $F, F^{\prime}, F^{\prime \prime}$ satisfy $\mathrm{d}_{F}^{\pi}\left(\left[F^{\prime}\right],\left[F^{\prime \prime}\right]\right)>3 \xi+6$. Let $x \in F^{\prime}$. We claim that there exists $Y$ parallel to $F$ and $y \in Y$ so that $\mathfrak{g}_{F^{\prime \prime}}(x)=\mathfrak{g}_{F^{\prime \prime}}(y)$, which will conclude the proof.

Let $\gamma$ be a geodesic from $x$ to $\mathfrak{g}_{F^{\prime \prime}}(x)$. By Lemma 10.8, there exists $Y$ parallel to $F$ such that $\gamma$ intersects $Y$, say at $y$. Since $y$ is on $\gamma$, we have $\mathfrak{g}_{F^{\prime \prime}}(x)=\mathfrak{g}_{F^{\prime \prime}}(y)$, as required.

Lemma 10.8. Let $F \in \mathfrak{F}$ and let $x, y \in \mathcal{X}^{(0)}$ satisfy $\mathrm{d}_{\widehat{\mathcal{C}} F}\left(\pi_{F}(x), \pi_{F}(y)\right)>2 \xi+4$. Then any geodesic from $x$ to $y$ enters a parallel copy of $F$.

Proof. Let $\gamma$ be a geodesic from $x$ to $y$. By Lemma 8.17 - which implies $\mathrm{d}_{\widehat{\mathcal{C}} F}\left(\pi_{F}(a), \pi_{F}(b)\right)=$ $\mathrm{d}_{\widehat{\mathcal{C}} F^{\prime}}\left(\pi_{F^{\prime}}(a), \pi_{F^{\prime}}(b)\right)$ for all $a, b \in \mathcal{X}$ whenever $F, F^{\prime}$ are parallel - we can assume that $F$ was chosen within its parallelism class so that $\mathrm{d}_{\mathcal{X}}(F, \gamma)$ is minimal.

Observe that no hyperplane separates $\gamma$ from $F$. Indeed, suppose that $H$ is such a hyperplane. Then $\mathfrak{g}_{F}(x), \mathfrak{g}_{F}(y) \subset \mathfrak{g}_{F}(H)$. By our assumption that $\mathrm{d}_{\widehat{\mathcal{C}} F}\left(\pi_{F}(x), \pi_{F}(y)\right)>2 \xi+4$, we have $\operatorname{diam}_{\hat{\mathcal{C}} F}\left(\mathfrak{g}_{F}(H)\right)>2 \xi+4>\xi+2$, whence, by Lemma 8.19, $F$ is parallel to a subcomplex $F^{\prime}$ of the combinatorial hyperplane $H^{-}$on $\mathcal{N}(H)$ which is separated from $F$ by $H$. For any $z \in \gamma$, any hyperplane separating $z$ from $F^{\prime}$ separates $z$ from $F$, whence $\mathrm{d}_{\mathcal{X}}\left(\gamma, F^{\prime}\right)<\mathrm{d}_{\mathcal{X}}(\gamma, F)$, contradicting our choice of $F$.

Let $\mathfrak{L}$ be the set of hyperplanes separating $x$ from $\{y\} \cup F$ and let $\mathfrak{R}$ be the set of hyperplanes separating $y$ from $\{x\} \cup F$. If $\mathfrak{L}=\varnothing$, then either $x \in F$, since no hyperplane separates $x$ and $y$ from $F$ (for otherwise, by convexity of halfspaces, that hyperplane would separate $\gamma$ from $F$ ). Similarly, $\mathfrak{R} \neq \varnothing$.

Let $L \in \mathfrak{L}$ be closest to $y$ and let $R \in \mathfrak{R}$ be closest to $x$. Suppose that $L \cap R=\varnothing$. Then there exists a 0 -cube $z \in \gamma$ so that no hyperplane in $\mathfrak{L} \cup \mathfrak{R}$ separates $z$ from $F$. Hence any hyperplane separating $z$ from $F$ separates $\{x, y\}$, and thus $\gamma$, from $F$, which is impossible.

Thus $L$ and $R$ cross. Since $\mathfrak{g}_{F}(x), \mathfrak{g}_{F}(y) \in \mathfrak{g}_{F}(L) \cup \mathfrak{g}_{F}(R)$, arguing as above yields, say, $\operatorname{diam}_{\widehat{\mathcal{C}} F}\left(\mathfrak{g}_{F}(L)\right)>\xi+2$, so $F$ is parallel into $L$, violating our choice of $F$ as above.

Observe that Propositions 10.6 and 10.7 together with the discussion preceding them, imply the following, which we record for later convenience:

Corollary 10.9 (BBF Axioms). Let $\mathfrak{F}$ be a factor system and let $\overline{\mathfrak{F}}$ be the set of parallelism classes in $\mathfrak{F}$. Let $\overline{\mathfrak{F}}_{t r}$ be a subset of $\overline{\mathfrak{F}}$ such that $[F],\left[F^{\prime}\right]$ are transverse for all distinct $[F],\left[F^{\prime}\right] \in \overline{\mathfrak{F}}_{t r}$. Then $\left\{\mathrm{d}_{Y}^{\pi}:[Y] \in \overline{\mathfrak{F}}_{t r}\right\}$ satisfies Axioms $(0)-(4)$ of $[\mathrm{BBFb}$, Section 2.1].

\section{EFFICIENT EMBEDdings into PRODUCTS OF TREES}

We now let $\mathcal{X}$ be a $\operatorname{CAT}(0)$ cube complex with a proper cocompact action by a group $G$, and we let $\mathfrak{F}$ be a $G$-invariant factor system (recall that the existence of any factor system ensures the existence of a $G$-invariant one). In this section we produce a very particular $G$-equivariant quasi-isometric embedding of $\mathcal{X}$ into the product of finitely many quasi-trees. We begin with the following fact which is well-known in the study of cubical groups:

Proposition 11.1 (Embeddings in products of trees). Suppose that there exists $G^{\prime} \leqslant f_{\text {.i. }} G$ such that no hyperplane in $\mathcal{X}$ crosses its $G^{\prime}$-translates. Then there exists a finite collection $\left\{T_{1}, \ldots, T_{k}\right\}$ of simplicial trees, a $G$-action on $\prod_{i=1}^{k} T_{i}$, and a $G$-equivariant isometric embedding $\mathcal{X} \rightarrow \prod_{i=1}^{k} T_{i}$. 
Conversely, suppose that $G$ acts properly and cocompactly on a CAT(0) cube complex $\mathcal{X}$, and that there is a $G$-equivariant cubical isometric embedding $\mathcal{X} \rightarrow \prod_{i=1}^{k} T_{i}$ with each $T_{i}$ a simplicial tree. Then there exists a finite-index subgroup $G^{\prime} \leqslant G$ such that for all hyperplanes $H$ of $\mathcal{X}$ and all $g \in G^{\prime}$, the hyperplanes $g H$ and $H$ do not cross.

Proof. By passing to the normal core, we can assume $G^{\prime}$ is normal. Let $H_{1}, \ldots, H_{k}$ be a complete list of representatives of $G^{\prime}$-orbits of hyperplanes. For each $i$, the cube complex dual to the wallspace $\left(\mathcal{X}^{(0)}, G^{\prime} \cdot H_{i}\right)$ is a simiplicial tree $T_{i}$ on which $G^{\prime}$ acts by isometries, and $G$ acts on $\prod_{i=1}^{k} T_{k}$, permuting the factors, with $G^{\prime}$ stabilizing each factor (if $h \in G^{\prime}$ and $g \in G$ and $H_{j}=g H_{i}$, then $h H_{j}=h g H_{i}=g\left(g^{-1} h g\right) H_{i}=g H_{i}$ since $G^{\prime}$ is normal). The existence of the embedding follows from [CH13, Corollary 1]; equivariance is easily checked.

Conversely, let $\mathcal{X} \rightarrow \prod_{i=1}^{k} T_{i}$ be a $G$-equivariant embedding, and let $G^{\prime}$ be the kernel of the action of $G$ on the factors of $\prod_{i=1}^{k} \mathcal{X}$. Since $\mathcal{X} \rightarrow \prod_{i=1}^{k} T_{i}$ sends hyperplanes to hyperplanes, and sends crossing hyperplanes to crossing hyperplanes, the coloring of the hyperplanes of $\prod_{i=1}^{n} T_{i}$ by the tree factor that they cross pulls back to a $G^{\prime}$-invariant coloring of the hyperplanes of $\mathcal{X}$ by $k$ colors, with the property that hyperplanes of like color do not cross. By definition, two hyperplanes in the same $G^{\prime}$ orbit have the same color.

An element of $\mathfrak{F}-\{\mathcal{X}\}$ is maximal if it is not properly contained in an element of $\mathfrak{F}-\{\mathcal{X}\}$.

Definition 11.2 (Hereditarily flip-free). Let $G$ act properly and cocompactly on the cube complex $\mathcal{X}$. The action is flip-free (with respect to $\mathfrak{F}$ ) if for each cubical isometric embedding $A \times B \rightarrow \mathcal{X}$ such that $A, B \in \mathfrak{F}-\{\mathcal{X}\}$ and each of $A, B$ is parallel to a maximal element of $\mathfrak{F}-\{\mathcal{X}\}$, no $g \in G$ has $g A$ parallel to $B$. The action is hereditarily flip-free with respect to $\mathfrak{F}$ if for each $H \in \mathfrak{F}$, the action of $\operatorname{Stab}_{G}(H)$ on $H$ is flip-free with respect to $\mathfrak{F}_{H}$.

Lemma 11.3 (Coloring the factor system). Let $G$ act properly and cocompactly on $\mathcal{X}$, let $\mathfrak{F}$ be a $G$-invariant factor system, and suppose that there exists $G^{\prime} \leqslant f_{\text {.i. }}$. $G$ such that the action of $G^{\prime}$ on $\mathcal{X}$ is hereditarily flip-free with respect to $\mathfrak{F}$. Then there exists $k \in \mathbb{N}$ and a coloring $\chi: \mathfrak{F} \rightarrow\{1, \ldots, k\}$ such that:

(1) if $F, F^{\prime} \in \mathfrak{F}$ are parallel, then $\chi(F)=\chi\left(F^{\prime}\right)$;

(2) $\chi(g F)=\chi(F)$ for all $F$ and all $g \in G^{\prime}$;

(3) if $F$ is parallel to a proper subcomplex of $F^{\prime}$, then $\chi(F) \neq \chi\left(F^{\prime}\right)$;

(4) if $[F]$ and $\left[F^{\prime}\right]$ are orthogonal, then $\chi(F) \neq \chi\left(F^{\prime}\right)$.

Hence there is a $G^{\prime}$-invariant coloring $\chi: \overline{\mathfrak{F}} \rightarrow\{1, \ldots, k\}$ such that for $1 \leqslant i \leqslant k$, the set $\overline{\mathfrak{F}}_{i}=\chi^{-1}(i)$ is a $G^{\prime}$-invariant collection satisfying the hypotheses of Corollary 10.9.

Proof. For $n \geqslant 1$ and $\left\{q_{i}\right\}_{i=1}^{n}$ determined below, let $\left\{c_{1}^{0}, \ldots, c_{q_{n}}^{0}\right\},\left\{c_{1}^{1}, \ldots, c_{q_{1}}^{1}\right\} \ldots,\left\{c_{1}^{n}, \ldots, c_{q_{n}}^{n}\right\}$ be disjoint sets of colors, and let $\varepsilon$ be an extra color.

Define the relation $\sim$ on $\mathfrak{F}$ to be the transitive closure of the union of the following two relations: $F \sim F^{\prime}$ if $F, F^{\prime}$ are parallel; $F \sim F^{\prime}$ if there exists $g \in G^{\prime}$ such that $F=g F$. Since the $G$-action preserves parallelism, if $F \sim F^{\prime}$, then there exists $g \in G^{\prime}$ with $F, g F^{\prime}$ parallel.

We say that $F \in \mathfrak{F}-\{\mathcal{X}\}$ is maximal if it is not parallel to a proper subcomplex of any other $F^{\prime} \in \mathfrak{F}-\{\mathcal{X}\}$. Notice that maximal elements exist. In fact, if we had a chain $F_{1}, \ldots, F_{N}$, with $N$ larger than the local finiteness constant $\Delta$ of the factor system, so that $F_{i}$ is parallel to a proper subcomplex of $F_{i+1}$, then there would be some 0 -cube in $\bigcap_{i} \mathfrak{g}_{F_{N}}\left(F_{i}\right)$, violating local finiteness. Let $F_{1}, \ldots, F_{n} \in \mathfrak{F}$ be maximal elements of $\mathfrak{F}-\{\mathcal{X}\}$, chosen so that any other maximal $F \in \mathfrak{F}-\{\mathcal{X}\}$ satisfies $F \sim F_{i}$ for some unique $i$; there are finitely many $\sim$-classes since our hypotheses ensure that there are finitely many $G$-orbits in $\mathfrak{F}$. Let $\mathfrak{F}_{0} \subseteq \mathfrak{F}$ consist of those $F$ such that $F \sim F_{i}$ for some $i$, so that each maximal factor subcomplex is in $\mathfrak{F}_{0}$. Define $\chi_{0}: \mathfrak{F}_{0} \rightarrow\left\{c_{1}^{0}, \ldots, c_{n}^{0}\right\}$ by $\chi_{0}(F)=c_{i}^{0}$, where $i$ is the unique index with $F \sim F_{i}$. The coloring $\chi_{0}$ of $\mathfrak{F}_{0}$ satisfies items (1), (2), (3) by definition (in particular, maximality ensures 
that item (3) holds). Item (4) follows from the assumption that the action of $G^{\prime}$ is flip-free. Indeed, if $[F]$ and $\left[F^{\prime}\right]$ are orthogonal then, by definition, we can choose $F, F^{\prime}$ within their parallelism classes so that $F \times F^{\prime} \hookrightarrow \mathcal{X}$ isometrically. But if $\chi_{0}(F)=\chi_{0}\left(F^{\prime}\right)$, then $F$ is parallel to $g F^{\prime}$ for some $g \in G^{\prime}$, contradicting flip-freeness since each of $F, F^{\prime}$ is parallel to a maximal element, namely a translate of some $F_{i}$.

Note that since $\mathfrak{F}_{0}$ is a locally finite collection and $G^{\prime}$ acts on $\mathcal{X}$ cocompactly, $G_{F}^{\prime}:=$ $\operatorname{Stab}_{G^{\prime}}(F)$ acts cocompactly on $F$ for each $F \in \mathfrak{F}$. For each $F \in \mathfrak{F}$, let $\mathfrak{F}_{F}$ be the induced factor system, so that $\cup_{F} \mathfrak{F}_{F}=\mathfrak{F}$. By hereditary flip-freeness of the overall action of $G^{\prime}$ on $\mathcal{X}$, the action of $G_{F}^{\prime}$ on each $F$ is flip-free with respect to $\mathfrak{F}_{F}$. Hence, by induction, for $1 \leqslant i \leqslant n$, we have a coloring $\chi_{F_{i}}^{\prime}: \mathfrak{F}_{F_{i}} \rightarrow\left\{c_{1}^{i}, \ldots, c_{q_{i}}^{i}\right\}$ satisfying all four conclusions of the proposition with respect to the $G_{F_{i}}^{\prime}$-action.

We define $\chi_{i}: \cup F \sim F_{i} \mathfrak{F}_{F} \rightarrow\left\{c_{1}^{i}, \ldots, c_{q_{i}}^{i}\right\}$ as follows. If $F$ is parallel to $F_{i}$, then each $F^{\prime} \in \mathfrak{F}_{F}$ is parallel to some $F_{i}^{\prime} \in \mathfrak{F}_{F_{i}}$, and if $F^{\prime}$ is also parallel to $F_{i}^{\prime \prime} \in \mathfrak{F}_{F_{i}}$, then $\chi_{F_{i}}^{\prime}\left(F_{i}^{\prime}\right)=\chi_{F_{i}}^{\prime}\left(F_{i}^{\prime \prime}\right)$. Hence let $\chi_{i}\left(F^{\prime}\right)=\chi_{F_{i}}\left(F_{i}^{\prime}\right)$. We now extend $G^{\prime}$-equivariantly to obtain $\chi_{i}$. More precisely, for each $F \sim F_{i}$, choose $g \in G^{\prime}$ so that $g F$ is parallel to $F_{i}$, and define $\chi_{i}$ on $\mathfrak{F}_{F}$ so that $\chi_{i}\left(F^{\prime}\right)=\chi_{i}\left(g F^{\prime}\right)$ for all $F^{\prime} \in \mathfrak{F}_{F}$. This is independent of the choice of $g F$ because of how $\chi_{i}$ was defined on the subcomplexes parallel to $F_{i}$. Moreover, since $\chi_{F_{i}}^{\prime}$ is $G_{F_{i}}^{\prime}$-invariant, and parallel complexes have identical stabilizers, this is independent of the choice of $g$.

Let $F \sim F_{i}$ and let $F^{\prime}, F^{\prime \prime} \in \mathfrak{F}_{F}$. Suppose that $\chi_{i}\left(F^{\prime}\right)=\chi_{i}\left(F^{\prime \prime}\right)$. Choose $g \in G^{\prime}$ so that $g F$ is parallel to $F_{i}$. Then $\chi_{i}\left(g F^{\prime}\right)=\chi_{i}\left(g F^{\prime \prime}\right)=\chi_{i}\left(F_{i}^{\prime}\right)=\chi_{i}\left(F_{i}^{\prime \prime}\right)$, where $F_{i}^{\prime}, F_{i}^{\prime \prime} \in \mathfrak{F}_{F_{i}}$ are parallel to $g F^{\prime}, g F^{\prime \prime}$ respectively. Hence neither of $F_{i}^{\prime \prime}$ nor $F_{i}^{\prime}$ is parallel to a subcomplex of the other, and they are not orthogonal, by our induction hypothesis. Hence the same is true of $F^{\prime}, F^{\prime \prime}$, so that $\chi_{i}$ correctly colors $\mathfrak{F}_{F}$.

For $i \geqslant 0$, we extend $\chi_{i}$ to the rest of $\mathfrak{F}$ by letting $\chi_{i}(H)=\varepsilon$ when $H \notin \cup_{F \sim F_{i}} \mathfrak{F}_{F}$ (or $H \notin \mathfrak{F}_{0}$ when $\left.i=0\right)$. We finally define the coloring $\chi$ on $\mathfrak{F}$ by

$$
\chi(F)=\left(\chi_{0}(F), \chi_{1}(F), \ldots, \chi_{n}(F)\right) .
$$

(The total number of colors is $(n+1) \prod_{i=1}^{n}\left(q_{i}+1\right)$.)

Let $F, F^{\prime} \in \mathfrak{F}$. If the set of $i$ for which $F$ is contained in $\mathfrak{F}_{H}$ for some $H \sim F_{i}$ differs from the corresponding set for $F^{\prime}$, then $\chi(F) \neq \chi\left(F^{\prime}\right)$ since there is at least one coordinate in $\chi(F)$ equal to $\varepsilon$ for which the corresponding coordinate in $\chi\left(F^{\prime}\right)$ is not $\varepsilon$, or vice versa. It follows from the definition of the $\chi_{i}$ that $F$ cannot be parallel to a proper subcomplex of $F^{\prime}$, and they cannot be orthogonal, if $\chi(F)=\chi\left(F^{\prime}\right)$. Hence $\chi$ is the desired coloring.

We can now prove Theorem F:

Proposition 11.4 (Embedding in a product of quasi-trees). Let $G, \mathcal{X}$, and $\overline{\mathfrak{F}}$ be as in Lemma 11.3. Then there is a partition $\overline{\mathfrak{F}} \cup\{[\mathcal{X}]\}=\sqcup_{i=1}^{k} \overline{\mathfrak{F}}_{i}$ such that each $\mathfrak{C}\left(\overline{\mathfrak{F}}_{i}\right)$ is a quasi-tree, $G$ acts by isometries on $\prod_{i=1}^{k} \mathfrak{C}\left(\overline{\mathfrak{F}}_{i}\right)$, and there is a $G$-equivariant quasi-isometric embedding $\mathcal{X} \rightarrow \prod_{i=1}^{k} \mathfrak{C}\left(\overline{\mathfrak{F}}_{i}\right)$.

Proof. Lemma 11.3 allows us to finitely and equivariantly color the elements of $\mathfrak{F}$ so that elements of the same color are transverse. By Corollary 10.9 and [BBFb, Theorem A], each color gives rise to a quasi-tree and a map from $\mathcal{X}$ to each quasi-tree. Comparing Theorem 9.1 to the distance estimate [BBFb, Theorem 4.13] shows, as in [BBFb, Section 5.3], that these maps give the desired quasi-isometric embedding in the product of the quasi-trees associated to the various colors.

Remark 11.5. Proposition 11.6 shows that Proposition 11.1 does not imply Proposition 11.4 Any stipulation that no hyperplane crosses its $G^{\prime}$-translates is satisfied when, for example, hyperplane-stabilizers are separable (this uses Scott's criterion for separability [Sco78]). Such 
separability occurs when the action of $G$ on $\mathcal{X}$ is virtually cospecial (e.g., when $G$ is wordhyperbolic Ago13); this is stronger than assuming the action of $G$ on $\mathcal{X}$ is virtually flip-free.

Proposition 11.6. There exists a group $G$ acting properly and cocompactly on a CAT(0) cube complex $\mathcal{X}$ in such a way that $\mathcal{X}$ admits a $G$-equivariant quasi-isometric embedding into the product of finitely many quasi-trees, but $\mathcal{X}$ does not admit a $G^{\prime}$-equivariant isometric embedding in a finite product of trees for any finite-index $G^{\prime} \leqslant G$.

Proof. We will actually find $G$ and $\mathcal{X}$ such that the following three properties hold:

(1) $\mathcal{X}$ admits a factor system $\mathfrak{F}$.

(2) For every finite-index subgroup $G^{\prime} \leqslant G$, there exists a hyperplane $H$ of $\mathcal{X}$ and some $g \in G^{\prime}$ such that the hyperplanes $H, g H$ cross.

(3) The action of $G^{\prime \prime}$ on $\mathcal{X}$ is hereditarily flip-free for some $G^{\prime \prime} \leqslant f_{\text {.i. }} G$.

Let $Z$ be the complex from Example 8.10, whose notation we will use throughout this proof, let $G=\pi_{1} Z$, and let $\mathcal{X}=\widetilde{Z}$. Property (1) is Proposition 8.11 and Property (2) is Proposition 8.12. Thus, by Proposition 11.1, $\mathcal{X}$ has no $G$-equivariant isometric embedding in a finite product of trees. The existence of the desired quasi-isometric embedding will follow from Proposition 11.4 once we have established property (3).

To that end, it will be convenient to compute an explicit factor system for $\mathcal{X}$. We take $\xi \geqslant r+1$ and let $\mathfrak{F}$ be the smallest subset containing $\mathcal{X}$, all combinatorial hyperplanes of $\mathcal{X}$, and $\mathfrak{g}_{F}\left(F^{\prime}\right)$ whenever $F, F^{\prime} \in \mathfrak{F}$ and $\operatorname{diam}\left(\mathfrak{g}_{F}\left(F^{\prime}\right)\right) \geqslant \xi$.

We first describe the combinatorial hyperplanes in $\mathcal{X}$. We can and shall assume that the paths $\alpha, \beta:[0, r] \rightarrow S, T$ from Example 8.10 are surjective. This is for convenience only; it reduces the number of isomorphism types of hyperplane that we need to consider. Let $\mathcal{X}_{0}$ be the universal cover of $Z_{0}$, which decomposes as a tree of spaces whose vertex-spaces are copies of $\widetilde{S} \times \widetilde{T}$ and whose edge-spaces are lines. Then $\mathcal{X}$ is formed from $\mathcal{X}_{0}$ by attaching all lifts of the carrier of the self-crossing hyperplane $W$ (which is a segment of length $r+2$ ) along a segment of length $r$.

$\mathcal{X}$ thus has compact hyperplanes, all of which are lifts of $W$. Each corresponds to a cardinality-2 parallelism class of combinatorial hyperplanes. The projection of such a combinatorial hyperplane $W^{+}$onto some other hyperplane is either an isomorphism or has image of diameter at most $r$, whence this projection is excluded from $\mathfrak{F}$ by our choice of $\xi$. The projection of any other hyperplane onto $W^{+}$also has diameter at most $r$ and is similarly excluded. Every product subcomplex of the form $W^{+} \times E$ with $W^{+}$a compact combinatorial hyperplane has the property that $E$ is contained in a 1 -cube and thus is not a combinatorial hyperplane. Hence the compact hyperplanes do not affect whether the action of $G$ is flip-free.

The remaining combinatorial hyperplanes are of two types: there are two-ended combinatorial hyperplanes, which are parallel to lifts of $\widetilde{C}$, and bushy hyperplanes, that are trees of the following type. Each is formed by beginning with a copy of $\widetilde{S}$, attaching a path of length 2 to each vertex, attaching a copy of $\widetilde{T}$ to the end of each of these paths, etc. Then, at the midpoint of each of the length-2 paths (i.e., the points at which the hyperplane intersects a lift of $\widetilde{C}$ ), attach a single 1-cube (dual to a compact hyperplane). Observe that any subcomplex parallel to a bushy hyperplane is a bushy hyperplane, but that two-ended hyperplanes are parallel to lines properly contained in bushy hyperplanes.

Let $T, T^{\prime}$ be subcomplexes parallel to two-ended hyperplanes and let $B, B^{\prime}$ be bushy hyperplanes. If $B, B^{\prime}$ intersect some lift of $\widetilde{S} \times \widetilde{T}$ but do not intersect, then $B$ and $B^{\prime}$ are parallel, so that $\mathfrak{g}_{B}\left(B^{\prime}\right)=B$. If $B, B^{\prime}$ are distinct and intersect, then $\mathfrak{g}_{B}\left(B^{\prime}\right)$ is a single point. If $B, B^{\prime}$ do not intersect a common vertex-space, then $\mathfrak{g}_{B}\left(B^{\prime}\right)=\mathfrak{g}_{B}(T)$ for some two-ended hyperplane separating $B$ from $B^{\prime}$. But $\mathfrak{g}_{B}(T)$ is either a single point or is a parallel copy of 
$T$ for any bushy $B$ and two-ended $T$. Hence $\mathfrak{F}$ consists of $\mathcal{X}$, combinatorial hyperplanes and their parallel copies. Maximal elements of $\mathfrak{F}-\{\mathcal{X}\}$ are bushy hyperplanes or lifts of $\widetilde{C}$.

Let $G^{\prime \prime}$ be the unique index-2 subgroup of $G$ (this is the kernel of the map $G \rightarrow \mathbb{Z}_{2}$ sending the element represented by $C$ to 1$)$. Then the action of $G^{\prime \prime}$ on $\mathcal{X}$ is flip-free. Indeed, $\mathcal{X}$ does not contain the product of two bushy hyperplanes, so we need only consider the case of a product $\widetilde{\alpha} \times \widetilde{\beta}$ in some $\widetilde{S} \times \widetilde{T}$. But any $G^{\prime \prime}$-translate of $\widetilde{\beta}$ is either in the same product piece - and thus parallel to $\beta$ - or at even distance from $\widetilde{\beta}$ in the Bass-Serre tree, and hence is again a component of the preimage of $\beta$, and hence not parallel to $\widetilde{\alpha}$. To verify hereditary flip-freeness, it suffices to note that each element of $\mathfrak{F}-\{\mathcal{X}\}$ is a tree, and thus contains no nontrivial products.

\section{Consistency and Realization}

In this section, we prove an analogue in the cubical context of the Consistency Theorem in the mapping class group; see [BKMM12, Theorem 4.3]. (For an analogue of this theorem in Teichmüller space with the Teichmüller metric, see [EMR, Section 3].) For the purposes of this section, $\mathcal{X}$ is an arbitrary $\mathrm{CAT}(0)$ cube complex with a factor system $\mathfrak{F}$. Let $\xi$ be the constant from Definition 8.1. For each $F \in \mathfrak{F}$, let $\mathfrak{B} F=\left\{S \subseteq \mathcal{C} F^{(0)}: \operatorname{diam}_{\widehat{\mathcal{C}} F}(S) \leqslant 1\right\}$.

If $U, V \in \mathfrak{F}$, and $V$ is parallel to a proper subcomplex of $U$, then there is a map $\rho_{V}^{U}: \mathfrak{B} U \rightarrow$ $2^{\hat{\mathcal{C}} V^{(0)}}$ defined as follows: let $b \in \mathfrak{B} U$ be a clique in $\mathcal{C} U$ and let $\rho_{V}^{U}(b)=\cup_{W} \pi_{V}(W)$, where $W$ varies over all combinatorial hyperplanes parallel to hyperplanes of $U$ in the clique $b$.

Let $\vec{b} \in \prod_{[F] \in \overline{\mathfrak{F}}} \mathfrak{B} F$ be a tuple, whose $[F]$-coordinate we denote by $b_{F}$. The tuple $\vec{b}$ is realized if there exists $x \in \mathcal{X}$ such that $\pi_{F}(x)=b_{F}$ for all $[F] \in \overline{\mathfrak{F}}$; note that by Lemma 8.17 $b_{F}$ is independent of the choice of representative $F \in[F]$. In this section we first give a set of consistency conditions on coordinate projections which hold for any element of $x \in \mathcal{X}$ (for the analogue in the mapping class group see [Beh06]). We then show that this set of necessary consistency conditions on a vector of coordinates is, essentially, also sufficent for that vector to be realized by an element of $\mathcal{X}$.

Remark 12.1. In this section, we use the sets $\mathfrak{B} F$, simply because the projection of a point to the contact graph is always a clique. In the more general context introduced in Section 13 — specifically in Definition 13.1 — we abstract the conditions on tuples in $\prod_{[F] \in \overline{\mathfrak{F}}} \mathfrak{B} F$ provided by the next several results, in terms of tuples in $\prod_{S \in \mathfrak{S}} 2^{S}$, where $\mathfrak{S}$ is some set of uniformly hyperbolic spaces, and the tuple restricted to each coordinate is a uniformly bounded (though not necessarily diameter-1) set. In the cubical setting, instead of doing this using $S=\widehat{\mathcal{C}} F$, we instead use $\mathfrak{B} F$ because it yields more refined statements.

Recall that the parallelism classes $[F],\left[F^{\prime}\right] \in \overline{\mathfrak{F}}$ are transverse if they are not orthogonal and if $F$ is not parallel to a subcomplex of $F^{\prime}$, or vice versa, for some (hence all) $F \in$ $[F], F^{\prime} \in\left[F^{\prime}\right]$. We begin with the consistency conditions, which take the form of the following inequalities:

Proposition 12.2 (Realized tuples are consistent). Let $\vec{b} \in \prod_{[F] \in \overline{\mathfrak{F}}} \mathfrak{B} F$ be realized by $x \in \mathcal{X}$. There exists $\kappa_{0}=\kappa_{0}(\xi)$ such that for all $U, V \in \mathfrak{F}$, the following hold:

(1) If $U$ and $V$ are transverse, then

$$
\min \left\{\mathrm{d}_{\widehat{\mathcal{C}} U}\left(b_{U}, \pi_{U}(V)\right), \mathrm{d}_{\widehat{\mathcal{C}} V}\left(b_{V}, \pi_{V}(U)\right)\right\} \leqslant \kappa_{0} ;
$$

(2) If $V$ is parallel to a proper subcomplex of $U$, then

$$
\min \left\{\mathrm{d}_{\widehat{\mathcal{C}} U}\left(b_{U}, \pi_{U}(V)\right), \operatorname{diam}_{\widehat{\mathcal{C}} V}\left(b_{V} \cup \rho_{V}^{U}\left(b_{U}\right)\right)\right\} \leqslant \kappa_{0} .
$$


A tuple $\vec{b}$ (realized or otherwise) satisfying the conclusions of the above proposition is said to be $\kappa_{0}$-consistent. More generally, a tuple $\vec{b}$ satisfying inequalities (1) and (2) from Proposition 12.2 , with $\kappa_{0}$ replaced by some $\kappa \geqslant \kappa_{0}$, will be called $\kappa$-consistent.

Proof of Proposition 12.2. First suppose that neither $U$ nor $V$ is parallel to a subcomplex of the other. Suppose that $\mathrm{d}_{\hat{\mathcal{C}} U}\left(b_{U}, \pi_{U}(V)\right)>2 \xi+4$. Then $\mathrm{d}_{U}\left(\mathfrak{g}_{U}(x), \mathfrak{g}_{U}(V)\right)>\xi$, so that the set $\mathcal{H}_{U}$ of hyperplanes crossing $U$ and separating $x$ from $V$ has cardinality at least $\xi$. Suppose also that $\mathrm{d}_{\hat{\mathcal{C}} V}\left(b_{V}, \pi_{V}(U)\right)>2 \xi+4$, so that the set $\mathcal{H}_{V}$ of hyperplanes crossing $V$ and separating $x$ from $U$ has cardinality at least $\xi$. Let $W \in \mathcal{H}_{V}$ and let $W^{+}$be an associated combinatorial hyperplane not separated from $x$ by any hyperplane crossing $V$. Notice that $\pi_{U}\left(W^{+}\right)$contains $\pi_{U}(x)=b_{U}$ (because $W$ separates $x$ from $U$ ) and intersects $\pi_{U}(V)$. In particular $\operatorname{diam}_{\hat{\mathcal{C}} U}\left(\pi_{U}\left(W^{+}\right)\right)>\xi+2$ and hence, by Lemma 8.19, $U$ is parallel to a subcomplex $U^{\prime}$ of $W^{+}$containing $\mathfrak{g}_{W^{+}}(x)$. We have $\operatorname{diam}_{\hat{\mathcal{C}} V}\left(\pi_{V}\left(U^{\prime}\right)\right) \leqslant \xi+2$ for otherwise $V$ would be parallel into $U^{\prime}$ which is impossible since $U^{\prime}$ is parallel to $U$. Also, $\pi_{V}\left(U^{\prime}\right)$ contains $\pi_{V}(x)=b_{V}$ and hence $\mathrm{d}_{\widehat{\mathcal{C}} V}\left(\pi_{V}(U), \pi_{V}\left(U^{\prime}\right)\right)>\xi+2$, i.e., Proposition 10.1. (1) does not hold, so $U$ and $V$ are orthogonal by Proposition 10.1 .

Let $V$ be parallel to a proper subcomplex of $U$ and let $\left\{W_{i}\right\}$ be the set of combinatorial hyperplanes of $U$ containing $\mathfrak{g}_{U}(x)$. Suppose that $\mathrm{d}_{\hat{\mathcal{C}} U}\left(b_{U}, \pi_{U}(V)\right)>\xi+2$. Then for each $i$, we have that $\mathfrak{g}_{V}\left(W_{i}\right)$ is a single point (since otherwise there would be a hyperplane intersecting $\mathfrak{g}_{V}\left(W_{i}\right)$ and hence intersecting both $V$ and $\left.W_{i}\right)$, hence $\mathfrak{g}_{V}\left(W_{i}\right)=\mathfrak{g}_{V}(x)$, whence $b_{V}=\rho_{V}^{U}\left(b_{U}\right)$. In either case, $\kappa_{0}=\xi+2$ suffices.

Remark 12.3. We note that Proposition 10.7 can alternatively be proven as an immediate consequence of Proposition 12.2 and Lemma 8.19. The formulation here is very close to that in the mapping class group, see [Beh06] and [BKMM12].

Theorem 12.4 (Consistency and realization). Let $\kappa_{0} \geqslant 1$ be the constant from Proposition 12.2. For each $\kappa \geqslant \kappa_{0}$, there exists $\theta \geqslant 0$ such that, if $\vec{b} \in \prod_{[F] \in \overline{\mathfrak{F}}} \mathfrak{B} F$ is $\kappa$-consistent, then there exists $y \in \mathcal{X}$ such that for all $[F] \in \overline{\mathfrak{F}}$, we have $\mathrm{d}_{\widehat{\mathcal{C}} F}\left(b_{F}, \pi_{F}(y)\right) \leqslant \theta$.

Proof. For each $[U] \in \overline{\mathfrak{F}}$, let $E_{U}$ be the smallest convex subcomplex of $\mathcal{X}$ with the property that $y \in E_{U}$ if $\pi_{U}(y)$ intersects the $2 \kappa$-neighborhood of $b_{U}$ in $\widehat{\mathcal{C}} U$. By Lemma 8.17, we have $E_{U}=E_{U^{\prime}}$ if $[U]=\left[U^{\prime}\right]$.

Moreover, we claim that $\pi_{U}\left(E_{U}\right)$ has diameter at most $5 \kappa$. Indeed, let $\mathcal{E}$ be the set of all 0 -cubes $x$ so that $\pi_{U}(x) \cap \mathcal{N}_{2 \kappa}\left(b_{U}\right) \neq \varnothing$. Given $x, y \in \mathcal{E}$, let $H_{1}, \ldots, H_{k}$ and $V_{1}, \ldots, V_{\ell}$ be sequences of subcomplexes, representing geodesics in $\widehat{\mathcal{C}} U$, so that $x \in H_{1}, y \in V_{\ell}$, and $H_{k}, V_{1}$ correspond to points of $b_{U}$. Hence there is a combinatorial path from $x$ to $y$ and carried on $\bigcup_{i} H_{i} \cup \bigcup_{j} V_{j}$; by construction, each 0 -cube on this path lies in $\mathcal{E}$. Hence $\mathcal{E}$ is 1 -coarsely connected. Now let $z \in E_{U}-\mathcal{E}$ be a 0 -cube and let $H$ be a hyperplane whose carrier contains $z$ and which crosses a geodesic from $z$ to a point of $\mathcal{E}$. Since $E_{U}$ is the convex hull of $\mathcal{E}$, the hyperplane $H$ separates two 0 -cubes of $\mathcal{E}$ lying in $\mathcal{N}(H)$. Hence $\mathrm{d}_{\widehat{\mathcal{C}} U}\left(\pi_{U}(z), b_{U}\right) \leqslant 2 \kappa+1$ and thus $\pi_{U}\left(E_{U}\right) \leqslant 5 \kappa$.

Suppose $[U],[V] \in \overline{\mathfrak{F}}$ are transverse. By $\kappa$-consistency and Lemma 8.19 , we have that either $V \subseteq E_{U}$ or $U \subseteq E_{V}$. If $V$ is parallel to a proper subcomplex of $U$, then the same is true, by $\kappa$-consistency. Finally, if $U \times V$ isometrically embeds in $\mathcal{X}$, then some representative of $[V]$ lies in $E_{U}$ and some representative of $[U]$ lies in $E_{V}$. Hence, in all cases, at least one representative subcomplex corresponding to one of the cliques $b_{U}$ or $b_{V}$ belongs to $E_{U} \cap E_{V}$, i.e., $E_{U} \cap E_{V} \neq \varnothing$ for all $U, V$. Thus, by the Helly property, for any finite collection $\left[F_{1}\right], \ldots,\left[F_{n}\right]$ of elements of $\overline{\mathfrak{F}}$, we can find an $x \in \mathcal{X}$ so that $\mathrm{d}_{\widehat{\mathcal{C}}_{F_{i}}}\left(\pi_{F_{i}}(x), b_{F_{i}}\right) \leqslant 5 \kappa$ for each $i$.

Fix any 0 -cube $x_{0} \in \mathcal{X}$ and let $\theta_{1}$ be a (large) constant to be chosen later. Also, denote by $\overline{\mathfrak{F}}_{\text {max }}$ the set of all $[F] \in \overline{\mathfrak{F}}-\{\mathcal{X}\}$ so that 
- $\mathrm{d}_{\hat{\mathcal{C}} F}\left(\pi_{F}\left(x_{0}\right), b_{F}\right)>\theta_{1}$, and

- $F$ is not parallel to a proper subcomplex of a representative of $\left[F^{\prime}\right] \in \overline{\mathfrak{F}}-\{\mathcal{X}\}$ for which $\mathrm{d}_{\widehat{\mathcal{C}} F^{\prime}}\left(\pi_{F^{\prime}}\left(x_{0}\right), b_{F^{\prime}}\right)>\theta_{1}$.

Let $l=\mathrm{d}_{\hat{\mathcal{C}} \mathcal{X}}\left(\pi_{\mathcal{X}}\left(x_{0}\right), b_{\mathcal{X}}\right)$. We claim that $\overline{\mathfrak{F}}_{\max }$ has finitely many elements. Suppose not, and let $\left[F_{1}\right], \ldots,\left[F_{p(l+5 \kappa)+1}\right] \in \overline{\mathfrak{F}}_{\text {max }}$, for some $p$ to be determined below so that $\mathrm{d}_{\widehat{\mathcal{C}} F}\left(\pi_{F_{i}}\left(x_{0}\right), b_{F_{i}}\right)>$ $\theta_{1}$ for $1 \leqslant i \leqslant p(l+5 \kappa)+1$. Consider $x \in \mathcal{X}$ satisfying $\mathrm{d}_{\widehat{\mathcal{C}} F_{i}}\left(\pi_{F_{i}}(x), b_{F_{i}}\right) \leqslant 5 \kappa$ for each $i$ and such that $\mathrm{d}_{\hat{\mathcal{C}} \mathcal{X}}\left(\pi_{\mathcal{X}}(x), b_{\mathcal{X}}\right) \leqslant 5 \kappa$. The existence of such a point $x$ was established above.

Thus, there exists $m \leqslant l+5 \kappa$ such that $\left[T_{0}\right], \ldots,\left[T_{m}\right] \in \mathfrak{F}-\{\mathcal{X}\}$ is a sequence, with $T_{0}$ a combinatorial hyperplane parallel to a hyperplane representing a vertex of $\pi_{\mathcal{X}}\left(x_{0}\right)$ and $T_{m}$ enjoying the same property with $x$ replacing $x_{0}$, representing a geodesic in $\hat{\mathcal{C}} \mathcal{X}$ joining $\pi_{\mathcal{X}}\left(x_{0}\right)$ to $\pi_{\mathcal{X}}(x)$. Suppose that we have chosen constants $\theta_{1}$ and $\kappa$ so that $\theta_{1} \geqslant 6 \kappa-6 \geqslant 5 \kappa+4 \xi+10$. Hence, for each $i$, we have $\mathrm{d}_{\widehat{\mathcal{C}} F_{i}}\left(\pi_{F_{i}}\left(x_{0}\right), \pi_{F_{i}}(x)\right) \geqslant \theta_{1}-5 \kappa \geqslant 4 \xi+10$. Proposition 9.4 now implies that each $F_{i}$ is parallel to a subcomplex of $T_{j}$ for some $j \in\{0, \ldots, m\}$. Hence $F_{i} \in \overline{\mathfrak{F}}_{\text {max }} \cap \overline{\mathfrak{F}}_{T_{j}}$, so that it suffices to show that the latter is finite.

Note that for each $j$, the set $\overline{\mathfrak{F}}_{\text {max }} \cap \overline{\mathfrak{F}}_{T_{j}}$ is the set of maximal elements $H \in \overline{\mathfrak{F}}_{T_{j}}$ with $\mathrm{d}_{\hat{\mathcal{C}} H}\left(\pi_{H}\left(\pi_{T_{j}}\left(x_{0}\right)\right), b_{H}\right)>\theta_{1}$. This follows from the fact that $\mathfrak{g}_{H}\left(\mathfrak{g}_{T_{j}}\left(x_{0}\right)\right)=\mathfrak{g}_{H}\left(x_{0}\right)$. Either $\overline{\mathfrak{F}}_{\max } \cap \overline{\mathfrak{F}}_{T_{j}}=\left\{T_{j}\right\}$, or $\mathrm{d}_{\widehat{\mathcal{C}} T_{j}}\left(\pi_{T_{j}}\left(x_{0}\right), b_{T_{j}}\right) \leqslant \theta_{1}$ and, by induction on $\Delta$, there exists $p \geqslant 1$ with $\left|\overline{\mathfrak{F}}_{\text {max }} \cap \overline{\mathfrak{F}}_{T_{j}}\right| \leqslant p$. Hence $\left|\overline{\mathfrak{F}}_{\max }\right| \leqslant p(l+5 \kappa)$.

The finiteness of $\overline{\mathfrak{F}}_{\text {max }}$ holds for any choice of $x_{0}$, but it will be convenient for what follows to assume that $x_{0}$ has been chosen so that $\pi_{\mathcal{X}}\left(x_{0}\right)$ contains the clique $b_{\mathcal{X}}$.

As before, choose $x \in \mathcal{X}$ so that $\mathrm{d}_{\widehat{\mathcal{C}} F}\left(\pi_{F}(x), b_{F}\right) \leqslant 5 \kappa$ for each $[F] \in \overline{\mathfrak{F}}_{\text {max }}$. Let $\overline{\mathfrak{F}}_{\text {max }}^{\prime}$ be the set of all $[F] \in \overline{\mathfrak{F}}_{\text {max }}$ with the property that for each $\left[F^{\prime}\right] \in \overline{\mathfrak{F}}_{\max }$ transverse to $[F]$, the closest parallel copy of $F$ to $x$ is closer in $\mathcal{X}$ than the closest parallel copy of $F^{\prime}$ to $x$.

Claim 1. Let $\left[F_{1}\right],\left[F_{-1}\right] \in \overline{\mathfrak{F}}_{\max }$ be transverse. Then there exists a unique $i \in\{ \pm 1\}$ such that $\mathrm{d}_{\widehat{\mathcal{C}} F_{i}}\left(\pi_{F_{i}}\left(x_{0}\right), \pi_{F_{i}}\left(F_{-i}\right)\right) \leqslant \kappa$. Moreover, $\mathrm{d}_{\mathcal{X}}\left(F_{i}, x\right)<\mathrm{d}_{\mathcal{X}}\left(F_{-i}, x\right)$, assuming $F_{ \pm i}$ is the closest representative of $\left[F_{ \pm i}\right]$ to $x$.

Proof of Claim 1. Suppose first $\mathrm{d}_{\widehat{\mathcal{C}} F_{1}}\left(\pi_{F_{1}}\left(x_{0}\right), \pi_{F_{1}}\left(F_{-1}\right)\right)>\kappa$. Then, by Proposition 12.2 we have $\mathrm{d}_{\widehat{\mathcal{C}} F_{-1}}\left(\pi_{F_{-1}}\left(x_{0}\right), \pi_{F_{-1}}\left(F_{1}\right)\right) \leqslant \kappa$ and we are done. If $\mathrm{d}_{\widehat{\mathcal{C}} F_{1}}\left(\pi_{F_{1}}\left(x_{0}\right), \pi_{F_{1}}\left(F_{-1}\right)\right) \leqslant \kappa$, then $\mathrm{d}_{\widehat{\mathcal{C}} F_{1}}\left(\pi_{F_{1}}(x), \pi_{F_{1}}\left(F_{-1}\right)\right)>\kappa$. Proposition 12.2 implies that $\mathrm{d}_{\widehat{\mathcal{C}} F_{-1}}\left(\pi_{F_{-1}}(x), \pi_{F_{-1}}\left(F_{1}\right)\right) \leqslant \kappa$. But then $\mathrm{d}_{\widehat{\mathcal{C}} F_{-1}}\left(\pi_{F_{-1}}\left(x_{0}\right), \pi_{F_{-1}}\left(F_{1}\right)\right) \geqslant \theta_{1}-\kappa-\xi-2>\kappa$.

To prove the "moreover" clause, notice that $\mathrm{d}_{\widehat{\mathcal{C}} F_{i}}\left(\pi_{F_{i}}(x), \pi_{F_{i}}\left(F_{-i}\right)\right)>4 \xi+10$. Hence, by Proposition 9.4 for any point $x^{\prime}$ on any parallel copy of $F_{-i}$ there is a geodesic from $x^{\prime}$ to $x$ passing through a parallel copy of $F_{i}$.

If $\overline{\mathfrak{F}}_{\text {max }}=\varnothing$ then we can choose $y=x_{0}$. Otherwise, $\overline{\mathfrak{F}}_{\text {max }}^{\prime} \neq \varnothing$, and Claim 1 implies that the elements of $\overline{\mathfrak{F}}_{\text {max }}^{\prime}$ are pairwise-orthogonal; hence $\mathcal{X}$ contains $P=\prod_{[F] \in \overline{\mathfrak{F}}_{\max }^{\prime}} F$. By Lemma 2.4 there is a convex subcomplex $Q \subset \mathcal{X}$ such that the convex hull of the union of all subcomplexes parallel to $P$ is isomorphic to $P \times Q$. Since $|P|>1$ by our choice of $\theta_{1}$, there is at least one hyperplane crossing $P$, whence $Q$ is not unique in its parallelism class and hence lies in a combinatorial hyperplane $U$. By induction on $\Delta$, there exists $y_{U} \in U$ such that $\mathrm{d}_{\widehat{\mathcal{C}} W}\left(\pi_{W}\left(y_{U}\right), b_{W}\right) \leqslant \theta_{2}$ for some fixed $\theta_{2}$ and all $[W] \in \mathfrak{F}_{U}$. Let $y_{Q}=\mathfrak{g}_{Q}\left(y_{U}\right)$.

By induction on $\Delta$, for each $[F] \in \overline{\mathfrak{F}}_{\text {max }}^{\prime}$, we can choose $F \in[F]$ and a point $y_{F} \in F$ such that $\mathrm{d}_{\widehat{\mathcal{C}} H}\left(\pi_{H}\left(y_{F}\right), b_{H}\right) \leqslant \theta_{2}$ for all $[H] \in \mathfrak{F}_{F}$ and some $\theta_{2}$. Importantly, $\theta_{2}$ can be chosen to depend only on $\kappa, \xi$ and $\Delta-1$, i.e., $\theta_{2}$ is independent of $\overline{\mathfrak{F}}_{\text {max }}$, and we can thus assume that $\theta_{1}>\theta_{2}+2 \kappa$. By pairwise-orthogonality, there exists $y \in P \times Q$ such that $\mathfrak{g}_{F}(y)=y_{F}$ for all $[F] \in \overline{\mathfrak{F}}_{\max }^{\prime}$ and $\mathfrak{g}_{Q}(y)=y_{Q}$. We now choose $\theta$ so that $\mathrm{d}_{\widehat{\mathcal{C}} W}\left(\pi_{W}(y), b_{W}\right) \leqslant \theta$ for all $[W] \in \mathfrak{F}$. 
First, if $W$ is parallel to a proper subcomplex of some $[F] \in \overline{\mathfrak{F}}_{\max }^{\prime}$, then any $\theta \geqslant \theta_{2}$ suffices.

Second, suppose that some $[F] \in \overline{\mathfrak{F}}_{\text {max }}^{\prime}$ is parallel to a proper subcomplex of $W$. Suppose $\mathrm{d}_{\widehat{\mathcal{C}} W}\left(b_{W}, \pi_{W}(F)\right) \leqslant \kappa+2 \theta_{1}$. Notice that $\pi_{W}(y)=\pi_{W}\left(y_{F}\right)$ because $F$ is parallel to a subcomplex of $W$, and hence $\mathrm{d}_{\widehat{\mathcal{C}} W}\left(b_{W}, \pi_{W}\left(y_{F}\right)\right)=\mathrm{d}_{\widehat{C} W}\left(b_{W}, \pi_{W}(y)\right) \leqslant 2 \kappa+2 \theta_{1}$. Hence suppose $\mathrm{d}_{\widehat{\mathcal{C}} W}\left(b_{W}, \pi_{W}(F)\right)>\kappa+2 \theta_{1}$, so $\operatorname{diam}_{\widehat{\mathcal{C}} F}\left(b_{F} \cup \rho_{F}^{W}\left(b_{W}\right)\right) \leqslant \kappa$. Since, up to parallelism, $W$ properly contains $F$ and $[F] \in \overline{\mathfrak{F}}_{\max }$, we can conclude that if $W \neq \mathcal{X}$ then we have $\mathrm{d}_{\widehat{\mathcal{C}} W}\left(b_{W}, \pi_{W}\left(x_{0}\right)\right) \leqslant \theta_{1}$, while the same inequality also holds in the case $W=\mathcal{X}$ because of our choice of $x_{0}$. Hence $\mathrm{d}_{\widehat{\mathcal{C}} W}\left(\pi_{W}\left(x_{0}\right), \pi_{W}(F)\right)>\kappa+\theta_{1}$. By Proposition 9.4, and the fact that a hierarchy path joining $\mathfrak{g}_{W}\left(x_{0}\right)$ to a point in $W$ projecting to $b_{W}$ cannot pass through an element of $[F]$ (because $\mathrm{d}_{\widehat{\mathcal{C}} W}\left(\pi_{W}\left(x_{0}\right), \pi_{W}(F)\right)>\kappa+\theta_{1} \geqslant \mathrm{~d}_{\widehat{\mathcal{C}} W}\left(\pi_{W}\left(x_{0}\right), b_{W}\right)$ ), we have $\mathrm{d}_{\widehat{\mathcal{C}} F}\left(\pi_{F}\left(x_{0}\right), \rho_{F}^{W}\left(b_{W}\right)\right) \leqslant 4 \xi+10$. Hence $\mathrm{d}_{\widehat{\mathcal{C}} F}\left(\pi_{F}\left(x_{0}\right), b_{F}\right) \leqslant 4 \xi+10+\kappa$, which contradicts $F \in \overline{\mathfrak{F}}_{\text {max }}$, so that this case does not arise.

Third, suppose that $[W]$ is transverse to some $[F] \in \overline{\mathfrak{F}}_{\text {max }}^{\prime}$. If $\mathrm{d}_{\widehat{\mathcal{C}} F}\left(\pi_{F}(y), \pi_{F}(W)\right)>\kappa+\theta_{2}$, then by consistency of $\vec{b}$, Proposition 12.2, and the fact $\pi_{W}(y) \in \pi_{W}(F)$ (since $y$ lies in a parallel copy of $F$ ), we have $\mathrm{d}_{\widehat{\mathcal{C}} W}\left(\pi_{W}(y), b_{W}\right) \leqslant \kappa$. Hence, suppose $\mathrm{d}_{\widehat{\mathcal{C}} F}\left(\pi_{F}(y), \pi_{F}(W)\right) \leqslant$ $\kappa+\theta_{2}$. In this case $\mathrm{d}_{\hat{\mathcal{C}} F}\left(\pi_{F}\left(x_{0}\right), \pi_{F}(W)\right)>\theta_{1}-\theta_{2}-\kappa$, whence $\mathrm{d}_{\widehat{\mathcal{C}} W}\left(\pi_{W}\left(x_{0}\right), \pi_{W}(F)\right) \leqslant \kappa$.

Now, since $y$ belongs to (a parallel copy of) $F$, we have $\mathrm{d}_{\widehat{\mathcal{C}} W}\left(\pi_{W}(y), \pi_{W}(F)\right)=0$. We now wish to argue that the inequality $\mathrm{d}_{\widehat{\mathcal{C}} W}\left(\pi_{W}(y), b_{W}\right) \geqslant \theta_{1}+\xi+2+\kappa$ is impossible, thus concluding the proof in this case. In fact, if it holds, then

$$
\mathrm{d}_{\widehat{\mathcal{C}} W}\left(\pi_{W}\left(x_{0}\right), b_{W}\right) \geqslant \mathrm{d}_{\widehat{\mathcal{C}} W}\left(\pi_{W}(y), b_{W}\right)-\operatorname{diam}\left(\pi_{W}(F)\right)-\mathrm{d}_{\widehat{\mathcal{C}} W}\left(\pi_{W}(F), \pi_{W}\left(x_{0}\right)\right) \geqslant \theta_{1} .
$$

Hence $W$ is contained in some $W^{\prime}$ with $\left[W^{\prime}\right] \in \overline{\mathfrak{F}}_{\text {max }}$. Notice that $\left[W^{\prime}\right]$ must be transverse to $[F]$, since $F$ cannot be parallel into $W$ nor vice versa, by maximality of $F$ and $W^{\prime}$, and since, if $\left[W^{\prime}\right]$ was orthogonal to $[F]$, then $[W]$ would be as well.

Note $\mathrm{d}_{\widehat{\mathcal{C}} F}\left(\pi_{F}(y), \pi_{F}\left(W^{\prime}\right)\right) \leqslant \kappa$, since this holds for $W$. Thus $\mathrm{d}_{\widehat{\mathcal{C}} W^{\prime}}\left(\pi_{W^{\prime}}\left(x_{0}\right), \pi_{W^{\prime}}(F)\right) \leqslant \kappa$, by an argument above. But then, by Claim 1, the closest parallel copy of $W^{\prime}$ to $x$ is closer than the closest parallel copy of $F$, in contradiction with $[F] \in \overline{\mathfrak{F}}_{\max }^{\prime}$.

The only case left is when $W$ is orthogonal to all $[F] \in \overline{\mathfrak{F}}_{\max }^{\prime}$, which implies that $W$ is, up to parallelism, contained in $Q$. In this case, $\pi_{W}(y)=\pi_{W}\left(\mathfrak{g}_{Q}\left(y_{Q}\right)\right)=\pi_{W}\left(y_{Q}\right)$, whence we are done by the choice of $y_{Q}$.

Remark 12.5. Let $\kappa \geqslant \kappa_{0}$ and let $\vec{b}$ be a $\kappa$-consistent tuple. Theorem 12.4 yields a constant $\theta$ and a 0 -cube $x \in \mathcal{X}^{(0)}$ such that $\mathrm{d}_{\widehat{\mathcal{C}} F}\left(x, b_{F}\right) \leqslant \theta$ for all $F \in \mathfrak{F}$. By Theorem 9.1, any other 0 -cube $y$ with this property is at a uniformly bounded distance - depending on $\kappa$ and $\xi$ from $x$. The definition of a factor system implies that $\mathcal{X}$ is uniformly locally finite, so the number of such 0 -cubes $y$ is bounded by a constant depending only on $\kappa, \xi$, and the growth function of $\mathcal{X}^{(0)}$.

\section{Part 3. Hierarchically hyperbolic spaces}

\section{QuASI-BOXES IN HIERARCHICALLY HYPERBOLIC SPACES}

In this section, we work in a level of generality which includes both mapping class groups and each CAT(0) cube complex $\mathcal{X}$ with a factor system $\mathfrak{F}$.

Definition 13.1 (Hierarchically hyperbolic space). The metric space $\left(\mathcal{X}, \mathrm{d}_{\mathcal{X}}\right)$ is a hierarchically hyperbolic space if there exists $\delta \geqslant 0$, an index set $\mathfrak{S}$, and a set $\{\widehat{\mathcal{C}} W \mid W \in \mathfrak{S}\}$ of $\delta$-hyperbolic spaces, such that the following conditions are satisfied:

(1) (Projections.) There is a set $\left\{\pi_{W}: \mathcal{X} \rightarrow 2^{\hat{\mathcal{C}} W} \mid W \in \mathfrak{S}\right\}$ of projections sending points in $\mathcal{X}$ to sets of diameter bounded by some $\xi \geqslant 0$ in the various $\hat{\mathcal{C}} W \in \mathfrak{S}$. 
(2) (Nesting.) $\mathfrak{S}$ is equipped with a partial order $\sqsubseteq$, and either $\mathfrak{S}=\varnothing$ or $\mathfrak{S}$ contains a unique $\sqsubseteq$-maximal element; when $V \sqsubseteq W$, we say $V$ is nested in $W$. We require that $W \sqsubseteq W$ for all $W \in \mathfrak{S}$. For each $W \in \mathfrak{S}$, we denote by $\mathfrak{S}_{W}$ the set of $V \in \mathfrak{S}$ such that $V \sqsubseteq W$. Moreover, for all $V, W \in \mathfrak{S}$ with $V$ properly nested into $W$ there is a specified subset $\rho_{W}^{V} \subset \widehat{\mathcal{C}} W$ with $\operatorname{diam}_{\widehat{\mathcal{C}} W}\left(\rho_{W}^{V}\right) \leqslant \xi$. There is also a projection $\rho_{V}^{W}: \hat{\mathcal{C}} W \rightarrow 2^{\widehat{\mathcal{C}} V}$. (The similarity in notation is justified by viewing $\rho_{W}^{V}$ as a coarsely constant map $\widehat{\mathcal{C}} V \rightarrow 2^{\hat{\mathcal{C}} W}$.)

(3) (Orthogonality.) $\mathfrak{S}$ has a symmetric and anti-reflexive relation called orthogonality: we write $V \perp W$ when $V, W$ are orthogonal. Also, whenever $V \sqsubseteq W$ and $W \perp U$, we require that $V \perp U$. Finally, we require that for each $T \in \mathfrak{S}$ and each $U \in \mathfrak{S}_{T}$ for which $\left\{V \in \mathfrak{S}_{T} \mid V \perp U\right\} \neq \varnothing$, there exists $W \in \mathfrak{S}_{T}-\{T\}$, so that whenever $V \perp U$ and $V \sqsubseteq T$, we have $V \sqsubseteq W$. Finally, if $V \perp W$, then $V, W$ are not $\sqsubseteq$-comparable.

(4) (Transversality and consistency.) If $V, W \in \mathfrak{S}$ are not orthogonal and neither is nested in the other, then we say $V, W$ are transverse, denoted $V \pitchfork W$. There exists $\kappa_{0} \geqslant 0$ such that if $V \pitchfork W$, then there are sets $\rho_{W}^{V} \subseteq \widehat{\mathcal{C}} W$ and $\rho_{V}^{W} \subseteq \widehat{\mathcal{C}} V$ each of diameter at most $\xi$ and satisfying:

$$
\min \left\{\mathrm{d}_{\widehat{\mathcal{C}} W}\left(\pi_{W}(x), \rho_{W}^{V}\right), \mathrm{d}_{\widehat{\mathcal{C}} V}\left(\pi_{V}(x), \rho_{V}^{W}\right)\right\} \leqslant \kappa_{0}
$$

for all $x \in \mathcal{X}$; alternatively, in the case $V \sqsubseteq W$, then for all $x \in \mathcal{X}$ we have:

$$
\min \left\{\mathrm{d}_{\widehat{\mathcal{C}} W}\left(\pi_{W}(x), \rho_{W}^{V}\right), \operatorname{diam}_{\hat{\mathcal{C}} V}\left(\pi_{V}(x) \cup \rho_{V}^{W}\left(\pi_{W}(x)\right)\right)\right\} \leqslant \kappa_{0} .
$$

Suppose that: either $U \subsetneq V$ or $U \pitchfork V$, and either $U \subsetneq W$ or $U \pitchfork W$. Then we have: if $V \pitchfork W$, then

$$
\min \left\{\mathrm{d}_{\widehat{\mathcal{C}} W}\left(\rho_{W}^{U}, \rho_{W}^{V}\right), \mathrm{d}_{\widehat{\mathcal{C}} V}\left(\rho_{V}^{U}, \rho_{V}^{W}\right)\right\} \leqslant \kappa_{0}
$$

and if $V \subsetneq W$, then

$$
\min \left\{\mathrm{d}_{\widehat{\mathcal{C}} W}\left(\rho_{W}^{U}, \rho_{W}^{V}\right), \operatorname{diam}_{\widehat{\mathcal{C}} V}\left(\rho_{V}^{U} \cup \rho_{V}^{W}\left(\rho_{W}^{U}\right)\right)\right\} \leqslant \kappa_{0} .
$$

Finally, if $V \sqsubseteq U$ or $U \perp V$, then $\mathrm{d}_{\widehat{\mathcal{C}} W}\left(\rho_{W}^{U}, \rho_{W}^{V}\right) \leqslant \kappa_{0}$ whenever $W \in \mathfrak{S}-\{U, V\}$ satisfies either $V \sqsubseteq W$ or $V \pitchfork W$ and either $U \sqsubseteq W$ or $U \pitchfork W$.

(5) (Finite complexity.) There exists $n \geqslant 0$, the complexity of $\mathcal{X}$ (with respect to $\mathfrak{S}$ ), so that any sequence $\left(U_{i}\right)$ with $U_{i}$ properly nested into $U_{i+1}$ has length at most $n$.

(6) (Distance formula.) There exists $s_{0} \geqslant \xi$ such that for all $s \geqslant s_{0}$ there exist constants $K, C$ such that for all $x, x^{\prime} \in \mathcal{X}$,

$$
\mathrm{d}_{\mathcal{X}}\left(x, x^{\prime}\right)=_{(K, C)} \sum_{W \in \mathfrak{S}}\left\{\left\{\mathrm{d}_{\widehat{\mathcal{C}} W}\left(\pi_{W}(x), \pi_{W}\left(x^{\prime}\right)\right)\right\}_{s} .\right.
$$

We often write $\sigma_{\mathcal{X}, s}\left(x, x^{\prime}\right)$ to denote the right-hand side of Item (6); more generally, given $W \in \mathfrak{S}$, we denote by $\sigma_{W, s}\left(x, x^{\prime}\right)$ the corresponding sum taken over $\mathfrak{S}_{W}$.

(7) (Large links.) There exists $\lambda \geqslant 1$ such that the following holds. Let $W \in \mathfrak{S}$ and let $x, x^{\prime} \in \mathcal{X}$. Let $N=\lambda_{\mathrm{d}_{\widehat{\mathcal{C}} W}}\left(\pi_{W}(x), \pi_{W}\left(x^{\prime}\right)\right)+\lambda$. Then there exists $\left\{T_{i}\right\}_{i=1, \ldots,\lfloor N\rfloor} \subseteq$ $\mathfrak{S}_{W}-\{W\}$ such that for all $T \in \mathfrak{S}_{W}-\{W\}$, either $T \in \mathfrak{S}_{T_{i}}$ for some $i$, or $\mathrm{d}_{\widehat{\mathcal{C}} T}\left(\pi_{T}(x), \pi_{T}\left(x^{\prime}\right)\right)<s_{0}$. Also, $\mathrm{d}_{\widehat{\mathcal{C}} W}\left(\pi_{W}(x), \rho_{W}^{T_{i}}\right) \leqslant N$ for each $i$.

(8) (Bounded geodesic image.) For all $W \in \mathfrak{S}$, all $V \in \mathfrak{S}_{W}-\{W\}$, and all geodesics $\gamma$ of $\widehat{\mathcal{C}} W$, either $\operatorname{diam}_{\widehat{\mathcal{C}} V}\left(\rho_{V}^{W}(\gamma)\right) \leqslant B$ or $\gamma \cap \mathcal{N}_{E}\left(\rho_{W}^{V}\right) \neq \varnothing$ for some uniform $B, E$.

(9) (Realization.) For each $\kappa$ there exists $\theta_{e}, \theta_{u}$ such that the following holds. Let $\vec{b} \in \prod_{W \in \mathfrak{S}} 2^{\hat{\mathcal{C}} W}$ have each coordinate correspond to a subset of $\widehat{\mathcal{C}} W$ of diameter at most $\kappa$; for each $W$, let $b_{W}$ denote the $\widehat{\mathcal{C}} W$-coordinate of $\vec{b}$. Suppose that whenever $V \pitchfork W$ we have

$$
\min \left\{\mathrm{d}_{\widehat{\mathcal{C}} W}\left(b_{W}, \rho_{W}^{V}\right), \mathrm{d}_{\widehat{\mathcal{C}} V}\left(b_{V}, \rho_{V}^{W}\right)\right\} \leqslant \kappa
$$


and whenever $V \sqsubseteq W$ we have

$$
\min \left\{\mathrm{d}_{\widehat{\mathcal{C}} W}\left(b_{W}, \rho_{W}^{V}\right), \operatorname{diam}_{\widehat{\mathcal{C}} V}\left(b_{V} \cup \rho_{V}^{W}\left(b_{W}\right)\right)\right\} \leqslant \kappa .
$$

Then there the set of all $x \in \mathcal{X}$ so that $\mathrm{d}_{\widehat{\mathcal{C}} W}\left(b_{W}, \pi_{W}(x)\right) \leqslant \theta_{e}$ for all $\widehat{\mathcal{C}} W \in \mathfrak{S}$ is non-empty and has diameter at most $\theta_{u}$. (A tuple $\vec{b}$ satisfying the inequalities above is called $\kappa$-consistent.)

(10) (Hierarchy paths.) There exists $D \geqslant 0$ so that any pair of points in $\mathcal{X}$ can be joined by a $(D, D)$-quasi-geodesic $\gamma$ with the property that, for each $W \in \mathfrak{S}$, the projection $\pi_{W}(\gamma)$ is at Hausdorff distance at most $D$ from any geodesic connecting $\pi_{W}(x)$ to $\pi_{W}(y)$. We call such quasi-geodesics hierarchy paths.

We say that the metric spaces $\left\{\mathcal{X}_{i}\right\}$ are uniformly hierarchically hyperbolic if each $\mathcal{X}_{i}$ satisfies the axioms above and all constants can be chosen uniformly.

Observe that a space $\mathcal{X}$ is hierarchically hyperbolic with respect to $\mathfrak{S}=\varnothing$, i.e., hierarchically hyperbolic of complexity 0 , if and only if $\mathcal{X}$ is bounded. Similarly, $\mathcal{X}$ is hierarchically hyperbolic of complexity 1 , with respect to $\mathfrak{S}=\{\mathcal{X}\}$, if and only if $\mathcal{X}$ is hyperbolic.

Remark 13.2 (Cube complexes with factor systems are hierarchically hyperbolic spaces). In the case where $\mathcal{X}$ is a $\operatorname{CAT}(0)$ cube complex with a factor system, we take $\mathfrak{S}$ to be a subset of the factor system containing exactly one element from each parallelism class. We take $\{\widehat{\mathcal{C}} W \mid W \in \mathfrak{S}\}$ to be the set of factored contact graphs of elements of $\mathfrak{S}$. All of the properties required by Definition 13.1 are satisfied: given $U, V \in \mathfrak{S}$, we have $U \sqsubseteq V$ if and only if $U$ is parallel into $V$; orthogonality is defined as in Section 10 . The last condition on orthogonality is satisfied since each element of a factor system is either unique in its parallelism class or is contained in a hyperplane. The first two inequalities in Item (4) follow from Proposition 10.7 the second two follow from the first two since each $\rho_{V}^{U}$ is the image of the convex subcomplex $U \in \mathfrak{S}$ under the projection $\pi_{V}$. Finiteness of complexity follows from the definition of a factor system (specifically, uniform local finiteness of the family of factors). Item (6) is provided by Theorem 9.1, Item (7) by Proposition 9.4. Item (8) is Proposition 8.20, Item (9) is Theorem 12.4, and Item $\sqrt{10}$ ) is Proposition 8.23. In order to ensure that nesting and orthogonality are mutually exclusive, we require that elements of the factor system $\mathfrak{S}$ are not single points.

Remark 13.3. The Bounded Geodesic Image constant $E$ from Definition 13.1 can be taken to be 1 for cube complexes with factor systems, as well as for the mapping class group. However, we allow an arbitrary (fixed) constant in the general definition for greater flexibility.

Remark 13.4. In the case of a factor system, the constant in Item (1) of Definition 13.1 is 1, since points of $\mathcal{X}$ project to cliques in each factored contact graph. The constant in Item (2) is $\xi+2$, where $\xi$ is the constant from Definition 8.1. For simplicity, in Definition 13.1, we use a single constant to fulfill both of these roles.

13.1. Product regions and standard boxes. Let $\mathcal{X}$ be a hierarchically hyperbolic space and let $U \in \mathfrak{S}$. With $\kappa_{0}$ as in Definition 13.1. we say that the tuple $\vec{a}_{\sqsubseteq} \in \prod_{W \sqsubseteq U} 2^{\widehat{\mathcal{C}} W}$ or $\vec{a}_{\perp} \in \prod_{W \perp U} 2^{\hat{\mathcal{C}} W}$, is $\kappa_{0}$-consistent if the inequalities from Item (4) are satisfied for each pair of coordinates $a_{W}$ and $a_{V}$ in the tuple with the relevant $\pi_{W}(x)$ term replaced by $a_{W}$ (and similarly for $V$ ).

Let $\vec{a}_{\sqsubseteq}$ and $\vec{a}_{\perp}$ be $\kappa_{0}$-consistent. Let $\vec{a} \in \prod_{\hat{\mathcal{C}} W \in \mathfrak{S}} 2^{\hat{\mathcal{C}} W}$ be the tuple with $a_{W}=\rho_{W}^{U}$ for each $W \pitchfork U$ or $U \sqsubseteq W$, and so that $a_{W}$ otherwise agrees with the relevant coordinate of $\vec{a}_{\sqsubseteq}$ or $\vec{a}_{\perp}$, depending on whether $W \sqsubseteq U$ or $W \perp U$. We claim that $\vec{a}$ is $\kappa_{0}$-consistent. Indeed, all inequalities involving some $W \pitchfork U$ or $U \sqsubseteq W$ are satisfied because of the last three inequalities in Definition 13.1. (4). Otherwise, if $W \sqsubseteq U$ or $W \perp U$, then, if $V \sqsubseteq W$, we have that $V, W$ are 
either both nested into or both orthogonal to $U$, and in both cases the consistency inequality holds by assumption.

Item 9 of Definition 13.1 (realization) combines with the above discussion to yield a natural coarsely defined map $\phi_{U}: E_{U} \times F_{U} \rightarrow \mathcal{X}$, where $E_{U}$ (resp. $F_{U}$ ) is the set of consistent elements of $\prod_{W \perp U} 2^{\hat{\mathcal{C}} W}$ (resp. $\prod_{W \sqsubseteq U} 2^{\hat{\mathcal{C}} W}$ ).

Remark 13.5. When $\mathcal{X}$ is a cube complex with a factor system $\mathfrak{S}$, the space $F_{U}$ can be taken to be the subcomplex $U \in \mathfrak{S}$, we can take $E_{U}$ to be the complex provided by Lemma 2.4 . and $\phi_{U}$ to be the inclusion, which is a cubical isometric embedding.

We will denote $P_{U}=\phi_{U}\left(E_{U} \times F_{U}\right)$. In this context we define a gate, $\mathfrak{g}_{P_{U}}: \mathcal{X} \rightarrow P_{U}$, in the following way. Given $x \in \mathcal{X}$, we let $\mathfrak{g}_{P_{U}}(x)$ be a point that coarsely realizes the coordinates $a_{W}=\pi_{W}(x)$ for $W$ nested into or orthogonal to $U$ and $\pi_{W}(x)=\rho_{W}^{U}$ for $W \pitchfork U$ or $U \sqsubseteq W$.

By Definition 13.1.(6) (distance formula), for each fixed $U$ the subspaces $\phi_{U}\left(E_{U} \times\left\{b_{\sqsubseteq}\right\}\right.$ ) of $\mathcal{X}$ are pairwise uniformly quasi-isometric, and similarly for $F_{U}$. Abusing notation slightly, we sometimes regard $E_{U}$ as a metric space by identifying it with some $\phi_{U}\left(E_{U} \times\left\{b_{\sqsubseteq}\right\}\right)$, and similarly for $F_{U}$. Moreover, $E_{U}$ is a hierarchically hyperbolic space, with respect to $\{V \in \mathfrak{S}: V \perp U\} \cup\{W\}$, where $W$ is some non-maximal element of $\mathfrak{S}$ into which each element orthogonal to $U$ is nested; and, similarly, $F_{U}$ is a hierarchically hyperbolic space with respect to $\mathfrak{S}_{U}$. It is straightforward to check that $\left\{E_{U}\right\}_{U \in \mathfrak{S}}$ are uniformly hierarchically hyperbolic with respect to the index set described above, with constants depending only on the constants for $\mathcal{X}$.

Remark 13.6 (How to induct on complexity). Observe that if $U$ is not $\sqsubseteq$-maximal, then any $\sqsubseteq$-chain in $\mathfrak{S}_{U}$ is strictly contained in a $\sqsubseteq$-chain of $\mathfrak{S}$, so that the complexity of $F_{U}$ is strictly less than that of $\mathcal{X}$. Moreover, by definition, there exists $W \in \mathfrak{S}$, not $\sqsubseteq$-maximal, such that each $V$ with $V \perp U$ satisfies $V \sqsubseteq W$. Hence $E_{U}$ has complexity strictly less than that of $\mathcal{X}$. We will make use of these observations when inducting on complexity in the proof of Theorem 13.11.

A standard $1-b o x$ is a hierarchy path $I \rightarrow F_{U}$ where $U \in \mathfrak{S}$. We then inductively define a standard $n$-box to be a map of the kind $\iota:\left(B \times I \subseteq \mathbb{R}^{n-1} \times \mathbb{R}\right) \rightarrow \mathcal{X}$, with $\iota(b, t)=$ $\phi_{U}\left(\iota_{1}(b), \gamma(t)\right)$ where $\iota_{1}: B \rightarrow E_{U}$ is a standard box and $\gamma: I \rightarrow F_{U}$ is a hierarchy path. It is straightforward to show inductively that a standard box is a quasi-isometric embedding.

13.2. Results. The goal of this section is to establish the following three theorems:

Theorem 13.7. Let $\mathcal{X}$ be a hierarchically hyperbolic space. Then for every $n \in \mathbb{N}$ and every $K, C, R_{0}, \epsilon_{0}$ the following holds. There exists $R_{1}$ so that for any ball $B \subseteq \mathbb{R}^{n}$ of radius at least $R_{1}$ and $f: B \rightarrow \mathcal{X}$ a $(K, C)$-quasi-Lipschitz map, there is a ball $B^{\prime} \subseteq B$ of radius $R^{\prime} \geqslant R_{0}$ such that $f\left(B^{\prime}\right)$ lies inside the $\epsilon_{0} R^{\prime}$-neighborhood of a standard box.

Remark. In Theorem 13.7, we do not require $B, B^{\prime}$ to be centered at the same point in $\mathbb{R}^{n}$.

Theorem 13.8. Let $\mathcal{X}$ be a hierarchically hyperbolic space. Then for every simply connected nilpotent Lie group $\mathcal{N}$, with a left-invariant Riemannian metric, and every $K, C$ there exists $R$ with the following property. For every $(K, C)$-quasi-Lipschitz map $f: B \rightarrow \mathcal{X}$ from a ball in $\mathcal{N}$ into $\mathcal{X}$ and for every $h \in \mathcal{N}$ we have $\operatorname{diam}(f(B \cap h[\mathcal{N}, \mathcal{N}])) \leqslant R$. In particular, any finitely generated nilpotent group which quasi-isometrically embeds into $\mathcal{X}$ is virtually abelian.

Theorem 13.9. Let $\mathcal{X}$ be a hierarchically hyperbolic space with respect to a set $\mathfrak{S}$. If there exists a quasi-isometric embedding $f: \mathbb{R}^{n} \rightarrow \mathcal{X}$ then $n$ is bounded by the maximal cardinality of a set of pairwise-orthogonal elements of $\mathfrak{S}$, and in particular by the complexity of $\mathcal{X}$. 
Notation 13.10. Let $\omega$ be a non-principal ultrafilter, which will be arbitrary, but fixed for the rest of the paper. For a sequences of spaces $\left(X_{m}\right)$, we denote their ultralimit $\lim _{\omega} X_{m}$ by $\boldsymbol{X}$, similarly for sequences of maps $\lim _{\omega} f_{m}=\boldsymbol{f}$, etc. For scaling constants, we will have sequences of positive real numbers $\left(r_{m}\right)$; when comparing two such sequences we write $\left(r_{m}\right)<\left(r_{m}^{\prime}\right)$ if $r_{m}<r_{m}^{\prime}$ for $\omega$-a.e. $m$. Also, we write $\left(r_{m}\right) \ll\left(r_{m}^{\prime}\right)$ if $\lim _{\omega} r_{m}^{\prime} / r_{m}=\infty$.

Each of the above theorems follows from:

Theorem 13.11. Let $\left\{\mathcal{X}_{m}\right\}$ be uniformly hierarchically hyperbolic spaces and let $\mathcal{N}$ be a simply connected nilpotent Lie group endowed with a left-invariant Riemannian metric. Let $\left(f_{m}: B_{m} \rightarrow \mathcal{X}_{m}\right)$ be a sequence of quasi-Lipschitz maps with uniformly bounded constants, where $B_{m} \subseteq \mathcal{N}$ is a ball of radius $r_{m}^{1}$. Then:

(1) For every ultralimit $\mathcal{X}$ of $\left(\mathcal{X}_{m}\right)$ and corresponding ultralimit map $\boldsymbol{f}: \boldsymbol{B} \rightarrow \mathcal{X}$, the map $\boldsymbol{f}$ is constant along ultralimits of cosets of $[\mathcal{N}, \mathcal{N}]$.

(2) Suppose that $\mathcal{N}$ is abelian and let $\left(r_{m}^{0}\right)$ satisfy $\left(r_{m}^{0}\right) \ll\left(r_{m}^{1}\right)$. Then there exist sequences $\left(p_{m}\right)$ and $\left(r_{m}\right)$ with $\left(r_{m}^{0}\right) \ll\left(r_{m}\right) \leqslant\left(r_{m}^{1}\right)$ and $B\left(p_{m}, r_{m}\right) \subseteq B_{m}$ so that the following holds. Let $\mathcal{X}$ be the ultralimit of $\left(\mathcal{X}_{m}\right)$ with scaling factor $\left(r_{m}\right)$ and observation point $\left(f\left(p_{m}\right)\right)$ and let $\boldsymbol{B}$ be the ultralimit of the sequence $B\left(p_{m}, r_{m}\right)$ with observation point $\left(p_{m}\right)$ and scaling factors $\left(r_{m}\right)$. Then there exists an ultralimit $\boldsymbol{F}$ of standard boxes so that the ultralimit map $\boldsymbol{f}: \boldsymbol{B} \rightarrow \mathcal{X}$ satisfies $\boldsymbol{f}(\boldsymbol{B}) \subseteq \boldsymbol{F}$.

We first deduce the other theorems from Theorem 13.11 .

Proof of Theorem 13.7. Fix $n, K, C, \epsilon_{0}, R_{0}>0$. If the statement was false then there would exist a sequence of maps $f_{m}: B_{m} \rightarrow \mathcal{X}$ where

- each $B_{m}$ is a ball of radius $r_{m}^{1}$ about $0 \in \mathbb{R}^{n}$, each $f_{m}$ is $(K, C)$-quasi-Lipschitz and $r_{m}^{1} \rightarrow \infty$

- no ball $B^{\prime} \subseteq B_{m}$ of radius $R^{\prime} \geqslant R_{0}$ is so that $f_{m}\left(B^{\prime}\right)$ is contained in the $\epsilon_{0} R^{\prime}-$ neighborhood of a standard box.

In the notation of Theorem 13.11.(2), if $\left(p_{m}\right)$ is the observation point of $\boldsymbol{B}$, then it is readily checked that, $\omega$-a.e., we have $f_{m}\left(B\left(p_{m}, r_{m}\right)\right) \subseteq \mathcal{N}_{\epsilon_{0} r_{m}}\left(F_{m}\right)$, where $\boldsymbol{r}=\left(r_{m}\right), \boldsymbol{F}=$ $\left(F_{m}\right)$. By the proof of Theorem 13.11. (2), we have that $\mathrm{d}_{\mathbb{R}^{n}}\left(p_{m}, 0\right) \leqslant r_{m}^{1} / 2$. Letting $s_{m}=$ $\min \left\{r_{m}, r_{m}^{1} / 2\right\}$, and recalling that $\lim _{\omega} r_{m}^{1}=\lim _{\omega} r^{m}=+\infty$, we have $s_{m} \geqslant R_{0} \omega$-a.e.; thus for sufficiently large $m$, taking $B^{\prime}=B\left(p_{m}, s_{m}\right)$ contradicts the second item above.

Proof of Theorem 13.8. This follows directly from Theorem 13.11.(1). The statement about nilpotent groups follows from the well-known fact that every finitely generated nilpotent group is virtually a lattice in a simply-connected nilpotent Lie group. Mal49.

Proof of Theorem 13.9. Using Theorem 13.11. (2), we see that some rescaled ultralimit $\boldsymbol{f}$ of $f$ maps an asymptotic cone of $\mathbb{R}^{n}$, which is itself a copy of $\mathbb{R}^{n}$, into a rescaled ultralimit of standard boxes, whence the claim follows.

13.3. Proof of Theorem 13.11. We now complete the proofs of Theorems 13.7, 13.8, and 13.9 by proving Theorem 13.11. Throughout, $\mathcal{X}$ is a hierarchically hyperbolic space; below we use the notation from Definition 13.1.

Fix $W \in \mathfrak{S}$ and let $\gamma: I \rightarrow F_{W}$ be a hierarchy path: we now define the "projection" from $\mathcal{X}$ to $\gamma$. Roughly speaking, we map $x \in \mathcal{X}$ to any point in $\gamma$ that minimizes the $\widehat{\mathcal{C}} W$-distance from $x$. Let $\beta=\pi_{W}(\gamma(I))$ and let $c_{\beta}: \widehat{\mathcal{C}} W \rightarrow \beta$ be closest-point projection. Define a map $q_{\gamma}: \mathcal{X} \rightarrow \gamma$ as follows: for each $z \in \beta$, let $t(z)$ be an arbitrary point of $\gamma$ so that $\pi_{W}(t(z))=z$, and then, for each $x \in \mathcal{X}$, let $q_{\gamma}(x)=t\left(c_{\beta}\left(\pi_{W}(x)\right)\right)$.

Let $P_{W}$ denote the image in $\mathcal{X}$ of the restriction of $\phi_{W}$ to $E_{W} \times(\operatorname{im} \gamma)$. 
Lemmas 13.12 and 13.13 accomplish in the hierarchically hyperbolic setting an analog of a result in the mapping class group proven in [BM08, Theorems 3.4 and 3.5]; note that in BM08 the results are formulated in the asymptotic cone, rather than in the original space.

Lemma 13.12. Let $(\mathcal{X}, \mathrm{d})$ be a hierarchically hyperbolic space. There exists a constant $\ell \geqslant 1$ depending only on the constants from Definition 13.1 so that the following holds. Suppose that $\gamma: I \rightarrow F_{W}$ is a hierarchy path connecting $x$ to $y$ and let $M=\sup _{U \in \mathfrak{S}_{W}-\{W\}} \sigma_{U, s_{0}}(x, y)$. Let $z \in \mathcal{X}$ and let $R=\mathrm{d}_{\mathcal{X}}\left(z, P_{W}\right)$. Then

$$
\operatorname{diam}_{\mathcal{X}}\left(q_{\gamma}(B(z, R / \ell))\right) \leqslant \ell M+\ell .
$$

Proof. For a suitable constant $\ell$, we will prove the following: given $v, w \in \mathcal{X}$, if $\mathrm{d}\left(q_{\gamma}(v), q_{\gamma}(w)\right)>$ $\ell M+\ell$, then any hierarchy path $\alpha$ from $v$ to $w$ intersects the $\ell M$-neighborhood of $P_{W}$; then the the lemma will follow by increasing $\ell$.

First, fix a hierarchy path $\alpha$ from $v$ to $w$. For $\ell$ large enough, the $\delta$-hyperbolicity of $\widehat{\mathcal{C}} W$ and the fact that $\alpha$ is a hierarchy path guarantee the existence of points $t_{1}, t, t_{2}$, appearing in this order along $\alpha$, so that:

(1) $\mathrm{d}_{\widehat{\mathcal{C}} W}(t, \gamma) \leqslant 2 \delta+2 D$,

(2) $\mathrm{d}_{\widehat{c} W}\left(t, c_{\beta}\left(\pi_{W}(v)\right)\right), d_{\widehat{c} W}\left(t, c_{\beta}\left(\pi_{W}(w)\right)\right) \geqslant 100 \delta+100 D$,

(3) $\mathrm{d}_{\widehat{\mathcal{C}} W}\left(t, t_{i}\right)>10 \kappa_{0}$ for $i \in\{1,2\}$.

Using Definition 13.1. (9) (Realization) we pick any $t^{\prime} \in P_{W}$ whose $\widehat{\mathcal{C}} U$-coordinate is $\kappa^{-}$ close to that of $c_{\beta}(t)$ whenever $U \sqsubseteq W$ and whose $\widehat{\mathcal{C}} U$-coordinate is $\kappa$-close to that of $t$ whenever $U \perp W$.

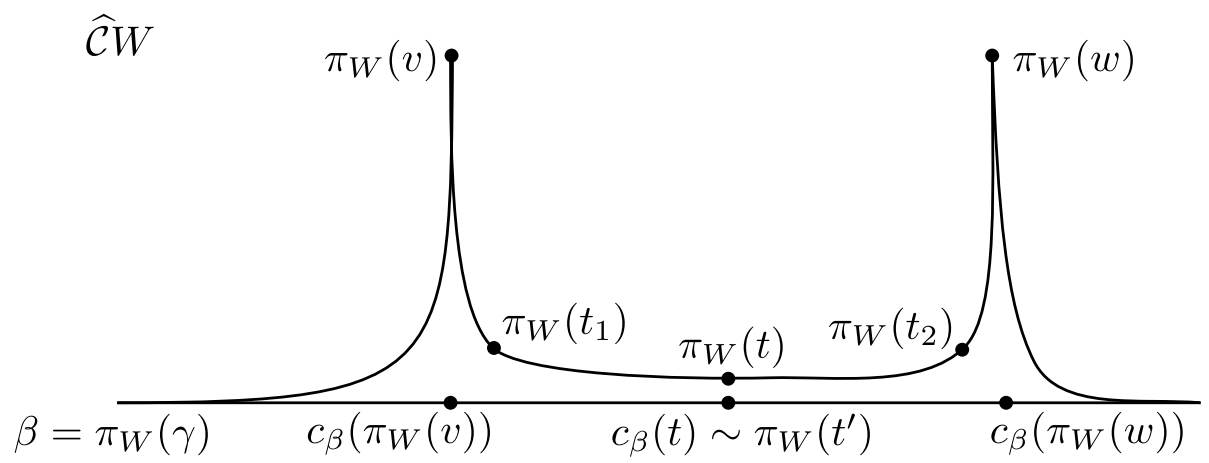

By choice of $t^{\prime}$ and taking the threshold in the distance formula larger than $\kappa$ we have that any term in the distance formula contributing to $\mathrm{d}_{\mathcal{X}}\left(t, t^{\prime}\right)$ comes from some $U \in \mathfrak{S}$ which is either nested in $W$ or transverse to it. We will first argue that the latter terms are uniformly bounded. This is because if $U \pitchfork W$ contributes to the distance formula with any threshold $\geqslant \kappa_{0}+\kappa$, we have $d_{\widehat{\mathcal{C}} U}\left(\rho_{U}^{W}, \pi_{U}(t)\right)>\kappa_{0}$ and hence $d_{\widehat{\mathcal{C}} W}\left(\rho_{W}^{U}, \pi_{W}(t)\right) \leqslant \kappa_{0}$ by the first consistency inequality (see Definition 13.1. (4) ). But then we also have $d_{\widehat{\mathcal{C}} W}\left(\rho_{W}^{U}, \pi_{W}\left(t_{i}\right)\right)>\kappa_{0}$ and hence again by the first consistency condition we have $d_{\widehat{\mathcal{C}} U}\left(\rho_{U}^{W}, \pi_{U}\left(t_{i}\right)\right) \leqslant \kappa_{0}$. However, since $\pi_{W} \circ \alpha$ is an unparameterized $(D, D)$-quasi-geodesic and $t$ lies between $t_{1}$ and $t_{2}$, there is a bound on $d_{\widehat{\mathcal{C}} U}\left(\rho_{U}^{W}, \pi_{U}(t)\right)$ in terms of $\kappa_{0}, D, \delta$ only; this is the uniform bound we wanted. From now on, we assume that the thresholds used in the distance formula exceed this bound. We now know that $\sigma_{W, s}\left(t, t^{\prime}\right)$ coarsely bounds $\mathrm{d} \mathcal{X}\left(t, t^{\prime}\right)$ for any sufficiently large threshold $s$.

By Definition 13.1. (7) (Large Link Lemma), any term that contributes to $\sigma_{W, s_{0}}\left(t, t^{\prime}\right)$ is either $W$ or an element of $\mathfrak{S}_{T_{i}}$, for at most $\lambda(2 \delta+2 D+1)$ elements $T_{i} \in \mathfrak{S}_{W}-\{W\}$, each within distance $\lambda(2 \delta+2 D+1)$ from $\pi_{W}(t)$, where $\lambda \geqslant 1$ is a uniform constant.

By Definition 13.1.(8) (Bounded Geodesic Image), applied to geodesics joining $\pi_{W}(v)$ to $\pi_{W}(x)$ and from $\pi_{W}(w)$ to $\pi_{W}(y)$ (or vice versa), we have $d_{\widehat{\mathcal{C}} F}(v, x), d_{\widehat{\mathcal{C}} F}(w, y) \leqslant B$ for any 
$F \in \mathfrak{S}_{T_{i}}$. Since $\pi_{F}(t)$ is $D$-close to a geodesic from $\pi_{F}(v)$ to $\pi_{F}(w)$ for $F \in \mathfrak{S}_{T_{i}}$ and similarly for $\pi_{F}\left(t^{\prime}\right)$ with respect to $\pi_{F}(v)$ and $\pi_{F}(w)$, we thus have

$$
\sum_{F \in \mathfrak{S}_{T_{i}}}\left\{\left\{d_{\widehat{\mathcal{C}} F}\left(t, t^{\prime}\right)\right\}_{s_{0}+2 D+2 B} \leqslant R \sum_{F \in \mathfrak{S}_{T_{i}}}\left\{\left\{d_{\widehat{\mathcal{C}} F}(x, y)\right\}_{s_{0}}\right.\right.
$$

where $R=\left(s_{0}+2 D+2 B\right) / s_{0}$. Hence

$\sigma_{W, s_{0}+2 D+2 B}\left(t, t^{\prime}\right) \leqslant \lambda(2 \delta+2 D+1)+\lambda(2 \delta+2 D+1) R \max _{i}\left\{\sum_{F \in \mathfrak{S}_{T_{i}}}\left\{d_{\widehat{\mathcal{C}} F}(x, y)\right\}_{s_{0}}\right\} \leqslant \ell^{\prime} M+\ell^{\prime}$.

We hence get $\mathrm{d}_{\mathcal{X}}\left(t, t^{\prime}\right) \leqslant \ell M$, as required.

Lemma 13.13. Let $\left\{\mathcal{X}_{m}\right\}$ be uniformly hierarchically hyperbolic spaces, with respect to $\left\{\mathfrak{S}_{m}\right\}$, and let $\mathcal{X}$ be an ultralimit of $\left(\mathcal{X}_{m}\right)$. Also, let $\boldsymbol{x}, \boldsymbol{y}$ be distinct points of $\mathcal{X}$. Then there exists an ultralimit $F_{\boldsymbol{U}}$ of the seqeunce $\left(F_{U_{m}}\right)$, an ultralimit $\boldsymbol{\gamma}$ of hierarchy paths $\gamma_{m}$, contained in $F_{\boldsymbol{U}}$ and connecting distinct points $\boldsymbol{x}^{\prime}, \boldsymbol{y}^{\prime}$, and a Lipschitz map $\boldsymbol{q}_{\boldsymbol{\gamma}}: \mathcal{X} \rightarrow \boldsymbol{\gamma}$ such that

(1) $\boldsymbol{q}_{\boldsymbol{\gamma}}(\boldsymbol{x})=\boldsymbol{x}^{\prime}, \boldsymbol{q}_{\boldsymbol{\gamma}}(\boldsymbol{y})=\boldsymbol{y}^{\prime}$

(2) $\boldsymbol{q}_{\boldsymbol{\gamma}}$ restricted to $\boldsymbol{P}_{\boldsymbol{U}}$ is the projection on the first factor, where $\boldsymbol{P}_{\boldsymbol{U}} \cong \boldsymbol{E}_{\boldsymbol{U}} \times \boldsymbol{\gamma}$ is an ultralimit of $\left(P_{U_{m}}\right)$;

(3) $\boldsymbol{q}_{\boldsymbol{\gamma}}$ is locally constant outside $\boldsymbol{P}_{\boldsymbol{U}}$

Proof. We first claim that there exist $U_{m} \in \mathfrak{S}_{m}$ so that

(1) $\lim _{\omega} \frac{1}{s_{m}} \sigma_{U_{m}, s_{0}}\left(x_{m}, y_{m}\right)>0$,

(2) for any $U_{m}^{\prime} \in \mathfrak{S}_{U_{m}}-\left\{U_{m}\right\}$, we have $\lim _{\omega} \frac{1}{s_{m}} \sigma_{U_{m}^{\prime}, s_{0}}\left(x_{m}, y_{m}\right)=0$.

In fact, since $\boldsymbol{x} \neq \boldsymbol{y}$, there exists some $\left(\widehat{\mathcal{C}} U_{m}\right)$ satisfying the first property, by the distance formula and the existence of a $\sqsubseteq$-maximal element. Also, if $\left(\widehat{\mathcal{C}} U_{m}\right)$ satisfies the first property but not the second one, then by definition we have a sequence $\left(\widehat{\mathcal{C}} V_{m}\right)$ satisfying the first property and so that each $V_{m}$ is $\omega$-a.e. properly nested into $U_{m}$. By Definition 13.1. (5), in finitely many steps we find $\left(U_{m}\right)$ with the desired property.

Now let $\gamma_{m}$ be a hierarchy path in $F_{U_{m}}$ connecting $x_{m}^{\prime}=\mathfrak{g}_{P_{U_{m}}}\left(x_{m}\right)$ to $y_{m}^{\prime}=\mathfrak{g}_{P_{U_{m}}}\left(y_{m}\right)$. We can define $\boldsymbol{q}_{\boldsymbol{\gamma}}$ to be the ultralimit of the maps $q_{\gamma_{m}}$ as in Lemma 13.12, and all properties are easily verified.

Proof of Theorem 13.11. The main task is to prove Theorem 13.11. 22). Along the way we will point out how to adapt the first part of the argument to obtain Theorem 13.11.(1).

We will prove the proposition by induction on complexity, the base case being that of complexity 0 , where the distance formula implies that every ultralimit of $\left(\mathcal{X}_{m}\right)$ is a point.

Consider the ultralimit $\mathcal{X}^{1}$ of $\left(\mathcal{X}_{m}\right)$ with scaling factor $\left(r_{m}^{1}\right)$ and an ultralimit map $\boldsymbol{f}^{1}: \boldsymbol{B}^{1} \rightarrow$ $\mathcal{X}^{1}$. (For 13.11, (1), we don't restrict the choice of scaling factors used in forming $\mathcal{X}^{1}$.)

If $\boldsymbol{f}^{1}\left(\boldsymbol{B}^{1}\right)$ is a single point, then the conclusion is immediate. Hence, consider $\boldsymbol{x} \neq \boldsymbol{y}$ in $\boldsymbol{f}^{1}\left(\boldsymbol{B}^{1}\right)$. Let $\boldsymbol{\gamma}, \boldsymbol{U}, \boldsymbol{x}^{\prime}, \boldsymbol{y}^{\prime}$ and $\boldsymbol{q}_{\boldsymbol{\gamma}}: \boldsymbol{X}^{1} \rightarrow \boldsymbol{\gamma}$ be as in Lemma 13.13 . In the situation of 13.11. (1), towards a contradiction, we pick $\boldsymbol{x} \neq \boldsymbol{y}$ in the same ultralimit of cosets of $[\mathcal{N}, \mathcal{N}]$, which we can do if $\mathcal{N}$ is not abelian. It follows by Pansu's Differentiability Theorem [Pan89, Theorem 2], which applies because the asymptotic cones of $\mathcal{N}$ are Carnot groups [Pan83], that every Lipschitz map from $\boldsymbol{B}^{1}$ to $\mathbb{R}$ is constant along cosets of $[\mathcal{N}, \mathcal{N}]$; this contradicts the properties of $\boldsymbol{q}_{\boldsymbol{\gamma}}$ established in Lemma 13.13 when $\mathcal{N}$ is not abelian. Theorem 13.11,(1) is hence proven, and from now on we focus exclusively on Theorem 13.11.(2). By Rademacher's Theorem there exists $\left(p_{m}\right) \in\left(B_{m}^{1}\right)$ so that $\boldsymbol{g}_{\boldsymbol{\gamma}}=\boldsymbol{q}_{\boldsymbol{\gamma}} \circ \boldsymbol{f}^{1}$ is differentiable at $\boldsymbol{p}$ and the differential is nonzero and $\mathrm{d}_{\mathbb{R}^{n}}\left(p_{m}, 0\right) \leqslant r_{m}^{1} / 2$. In particular $B\left(p_{m}, r_{m}^{1} / 2\right) \subseteq B_{m}$. Abusing notation 
slightly, we are regarding $\gamma$ as an interval in $\mathbb{R}$.

Claim 1. For every $\epsilon>0$ there exists $l_{\epsilon}>0$ so that for any $l \leqslant l_{\epsilon}$ we have that $\boldsymbol{f}^{1}(B(\boldsymbol{p}, l))$ is contained in the $\epsilon l$-neighborhood of $\boldsymbol{P}_{\boldsymbol{U}}$.

Proof of Claim 1. We identify a neighborhood of $\boldsymbol{p}$ with a neighborhood of 0 in $\mathbb{R}^{n}$.

We know that there exists a linear function $A$ so that for any $v_{1}$ and $v_{2}$ in this neighborhood we have $\left|\boldsymbol{g}_{\boldsymbol{\gamma}}\left(v_{1}\right)-\boldsymbol{g}_{\boldsymbol{\gamma}}\left(v_{2}\right)\right|=\left|A\left(v_{2}-v_{1}\right)\right|+o\left(\max \left\{\left\|v_{1}\right\|,\left\|v_{2}\right\|\right\}\right)$. Moreover, consider $v$ of norm 1 and set $A v=\theta>0$. Given any $v_{1}$ with $d\left(\boldsymbol{f}^{1}\left(v_{1}\right), \boldsymbol{E}_{\boldsymbol{U}} \times \gamma\right) \geqslant \epsilon\left\|v_{1}\right\|$, then, since $\boldsymbol{f}^{1}$ is $K-$ Lipschitz and $\boldsymbol{q}_{\boldsymbol{\gamma}}$ is constant outside $\boldsymbol{E}_{\boldsymbol{U}} \times \boldsymbol{\gamma}$, we have $\boldsymbol{g}_{\boldsymbol{\gamma}}\left(v_{1}\right)=\boldsymbol{g}_{\boldsymbol{\gamma}}\left(v_{2}\right)$, where $v_{2}=v_{1}+\frac{\epsilon\left\|v_{1}\right\|}{K} v$. Hence

$$
0=\left|\boldsymbol{g}_{\boldsymbol{\gamma}}\left(v_{1}\right)-\boldsymbol{g}_{\boldsymbol{\gamma}}\left(v_{2}\right)\right|=\frac{\epsilon \theta\left\|v_{1}\right\|}{K}+o\left((1+\epsilon / K)\left\|v_{1}\right\|\right)
$$

which cannot happen for $\left\|v_{1}\right\|$ small enough depending on $\epsilon$.

Claim 2. There exists $\left(r_{m}^{2}\right)$ with $\left(r_{m}^{0}\right) \leqslant\left(r_{m}^{2}\right) \ll\left(r_{m}^{1}\right)$ so that for all $\left(r_{m}\right)$ with $\left(r_{m}^{2}\right) \ll$ $\left(r_{m}\right) \ll\left(r_{m}^{1}\right)$ we have $\boldsymbol{f}(\boldsymbol{B}) \subseteq \boldsymbol{E}_{\boldsymbol{U}^{\prime}} \times \gamma^{\prime}$, where $\boldsymbol{B}$ is the ultralimit of $\left(B_{m}\right)$ with observation point $\left(p_{m}\right)$ and scaling factor $\left(r_{m}\right)$.

Claim 2 follows from Claim 1 by an application of the principle from nonstandard analysis called underspill, nonetheless, we provide a proof in the interest of self-containment.

Proof of Claim 2. In view of Claim 1, there exists a function $\epsilon: \mathbb{N} \rightarrow \mathbb{R}^{+}$so that $\epsilon(k) \rightarrow 0$ as $k \rightarrow \infty$ and so that for every $k$ the set $A_{k} \subseteq \mathbb{N}$ defined below satisfies $\omega\left(A_{k}\right)=1$,

$$
A_{k}=\left\{m \mid f_{m}\left(B\left(p_{m}, r_{m}^{1} / 2^{k}\right)\right) \subseteq \mathcal{N}_{\epsilon(k) r_{m}^{1} / 2^{k}}\left(E_{U_{m}} \times \gamma_{m}\right)\right\} .
$$

Let $k(m)=\max \left\{k \leqslant m: m \in \bigcap_{i \leqslant k} A_{i}\right\}$, which by the above is well-defined $\omega$-a.e. and satisfies $\lim _{\omega} k(m)=\infty$. By definition of $k(m)$, for $\omega$-a.e. $m$ we have $f_{m}\left(B\left(p_{m}, r_{m}^{1} / 2^{k}\right)\right) \subseteq$ $\mathcal{N}_{\epsilon(k) r_{m}^{1} / 2^{k}}\left(E_{U_{m}} \times \gamma_{m}\right)$ for every $k \leqslant k(m)$.

Let $\left(r_{m}^{2}\right)=\max \left\{\left(r_{m}^{0}\right),\left(r_{m}^{1} / 2^{k(m)}\right)\right\}$. We claim that if $\left(r_{m}^{2}\right) \ll\left(r_{m}\right) \ll\left(r_{m}^{1}\right)$, then the result holds for the scaling factor $\left(r_{m}\right)$. To do this, we show for each $j \in \mathbb{N}$ that $\omega\left(A_{j}^{\prime}\right)=1$, where:

$$
A_{j}^{\prime}=\left\{m \mid f_{m}\left(B\left(p_{m}, j r_{m}\right)\right) \subseteq \mathcal{N}_{r_{m} / j}\left(E_{U_{m}} \times \gamma_{m}\right)\right\} .
$$

Fix $j$. For a given $m$, let $k^{\prime}(m)$ satisfy $r_{m}^{1} / 2^{k^{\prime}(m)+1}<j r_{m} \leqslant r_{m}^{1} / 2^{k^{\prime}(m)}$. For $\omega$-a.e. $m$ we have $k^{\prime}(m) \leqslant k(m)$ since $\left(r_{m}\right) \gg\left(r_{m}^{1} / 2^{k(m)}\right)$. In particular for $\omega$-a.e. $m$ we have:

$f_{m}\left(B\left(p_{m}, j r_{m}\right)\right) \subseteq f_{m}\left(B\left(p_{m}, r_{m}^{1} / 2^{k^{\prime}(m)}\right)\right) \subseteq \mathcal{N}_{\epsilon\left(k^{\prime}(m)\right) r_{m}^{1} / 2^{k^{\prime}(m)}}\left(E_{U_{m}} \times \gamma_{m}\right) \subseteq \mathcal{N}_{2 \epsilon\left(k^{\prime}(m)\right) j r_{m}}\left(E_{U_{m}} \times \gamma_{m}\right)$.

Since $\left(r_{m}\right) \ll\left(r_{m}^{1}\right)$, we have $\lim _{\omega} k^{\prime}(m)=\infty$, and hence $2 \epsilon\left(k^{\prime}(m)\right) \leqslant 1 / j^{2}$ for $\omega$-a.e. $m$; the claim follows.

By induction on complexity, enabled by Remark 13.6, we know that for some $\left(r_{m}\right) \gg\left(r_{m}^{2}\right)$ the ultralimit $\boldsymbol{g}$ of $g_{m}=\mathfrak{g}_{E_{U_{m}}} \circ f_{m}$ maps $\boldsymbol{B}$ into some ultralimit $\boldsymbol{F}^{\prime}$ of standard boxes, whence it is readily deduced that $\boldsymbol{f}(\boldsymbol{B})$ is contained in the ultralimit $\boldsymbol{F}^{\prime} \times \boldsymbol{\gamma}^{\prime}$ of standard boxes. Note that provided $r_{m} \leqslant r_{m}^{1} / 2$, the ultralimit $\mathbf{B}$ contains the required ultralimit of $\left(B\left(p_{m}, r_{m}\right)\right)$.

\section{ACYLINDRICITY}

Definition 14.1 (Automorphism of a hierarchically hyperbolic space). Let $\mathcal{X}$ be a hierarchically hyperbolic space, with respect to $\mathfrak{S}$. An automorphism $g$ of $(\mathcal{X}, \mathfrak{S})$ is a bijection $g: \mathfrak{S} \rightarrow \mathfrak{S}$ and a collection $\left\{g_{U}: \widehat{\mathcal{C}} U \rightarrow \widehat{\mathcal{C}}(g U): U \in \mathfrak{S}\right\}$ of isometries such that for all $U, V \in \mathfrak{S}$ we have $g_{V}\left(\rho_{V}^{U}\right)=\rho_{g V}^{g U}$ and such that $g$ preserves $\perp, \sqsubseteq$, and $\pitchfork$. 
By Definition 13.1. (6), (9), every automorphism $g$ of $\mathfrak{S}$ induces a quasi-isometry $\phi_{g}: \mathcal{X} \rightarrow$ $\mathcal{X}$ with uniformly bounded constants. Such a quasi-isometry $\phi_{g}$ can be described as follows. For $x \in \mathcal{X}$ and $U \in \mathfrak{S}, \pi_{g U}\left(\phi_{g}(x)\right)$ coarsely coincides with $g_{U}\left(\pi_{U}(x)\right)$. When it will not introduce confusion, we will use the notation " $g$ " for the quasi-isometry $\phi_{g}$ as well as for the element $g \in \operatorname{Aut}(\mathfrak{S})$. In the remainder of this section, we are interested in the situation where the action of $G$ on $\mathcal{X}$ by uniform quasi-isometries is proper and cocompact.

Let $S=S(\mathcal{X}, \mathfrak{S})$ be the unique $\sqsubseteq$-maximal element and consider $G \leqslant \operatorname{Aut}(\mathfrak{S})$. Since each $g \in G$ preserves the nesting relation, $G$ fixes $S$, and hence $g_{S}$ is an isometry of $\hat{\mathcal{C}} S$ for each $g \in G$. Note that this action coarsely preserves the union of the sets in $\pi_{S}(\mathcal{X})$.

Remark 14.2. When $\mathcal{X}$ is a cube complex with a factor system, $S=\mathcal{X}$ and $\widehat{\mathcal{C}} S$ is the factored contact graph $\widehat{\mathcal{C}} \mathcal{X}$. The union of sets in the image of $\pi_{\mathcal{X}}: \mathcal{X} \rightarrow 2^{\hat{\mathcal{C}} \mathcal{X}^{(0)}}$ is coarsely equal to $\widehat{\mathcal{C}} \mathcal{X}$, by the definition of $\pi_{\mathcal{X}}$.

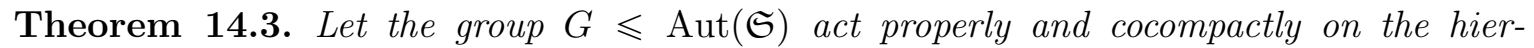
archically hyperbolic space $\mathcal{X}$ and let $S$ be the unique $\sqsubseteq$-maximal element of $\mathfrak{S}$. Then for all $\epsilon>0$, there exist constants $R, N$ such that there are at most $N$ elements $g \in$ $G$ such that $\mathrm{d}_{\widehat{\mathcal{C}} S}\left(\pi_{S}(x), \pi_{S}(g x)\right)<\epsilon, \mathrm{d}_{\hat{\mathcal{C}} S}\left(\pi_{S}(y), \pi_{S}(g y)\right)<\epsilon$ whenever $x, y \in \mathcal{X}$ satisfy $\mathrm{d}_{\widehat{\mathcal{C}} S}\left(\pi_{S}(x), \pi_{S}(y)\right) \geqslant R$.

Before proving Theorem 14.3, we note two corollaries. Recall that the action of the group $G$ on the metric space ( $M, \mathrm{~d})$ is acylindrical if for all $\epsilon>0$, there exist constants $R, N$ such that there are at most $N$ elements $g \in G$ such that $\mathrm{d}(x, g x)<\epsilon, \mathrm{d}(y, g y)<\epsilon$ whenever $x, y \in M$ satisfy $\mathrm{d}(x, y) \geqslant R$. (Note that if $M$ is bounded, then any action on $M$ is automatically acylindrical. In particular, if $\mathcal{X}$ is a cube complex decomposing as the product of unbounded subcomplexes and $G$ acts geometrically on $\mathcal{X}$, then the action of $G$ on $\mathcal{C} \mathcal{X}$ is trivially acylindrical.)

Corollary 14.4 (Acylindrical hyperbolicity). Let $G \leqslant \operatorname{Aut}(\mathfrak{S})$ act properly and cocompactly on the hierarchically hyperbolic space $\mathcal{X}$. Then $G$ acts acylindrically on a hyperbolic space quasi-isometric to $\mathcal{U}=\bigcup_{x \in \mathcal{X}} \pi_{S}(x)$. In particular, if $\mathcal{U}$ is unbounded and $G$ is not virtually cyclic, then $G$ is acylindrically hyperbolic.

Proof. Let $S$ be as in Theorem 14.3. Let $\mathcal{T}_{0} \subseteq \widehat{\mathcal{C}} S$ be the union of all sets of the form $\pi_{S}(x)$ with $x \in \mathcal{X}$, together with all of their $G$-orbits, so that $\mathcal{T}_{0}$ is $G$-invariant and coarsely equal to the union of the elements of $\pi_{S}(\mathcal{X})$. Definition 13.1.,10) thus ensures that $\mathcal{T}_{0}$ is quasiconvex in the hyperbolic space $\widehat{\mathcal{C}} S$, so that we can add geodesics of $\widehat{\mathcal{C}} S$ to $\mathcal{T}_{0}$ to form a $G$-hyperbolic space $\mathcal{T}$ that is $G$-equivariantly quasi-isometric to the union of the elements of $\pi_{S}(\mathcal{X})$. The action of $G$ on $\mathcal{T}$ is thus acylindrical by Theorem 14.3 .

In the cubical case, acylindricity can be witnessed by the contact graph instead of the factored contact graph.

Corollary 14.5. Let $G$ act properly and cocompactly on the $C A T(0)$ cube complex $\mathcal{X}$, and suppose that $\mathcal{X}$ contains a $G$-invariant factor system. Then $G$ acts acylindrically on $\hat{\mathcal{C}} \mathcal{X}$, and hence on $\mathcal{C X}$.

Proof. For any $G$-invariant factor system $\mathfrak{F}$, the action of $G$ on $\hat{\mathcal{C}} \mathcal{X}$ is acylindrical by Theorem 14.3 and Remarks 13.2 and 14.2 . Let $\mathfrak{F}$ be a factor-system in $\mathcal{X}$ and let $\xi$ be the constant for $\mathfrak{F}$ from Definition 8.1. Let $\mathfrak{F}_{0}$ be the smallest set of convex subcomplexes of $\mathcal{X}$ that contains $\mathcal{X}$ and each subcomplex parallel to a combinatorial hyperplane, and has the property that $\mathfrak{g}_{F}\left(F^{\prime}\right) \in \mathfrak{F}_{0}$ whenever $F, F^{\prime} \in \mathfrak{F}_{0}$ and $\operatorname{diam}\left(\mathfrak{g}_{F}\left(F^{\prime}\right)\right) \geqslant \xi$. By definition, $\mathfrak{F}_{0} \subseteq \mathfrak{F}$, so $\mathfrak{F}_{0}$ has bounded multiplicity and thus $\mathfrak{F}_{0}$ is a factor system. The associated factored contact 
graph of $\mathcal{X}$, on which $G$ acts acylindrically, is $G$-equivariantly quasi-isometric to $\mathcal{C} \mathcal{X}$, and the result follows.

Proof of Theorem 14.3. Fix $\epsilon>0$, and for convenience assume that it is 100 times larger than all the constants in Definition 13.1. Let $R_{0} \geqslant 1000 \epsilon$ and consider $x, y \in \mathcal{X}$ such that $R=\mathrm{d}_{\widehat{\mathcal{C}} S}\left(\pi_{S}(x), \pi_{S}(y)\right) \geqslant R_{0}$, and let $\mathfrak{H}$ be the set of all $g \in G$ such that $\mathrm{d}_{\widehat{\mathcal{C}} S}\left(\pi_{S}(g x), \pi_{S}(x)\right)<$ $\epsilon$ and $\mathrm{d}_{\widehat{\mathcal{C}} S}\left(\pi_{S}(g y), \pi_{S}(y)\right)<\epsilon$.

We will consider, roughly speaking, the set of all $U \in \mathfrak{S}$ so that $x, y$ project far away in $U$, the corresponding $\rho_{S}^{U}$ is near the middle of a geodesic from $\pi_{S}(x)$ to $\pi_{S}(y)$, and $U$ is $\sqsubseteq$-maximal with these properties. We do so because these $U$ correspond to product regions "in the middle" between $x$ and $y$. Formally, let $\mathfrak{L}_{1}$ be the set of all $U \in \mathfrak{S}-\{S\}$ with the following properties:

(1) $\mathrm{d}_{\widehat{\mathcal{C}} U}\left(\pi_{U}(x), \pi_{U}(y)\right)>\epsilon$;

(2) $\left|\mathrm{d}_{\widehat{\mathcal{C}} S}\left(\pi_{S}(x), \rho_{S}^{U}\right)-\frac{R}{2}\right| \leqslant 10 \epsilon$

(3) $U$ is not properly nested into any $U^{\prime} \in \mathfrak{S}-\{S\}$ with $\mathrm{d}_{\widehat{\mathcal{C}} U^{\prime}}\left(\pi_{U^{\prime}}(x), \pi_{U^{\prime}}(y)\right)>\epsilon$.

When applying an element $g$ that moves $x, y$ a bounded amount, any $U \in \mathfrak{L}_{1}$ gets moved to some $g U$ with similar properties but slightly worse constants. To capture this, we let $\mathfrak{L}_{2}$ be the set of all $U \in \mathfrak{S}-\{S\}$ such that:

(1) $\mathrm{d}_{\widehat{\mathcal{C}} U}\left(\pi_{U}(x), \pi_{U}(y)\right)>\epsilon / 2$

(2) $\left|\mathrm{d}_{\hat{\mathcal{C}} S}\left(\pi_{S}(x), \rho_{S}^{U}\right)-\frac{R}{2}\right| \leqslant 11 \epsilon$

(3) $U$ is not properly nested into any $U^{\prime} \in \mathfrak{S}-\{S\}$ with $\mathrm{d}_{\widehat{\mathcal{C}} U^{\prime}}\left(\pi_{U^{\prime}}(x), \pi_{U^{\prime}}(y)\right)>2 \epsilon$.

Bounding $\left|\mathfrak{L}_{2}\right|$ : Consider a hierarchy path $\gamma$ from $x$ to $y$. Then there are $x^{\prime}, y^{\prime}$ on $\gamma$ so that

- $\mathrm{d}_{\widehat{\mathcal{C}} S}\left(\pi_{S}\left(x^{\prime}\right), \pi_{S}\left(y^{\prime}\right)\right) \leqslant 23 \epsilon$, and

- whenever $U \in \mathfrak{S}-\{S\}$ is so that $\mathrm{d}_{\widehat{\mathcal{C}} U}\left(\pi_{U}(x), \pi_{U}(y)\right)>\epsilon / 2$ and $\left|\mathrm{d}_{\widehat{\mathcal{C}} S}\left(\pi_{S}(x), \rho_{S}^{U}\right)-\frac{R}{2}\right| \leqslant$ $11 \epsilon$, then $\mathrm{d}_{\hat{\mathcal{C}} U}\left(\pi_{U}\left(x^{\prime}\right), \pi_{U}\left(y^{\prime}\right)\right)>s_{0}$ (recall that $s_{0}$ is the minimal threshold of the Distance Formula).

The existence of $x^{\prime}, y^{\prime}$ follows since we can choose $x^{\prime}$ and $y^{\prime}$ projecting close to points on a $\widehat{\mathcal{C}} S$-geodesic from $\pi_{S}(x)$ to $\pi_{S}(y)$ that lie on opposite sides of the midpoint, at distance slightly larger than $11 \epsilon$ from the midpoint. Bounded Geodesic Image (Definition 13.1. (8)) guarantees that the second condition holds because it ensures that $\pi_{U}(x), \pi_{U}(y)$ coarsely coincide with $\pi_{U}\left(x^{\prime}\right), \pi_{U}\left(y^{\prime}\right)$ (recall that $\epsilon$ is much larger than $s_{0}$ and all other constants in Definition 13.1.

By Definition 13.1. (7) (Large Link Lemma), with $W=S$ and $x, x^{\prime}$ replaced by $x^{\prime}, y^{\prime}$, each $U \in \mathfrak{L}_{2}$ is nested into one of at most $23 \epsilon \lambda+\lambda$ elements $T$ of $\mathfrak{S}$. For $p$ as in the Claim below, the number of $U \in \mathfrak{L}_{2}$ nested into the same such $T$ is bounded by $p$, for otherwise some $U$ (the $U_{i}$ in the conclusion of the Claim) would fail to satisfy the third property in the definition of $\mathfrak{L}_{2}$. Hence $\left|\mathfrak{L}_{2}\right| \leqslant p \lambda(23 \epsilon+1)$.

Claim. There exists $p$ with the following property. Let $T \in \mathfrak{S}$, let $x, y \in \mathcal{X}$, and let $\left\{U_{i}\right\}_{i=1}^{p} \subseteq \mathfrak{S}_{T}$ be distinct and satisfy $\mathrm{d}_{\widehat{\mathcal{C}} U_{i}}\left(\pi_{U_{i}}(x), \pi_{U_{i}}(y)\right) \geqslant \epsilon$. Then there exists $U^{\prime} \in \mathfrak{S}_{T}$ and $i$ so that $U_{i} \sqsubseteq U^{\prime}$ and $\mathrm{d}_{\widehat{\mathcal{C}} U^{\prime}}\left(\pi_{U^{\prime}}(x), \pi_{U^{\prime}}(y)\right)>2 \epsilon$.

Proof of Claim. Define the level of $Y \in \mathfrak{S}$ to be the maximal $k$ so that there exists a $\sqsubseteq$-chain of length $k$ in $\mathfrak{S}_{Y}$. The proof is by induction on the level $k$ of a $\sqsubseteq-$ minimal $T^{\prime} \in \mathfrak{S}_{T}$ into which each $S_{i}$ is nested. For the base case $k=1$ it suffices to take $p=2$ since in this case there is no pair of distinct $U_{1}, U_{2} \in \mathfrak{S}_{T^{\prime}}$.

Suppose that the statement holds for a given $p(k)$ when the level of $T^{\prime}$ is at most $k$. Suppose further that $\left|\left\{U_{i}\right\}\right| \geqslant p(k+1)$ (where $p(k+1)$ is a constant much larger than $p(k)$ 
that will be determined shortly) and there exists a $\sqsubseteq$-minimal $T^{\prime} \in \mathfrak{S}_{T}$ of level $k+1$ into which each $U_{i}$ is nested. There are two cases.

If $\mathrm{d}_{\hat{\mathcal{C}} T^{\prime}}\left(\pi_{T^{\prime}}(x), \pi_{T^{\prime}}(y)\right)>2 \epsilon$, then we are done (for $p \geqslant 2$ ). If not, then Definition 13.1. (7) (Large Link Lemma) yields $K$ and $T_{1}, \ldots, T_{K}$, each properly nested into $T^{\prime}$ (and hence of level $\leqslant k)$, so that any given $U_{i}$ is nested into some $T_{j}$. In particular, if $p(k+1) \geqslant K p(k)$, there exists $j$ so that at least $p(k)$ elements of $\left\{U_{i}\right\}$ are nested into $T_{j}$. By the induction hypothesis and Definition 13.1.5. (Finite Complexity), we are done.

$\mathfrak{L}_{1} \neq \varnothing$ case: Suppose that there exists $U \in \mathfrak{L}_{1}$. In this case the idea is that there actually are product regions in the middle between $x, y$, and the action of an element moving $x, y$ not too much permutes the gates into such product regions, so that there cannot be too many such elements.

First of all, our choice of $R_{0}$ and Definition 13.1.(8) (Bounded Geodesic Image) ensure that for all $g \in \mathfrak{H}$, we have $g U \in \mathfrak{L}_{2}$. Indeed, if $U \in \mathfrak{L}_{1}$ then $\mathrm{d}_{\widehat{\mathcal{C}} g U}\left(\pi_{g U}(x), \pi_{g U}(y)\right) \geqslant$ $\mathrm{d}_{\hat{\mathcal{C}} g U}\left(\pi_{g U}(g x), \pi_{g U}(g y)\right)-2 B \geqslant \epsilon-2 B>\epsilon / 2$, ensuring the first property in the definition of $\mathfrak{L}_{2}$. The second property follows from the fact that $\pi_{S}(x)$ gets moved distance $\leqslant \epsilon$ by $g$, and the third property holds for $g U$ because otherwise, using Bounded Geodesic Image, we would find a contradiction with the third property of $U$ from the definition of $\mathfrak{L}_{1}$.

Fix any $g \in \mathfrak{H}$ and let $P_{1}=E_{U} \times F_{U}, P_{2}=E_{g U} \times F_{g U}$ be the spaces provided by Section 13.1 . We claim that $\mathrm{d}_{\mathcal{X}}\left(g \mathfrak{g}_{P_{1}}(x), \mathfrak{g}_{P_{2}}(x)\right)$ is uniformly bounded. From this, properness of the action and the bound on $\left|\mathfrak{L}_{2}\right|$ yield a bound on $|\mathfrak{H}|$.

Clearly, $g \mathfrak{g}_{P_{1}}(x)$ coarsely coincides with $\mathfrak{g}_{P_{2}}(g x)$, since coordinates of gates are defined equivariantly. Hence we must show that $w=\mathfrak{g}_{P_{2}}(g x)$ coarsely coincides with $z=\mathfrak{g}_{P_{2}}(x)$.

By Definition 13.1.(6) (Distance Formula), it suffices to show that the projections of $w$ and $z$ coarsely coincide in every $\widehat{\mathcal{C}} Y$ for $Y \in \mathfrak{S}$. By definition of $\mathfrak{g}_{P_{2}}$, it suffices to consider $Y \in \mathfrak{S}$ which is either nested into or orthogonal to $g U$. For such $Y, \rho_{S}^{Y}$ coarsely coincides with $\rho_{S}^{g U}$ by the final part of Definition 13.1.(4) (Transversality and consistency). Moreover, any geodesic from $x$ to $g x$ stays far from $\rho_{S}^{Y}$, so that Definition 13.1. (8) (Bounded Geodesic Image) gives a uniform bound on $\mathrm{d}_{\widehat{\mathcal{C}} Y}\left(\pi_{Y}(x), \pi_{Y}(g x)\right)$, as required.

$\mathfrak{L}_{1}=\varnothing$ case: Suppose now that $\mathfrak{L}_{1}=\varnothing$. In this case, the idea is that a hierarchy path from $x$ to $y$ does not spend much time in any product region near the middle, and hence it behaves like a geodesic in a hyperbolic space. Fix a hierarchy path $\gamma$ in $\mathcal{X}$ joining $x, y$ and let $p \in \gamma$ satisfy $\left|\mathrm{d}_{\widehat{\mathcal{C}} S}\left(\pi_{S}(p), \pi_{S}(x)\right)-\frac{R}{2}\right| \leqslant \epsilon$. We will produce a constant $M_{3}$, depending on the constants from Definition 13.1 and on $\epsilon$ such that $\mathrm{d}_{\mathcal{X}}(p, g p) \leqslant M_{3}$. It will then follow that $|\mathfrak{H}|$ is bounded in view of properness of the action of $G$ on $\mathcal{X}$.

We now bound $\mathrm{d}_{\mathcal{X}}(p, g p)$ using the distance formula. First, note that $\mathrm{d}_{\widehat{\mathcal{C}} S}\left(\pi_{S}(p), \pi_{S}(g p)\right) \leqslant$ $10 \delta+\epsilon$ (where $\delta$ is the hyperbolicity constant of $\widehat{\mathcal{C}} S$ ).

If $U \in \mathfrak{S}$ contributes to the sum $\sigma_{\mathcal{X}, s_{0}}(p, g p)$ with threshold $s_{0}$, then, given the bound on $\mathrm{d}_{\widehat{\mathcal{C}} S}(p, g p)$ and Bounded Geodesic Image, $\mathrm{d}_{\widehat{\mathcal{C}} S}\left(\pi_{S}(p), \rho_{S}^{U}\right) \leqslant 2 \epsilon$. Fix now any $U$ satisfying $\mathrm{d}_{\widehat{\mathcal{C}} S}\left(\pi_{S}(p), \rho_{S}^{U}\right) \leqslant 2 \epsilon$. Our goal is now to bound $\mathrm{d}_{\widehat{\mathcal{C}} U}\left(\pi_{U}(p), \pi_{U}(g p)\right)$ uniformly. It follows from the assumption that $\mathfrak{L}_{1}=\varnothing$ that $d_{\widehat{\mathcal{C}} U}\left(\pi_{U}(x), \pi_{U}(y)\right) \leqslant 3 \epsilon$, and also $d_{\widehat{\mathcal{C}} U}\left(\pi_{U}(g x), \pi_{U}(g y)\right)=$ $d_{\widehat{\mathcal{C}}_{g^{-1} U}}\left(\pi_{g^{-1} U}(x), \pi_{g^{-1} U}(y)\right) \leqslant 3 \epsilon$. Indeed, if, say, $U$ satisfied $d_{\widehat{\mathcal{C}} U}\left(\pi_{U}(x), \pi_{U}(y)\right)>3 \epsilon$, then either $U$ or some other $U^{\prime}$ with $d_{\widehat{\mathcal{C}} U^{\prime}}\left(\pi_{U^{\prime}}(x), \pi_{U^{\prime}}(y)\right)>\epsilon$ and $U \sqsubseteq U^{\prime}$ would belong to $\mathfrak{L}_{1}$, since for $U \sqsubseteq U^{\prime}$ and $U^{\prime} \neq S$, the sets $\rho_{S}^{U}$ and $\rho_{S}^{U^{\prime}}$ coarsely coincide.

Since $\gamma$ is a hierarchy path, $\mathrm{d}_{\widehat{\mathcal{C}} U}\left(\pi_{U}(x), \pi_{U}(p)\right)$ and $d_{\hat{\mathcal{C}} U}\left(\pi_{U}(g x), \pi_{U}(g p)\right)$ are bounded by $\mathrm{d}_{\hat{\mathcal{C}} U}\left(\pi_{U}(x), \pi_{U}(y)\right)+2 D$ and $d_{\hat{\mathcal{C}} U}\left(\pi_{U}(g x), \pi_{U}(g y)\right)+2 D$, respectively, which are both bounded by, say, $4 \epsilon$. Hence, $\mathrm{d}_{\widehat{\mathcal{C}} U}\left(\pi_{U}(p), \pi_{U}(g p)\right) \leqslant 9 \epsilon+\mathrm{d}_{\widehat{\mathcal{C}} U}\left(\pi_{U}(x), \pi_{U}(g x)\right)$ (where the diameters of 
the projection sets are taken care of by the extra $\epsilon$ ), and the last term is uniformly bounded by Bounded Geodesic Image. We then get the desired bound, concluding the proof.

Corollary 14.6. Let $G \leqslant \operatorname{Aut}(\mathfrak{S})$ act properly and cocompactly on the hierarchically hyperbolic space $\mathcal{X}$. Let $g, h \in G$ be hyperbolic on the maximal $S \in \mathfrak{S}$ and satisfy $g^{n} h \neq h g^{n}, h^{n} g \neq$ $g h^{n}$ for all $n \neq 0$. Then there exists $N>0$, depending on $g$ and $h$, such that $\left\langle g^{N}, h^{N}\right\rangle$ is freely generated by $g^{N}, h^{N}$.

Proof. By Corollary 14.4 $G$ acts acylindrically on a hyperbolic space, whence the claim follows from [Fuj08, Proposition 2.4].

In particular, when $G$ acts properly and cocompactly on a CAT(0) cube complex with a factor system, e.g., when $G$ is compact special, the conclusion of Corollary 14.6 is satisfied. Thus acylindricity can be used to find free subgroups of groups acting on cube complexes by different means than are used in the discussion of the Tits alternative [CS11, SW05. The above corollary recovers Theorem 47 of [KK14] about subgroups of right-angled Artin groups generated by powers of elements acting loxodromically on the extension graph once we observe, as in [KK14], that there is a quasi-isometry from the extension graph to the contact graph (whenever the right-angled Artin group does not have a free $\mathbb{Z}$ factor).

In the case $\mathcal{X}$ is a uniformly locally finite cube complex, not necessarily equiped with a factor system, one can obtain the same conclusion, provided $g, h \in \operatorname{Aut}(\mathcal{X})$ act loxodromically on $\mathcal{C} \mathcal{X}$, by a ping-pong argument.

\section{REFERENCES}

[Ago13] Ian Agol. The virtual Haken conjecture (with an appendix by Ian Agol, Daniel Groves and Jason Manning). Doc. Math., J. DMV, 18:1045-1087, 2013.

[BBFa] M. Bestvina, K. Bromberg, and K. Fujiwara. Bounded cohomology via quasi-trees. arXiv:1306.1542.

[BBFb] Mladen Bestvina, Kenneth Bromberg, and Koji Fujiwara. Constructing group actions on quasitrees and applications to mapping class groups. arXiv:1006.1939.

[BC08] H.-J. Bandelt and V. Chepoi. Metric graph theory and geometry: a survey. In J. E. Goodman, J. Pach, and R. Pollack, editors, Surveys on Discrete and Computational Geometry: Twenty Years Later, volume 453, pages 49-86. Contemp. Math., AMS, Providence, RI, 2008.

[BC12] J. Behrstock and R. Charney. Divergence and quasimorphisms of right-angled Artin groups. Math. Ann., 352:339-356, 2012.

[BCM12] Jeffrey F. Brock, Richard D. Canary, and Yair N. Minsky. The classification of Kleinian surface groups, II: The ending lamination conjecture. Ann. of Math. (2), 176(1):1-149, 2012.

[Beh06] J. Behrstock. Asymptotic geometry of the mapping class group and Teichmüller space. Geometry E Topology, 10:2001-2056, 2006.

[BF] M. Bestvina and M. Feighn. Subfactor projections. J. of Topology. To appear.

[BF02] M. Bestvina and K. Fujiwara. Bounded cohomology of subgroups of mapping class groups. Geometry \& Topology, 6:69-89 (electronic), 2002.

[BH16] J. Behrstock and M.F. Hagen. Cubulated groups: thickness, relative hyperbolicity, and simplicial boundaries. Geometry, Groups, and Dynamics, 10(2):649-707, 2016. ARXIV:1212.0182.

[BHS15] J. Behrstock, M.F. Hagen, and A. Sisto. Hierarchically hyperbolic spaces II: combination theorems and the distance formula. ARXIV:1509.00632, 2015.

[BKMM12] Jason Behrstock, Bruce Kleiner, Yair Minsky, and Lee Mosher. Geometry and rigidity of mapping class groups. Geom. Topol, 16(2):781-888, 2012.

[BKS] M. Bestvina, B. Kleiner, and M. Sageev. Quasiflats in $C A T(0)$ complexes. arXiv:0804.2619.

[BM00] Marc Burger and Shahar Mozes. Lattices in product of trees. Inst. Hautes Études Sci. Publ. Math., 92(1):151-194, 2000.

[BM02] Marc Burger and Nicolas Monod. Continuous bounded cohomology and applications to rigidity theory. Geometric and Functional Analysis, 12(2):219-280, 2002.

[BM08] Jason A Behrstock and Yair N Minsky. Dimension and rank for mapping class groups. Annals of Mathematics, 167:1055-1077, 2008. 
[Bow08] Brian H Bowditch. Tight geodesics in the curve complex. Inventiones mathematicae, 171(2):281300, 2008.

[Bro03] Jeffrey Brock. The weil-petersson metric and volumes of 3-dimensional hyperbolic convex cores. Journal of the American Mathematical Society, 16(3):495-535, 2003.

[BW13] Nicolas Bergeron and Daniel T. Wise. A boundary criterion for cubulation. Amer. J. Math., 2013.

[CD95] Ruth Charney and Michael W. Davis. Finite $K(\pi, 1)$ s for Artin groups. In Prospects in topology (Princeton, NJ, 1994), volume 138 of Ann. of Math. Stud., pages 110-124. Princeton Univ. Press, Princeton, NJ, 1995.

[CH13] Victor Chepoi and Mark F. Hagen. On embeddings of CAT(0) cube complexes into products of trees via colouring their hyperplanes. J. Combin. Theor., Ser. B, 103(4):428 - 467, 2013.

[Cha07] Ruth Charney. An introduction to right-angled artin groups. Geometriae Dedicata, 125:141-158, 2007.

[Che00] Victor Chepoi. Graphs of some CAT(0) complexes. Adv. in Appl. Math., 24(2):125-179, 2000.

[CM09] Pierre-Emmanuel Caprace and Nicolas Monod. Isometry groups of non-positively curved spaces: structure theory. Journal of Topology, pages 661Đ-700, 2009.

[CN05] Indira Chatterji and Graham Niblo. From wall spaces to CAT(0) cube complexes. Internat. J. Algebra Comput., 15(5-6):875-885, 2005.

[CS11] Pierre-Emmanuel Caprace and Michah Sageev. Rank rigidity for CAT(0) cube complexes. Geom. Funct. Anal., 21:851-891, 2011.

[Dav08] Michael W. Davis. The geometry and topology of Coxeter groups, vol. 32 of London Mathematical Society Monographs Series. Princeton University Press, 2008.

[Dru02] Cornelia Druţu. Quasi-isometry invariants and asymptotic cones. International Journal of Algebra and Computation, 12(01n02):99-135, 2002.

[Dur] Matthew Durham. The augmented marking complex of a surface. arXiv:1309.4065.

[EMR] Alex Eskin, Howard Masur, and Kasra Rafi. Large scale rank of Teichmuller space. arXiv:1307.3733.

[Fuj08] Koji Fujiwara. Subgroups generated by two pseudo-anosov elements in a mapping class group. i. uniform exponential growth. Advanced Studies in Pure Mathematics, 20, 2008.

[Hag08] Frédéric Haglund. Finite index subgroups of graph products. Geometriae Dedicata, 135(1):167209, 2008.

[Hag13] Mark F. Hagen. The simplicial boundary of a CAT(0) cube complex. Alg. Geom. Topol., 13:12991367, 2013.

[Hag14] Mark F. Hagen. Weak hyperbolicity of cube complexes and quasi-arboreal groups. J. Topol., $7(2): 385-418,2014$.

[Ham] U. Hamenstädt. Geometry of the mapping class groups III: Quasi-isometric rigidity. arXiv:math.GT/0512429.

[HP13] Mark F. Hagen and Piotr Przytycki. Cocompactly cubulated graph manifolds. To appear in Isr. J. Math. arXiv:1310.1309, 2013.

[Hua] Jingyin Huang. Top dimensional quasiflats in CAT(0) cube complexes. arXiv:1410.8195.

[Hum] David Hume. Embedding mapping class groups into finite products of trees. arXiv:120\%.2132.

[HW08] Frédéric Haglund and Daniel T. Wise. Special cube complexes. Geom. Funct. Anal., 17(5):1 551-1620, 2008.

[HW14] Mark F. Hagen and Daniel T. Wise. Cubulating hyperbolic free-by-cyclic groups: the general case. To appear in GAFA, 2014.

[Iva97] Nikolai V. Ivanov. Automorphism of complexes of curves and of Teichmüller spaces. International Mathematics Research Notices, 1997(14):651-666, 1997.

[KK13] Sang-hyun Kim and Thomas Koberda. Embedability between right-angled Artin groups. Geometry \& Topology, 17:493-530, 2013.

[KK14] Sang-Hyun Kim and Thomas Koberda. The geometry of the curve graph of a right-angled Artin group. International Journal of Algebra and Computation, 24(02):121-169, 2014.

[Kle99] Bruce Kleiner. The local structure of length spaces with curvature bounded above. Mathematische Zeitschrift, 231(3):409-456, 1999.

[KM09] Jeremy Kahn and Vladimir Markovic. Immersing almost geodesic surfaces in a closed hyperbolic three manifold. Ann. Math., 175:1127-1190, 2009.

[Mal49] A. I. Mal'cev. On a class of homogeneous spaces. Izvestiya Akad. Nauk. SSSR. Ser. Mat., 13:9-32, 1949.

[Man05] Jason Fox Manning. Geometry of pseudocharacters. Geometry and Topology, 9:1147-1185, 2005.

[MM99] Howard A Masur and Yair N Minsky. Geometry of the complex of curves I: Hyperbolicity. Inventiones mathematicae, 138(1):103-149, 1999. 
[MM00] Howard A Masur and Yair N Minsky. Geometry of the complex of curves II: Hierarchical structure. Geometric and Functional Analysis, 10(4):902-974, 2000.

[Mon01] Nicolas Monod. Continuous bounded cohomology of locally compact groups. Number 1758. Springer, 2001.

[MS] P. Mathieu and A. Sisto. Deviation inequalities for random walks. arXiv:1411.7865.

[MT14] Joseph Maher and Giulio Tiozzo. Random walks on weakly hyperbolic groups. arXiv:1410.4173, 2014.

[NR03] G. A. Niblo and L. D. Reeves. Coxeter groups act on CAT(0) cube complexes. J. Group Theory, 6(3):399-413, 2003.

[NS13] Amos Nevo and Michah Sageev. The poisson boundary of cat (0) cube complex groups. Groups, Geometry, and Dynamics, 7(3):653-695, 2013.

[Osi14] D. Osin. Acylindrically hyperbolic groups. Trans. Amer. Math. Soc., to appear, 2014.

[Pan83] Pierre Pansu. Croissance des boules et des géodésiques fermées dans les nilvariétés. Ergodic Theory Dynam. Systems, 3(3):415-445, 1983.

[Pan89] Pierre Pansu. Métriques de Carnot-Carathéodory et quasiisométries des espaces symétriques de rang un. Annals of Mathematics, pages 1-60, 1989.

[Pau01] Scott D. Pauls. The large scale geometry of nilpotent Lie groups. Comm. Anal. Geom., 9(5):951982, 2001.

[Raf07] Kasra Rafi. A combinatorial model for the teichmüller metric. GAFA Geometric And Functional Analysis, 17(3):936-959, 2007.

[RW] Kim Ruane and Stefan Witzel. CAT(0) cubical complexes for graph products of finitely generated abelian groups. arXiv:1310.8646.

[Sag95] Michah Sageev. Ends of group pairs and non-positively curved cube complexes. Proc. London Math. Soc. (3), 71(3):585-617, 1995.

[Sco78] Peter Scott. Subgroups of surface groups are almost geometric. Journal of the London Mathematical Society, 2(3):555-565, 1978.

[Sis13] Alessandro Sisto. Projections and relative hyperbolicity. Enseign. Math. (2), 59(1-2):165-181, 2013.

[SS] L. Sabalka and D. Savchuk. Submanifold projection. ARXIV:1211.3111.

[SW05] Michah Sageev and Daniel T. Wise. The Tits alternative for CAT(0) cubical complexes. Bull. London Math. Soc., 37:706-710, 2005.

[Tay] Samuel J. Taylor. Right-angled Artin groups and Out $\left(F_{n}\right)$ I: quasi-isometric embeddings. Groups, Geometry, and Dynamics. To appear.

[Thu88] William P Thurston. On the geometry and dynamics of diffeomorphisms of surfaces. Bulletin of the American Mathematical Society, 19(2):417-431, 1988.

[Whi49a] J. H. C. Whitehead. Combinatorial homotopy. I. Bull. Amer. Math. Soc., 55:213-245, 1949.

[Whi49b] J. H. C. Whitehead. Combinatorial homotopy. II. Bull. Amer. Math. Soc., 55(5):453-496, 1949.

[Wis] Daniel T. Wise. The structure of groups with a quasiconvex hierarchy. 205 pp. Preprint 2011.

[Wis04] Daniel T. Wise. Cubulating small cancellation groups. GAFA, Geom. Funct. Anal., 14(1):150214, 2004.

[Wis07] Daniel T Wise. Complete square complexes. Commentarii Mathematici Helvetici, 82(4):683-724, 2007.

[Wis11] Daniel T. Wise. From riches to RAAGs: 3-manifolds, right-angled Artin groups, and cubical geometry. In Lecture notes, NSF-CBMS Conference, CUNY Graduate Center, New York, August 2011.

Lehman College and The Graduate Center, CUny, New York, New York, USA

Current address: Barnard College, Columbia University, New York, New York, USA

E-mail address: jason@math.columbia.edu

U. Michigan, Ann Arbor, Michigan, USA

E-mail address: markfhagen@gmail.com

ETH, ZÜrICH, SwitZerland

E-mail address: sisto@math.ethz.ch 